\title{
ENERGY CONSERVATIVE DESIGN AND NONLINEAR CONTROL OF BALANCE IN A HOPPING ROBOT
}

\author{
A Thesis \\ Submitted to the Faculty of Graduate Studies and Research \\ In Partial Fulfillment of the Requirements \\ for the Degree of \\ Master of Applied Science \\ in Electronic Systems Engineering \\ at \\ University of Regina
}

\author{
By \\ Klaus $\mathrm{Nji}$ \\ Ottawa, Ontario
}

July, 2006

(C) Copyright 2006: Klaus $\mathrm{Nji}$ 


$\begin{array}{ll}\begin{array}{l}\text { Library and } \\ \text { Archives Canada }\end{array} & \begin{array}{l}\text { Bibliothèque et } \\ \text { Archives Canada }\end{array} \\ \begin{array}{l}\text { Published Heritage } \\ \text { Branch }\end{array} & \begin{array}{l}\text { Direction du } \\ \text { Patrimoine de l'édition }\end{array} \\ \begin{array}{l}\text { 395 Wellington Street } \\ \text { Ottawa ON K1A ON4 }\end{array} & \begin{array}{l}\text { 395, rue Wellington } \\ \text { Ottawa ON K1A ON4 } \\ \text { Canada }\end{array}\end{array}$

Your file Votre référence ISBN: 978-0-494-20229-6 Our file Notre référence ISBN: 978-0-494-20229-6

NOTICE:

The author has granted a nonexclusive license allowing Library and Archives Canada to reproduce, publish, archive, preserve, conserve, communicate to the public by telecommunication or on the Internet, loan, distribute and sell theses worldwide, for commercial or noncommercial purposes, in microform, paper, electronic and/or any other formats.

The author retains copyright ownership and moral rights in this thesis. Neither the thesis nor substantial extracts from it may be printed or otherwise reproduced without the author's permission.
AVIS:

L'auteur a accordé une licence non exclusive permettant à la Bibliothèque et Archives Canada de reproduire, publier, archiver, sauvegarder, conserver, transmettre au public par télécommunication ou par l'Internet, prêter, distribuer et vendre des thèses partout dans le monde, à des fins commerciales ou autres, sur support microforme, papier, électronique et/ou autres formats.

L'auteur conserve la propriété du droit d'auteur et des droits moraux qui protège cette thèse. $\mathrm{Ni}$ la thèse ni des extraits substantiels de celle-ci ne doivent être imprimés ou autrement reproduits sans son autorisation.
In compliance with the Canadian

Privacy Act some supporting forms may have been removed from this thesis.

While these forms may be included in the document page count, their removal does not represent any loss of content from the thesis.
Conformément à la loi canadienne sur la protection de la vie privée, quelques formulaires secondaires ont été enlevés de cette thèse.

Bien que ces formulaires aient inclus dans la pagination, il n'y aura aucun contenu manquant.

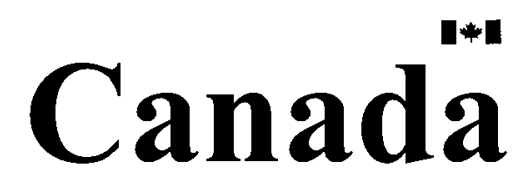




\section{UNIVERSITY OF REGINA}

\section{FACULTY OF GRADUATE STUDIES AND RESEARCH}

\section{SUPERVISORY AND EXAMINING COMMITTEE}

Klaus Nji, candidate for the degree of Master of Applied Science, has presented a thesis titled, Energy Conservative Design and Nonlinear Control of Balance in a Hopping Robot, in an oral examination held on March 24, 2006. The following committee members have found the thesis acceptable in form and content, and that the candidate demonstrated satisfactory knowledge of the subject material.

External Examiner: Dr. John Barden, Faculty of Kinesiology and Health Studies

Supervisor: $\quad$ Dr. Mehran Mehrandezh, Faculty of Engineering

Committee Member: Dr. Luigi Benedicenti, Faculty of Engineering

Committee Member: $\quad$ *Dr. Mohamed El-Darieby, Faculty of Engineering

Chair of Defense: $\quad$ Dr. Nader Mahinpey, Faculty of Engineering

*Unable to attend defense 


\section{Abstract}

The problem of nonlinear identification and energy conservative control of balance in a monopod is addressed in this thesis. A monopod is emulated using a concave balancing mechanism, referred to as the body, mounted on an inverted pendulum, referred to as the leg, via a hip joint. The body curvature, represented by $\phi$, , can be altered and is elected as the design parameter of interest as it is observed that at an optimal body span angle, $\phi_{o p t}$, certain interesting phenomena transpire: The linearized system is transformed from non-minimum to minimum phase, the conditions for feedback linearization of the nonlinear model satisfied, and minimal mechanical power required for stability of the simulated model is observed.

A nonlinear gray-box system identification routine is developed in and implemented, within MATLAB, to estimate certain non-measurable parameters that arise within the original system dynamics. To estimate the optimal angle $\phi_{o p t}$, another non-measurable parameter, the identification routine is further employed for various values of $\phi$. A locus of the transfer function zeros is then used to interpolate the value of $\phi$ at which the system achieves minimum phase behaviour. At this configuration, the magnitude of the transfer function zeros become much larger in comparison with that of the largest pole and therefore has a negligible contribution to the phase characteristics of the system.

After the experimental identification of $\phi_{o p t}$, a Linear Quadratic Regulator with integral action is design and implemented to achieve the control objectives, with the design parameters set as close as physically realizable to the optimal setting. A nonlinear controller based on Feedback Linearization is also designed and implemented to take advantage of one benefit of this proposed design. The performance of the nonlinear controller is also compared 
against that of the LQ Regulator.

Analysis and documentation of the power savings from the proposed design, in relation to existing prototypes, is a subject of further works. Furthermore, the performance of the developed controllers can be further improved via careful tuning to meet more stringent control objectives. 


\section{Acknowledgements}

I would like to thank my supervisor Dr. Mehran Mehrandezh, the pioneer of this novel balancing mechanism, for his constant support during this research. Despite breaking numerous motors and inconsistently attending weekly meetings that conflicted with my shift work schedule, he was always there to provide guidance. Without his constant support, the completion of this project would not have been possible, especially during the final crucial hours.

I would also like to thank the Faculty of Graduate Studies and Research for giving me the opportunity to pursue further studies in control research. Acknowledgement and thanks also goes to the Dr. J. D. Mollard Graduate Award for support issued in the Fall of 2003.

I wish to also thank the following: Kuldip Naik for his help during the software integration struggles; Saeed Poozesh for providing his mechanical expertise. Special thanks goes to Jan Houska from HUMUSOFT s.r.o in the Czech Republic for sending me the pre-released software driver that provided the capability of bidirectional counting within RTWin Target.

I would like to thank CGI Information Systems, my former employer for giving me the iv 
opportunity to earn a living and support my family. The experiences I gained from 5 years of service is helping shape up a positive future for me.

Special thanks goes to my family for their patience and love. Without them I probably would have lost my sanity trying to complete this research while holding a full time job. 


\section{Table of Contents}

Abstract ii

Acknowledgements iv

List of Tables $\quad$ ix

List of Figures $\quad$ x

1 Introduction 3

1.1 Literature Survey on Hopping Robots . . . . . . . . . . . . . . 3

1.2 Contributions to the Literature . . . . . . . . . . . 7

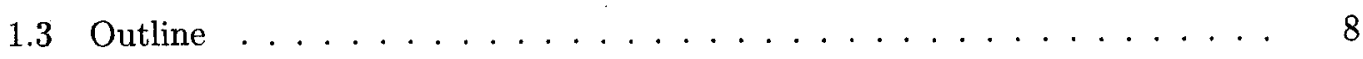

2 Problem Definition and Mathematical Model 10

2.1 Introduction . . . . . . . . . . . . . . . . . . . . 10

2.2 Problem Formulation and Physical Description of System _ . . . . . . 11

2.3 Mathematical Model with Motor Torque as Input . . . . . . . . . . . . . 13

2.4 Mathematical Model with Computed Voltage Signal as Input . . . . . . . 20

2.5 System Equilibrium . . . . . . . . . . . . . . . . . 22

2.6 Open Loop Poles and Zeros of Linearized System . . . . . . . . . . . . 25

2.7 Control Effort Dependency on Span Angle . . . . . . . . . . . . . . 27

2.8 Definition of $\phi$ as Design Parameter . . . . . . . . . . . . 30

3 System Identification 33

vi 
3.1 Introduction . . . . . . . . . . . . . . . . . 33

3.2 Overview of System Identification . . . . . . . . . . . . . . . . . 34

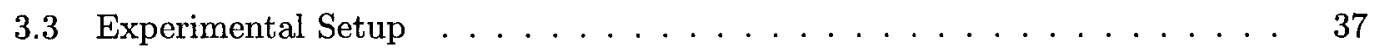

3.4 Nonlinear Gray Box Parametric Identification . . . . . . . . . . . . . . 38

3.5 Experimental Identification of $\phi_{o p t} \ldots \ldots \ldots \ldots \ldots \ldots$

3.6 Sensitivity Analysis . . . . . . . . . . . . . . . . . . . . . . 53

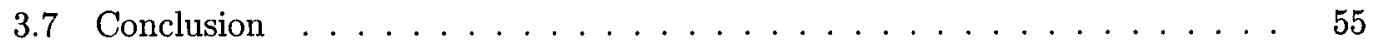

4 Linear Quadratic Regulator (LQR) with Integral Action 56

4.1 Introduction . . . . . . . . . . . . . . . . . . . . 56

4.2 Control Objectives . . . . . . . . . . . . . . . 56

4.3 State Space Description of Acrobot . . . . . . . . . . . . . . 58

4.4 Controller Design . . . . . . . . . . . . . . . . . . . . . . . . 60

4.5 Discretization . . . . . . . . . . . . . . . . . 63

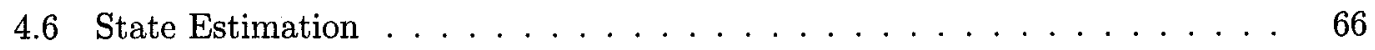

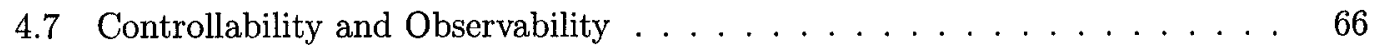

4.8 Simulation Results . . . . . . . . . . . . . . . . . 68

4.8.1 Constant Voltage Disturbance . . . . . . . . . . . . 70

4.8 .2 Periodic Voltage Disturbance . . . . . . . . . . . . . 71

4.8.3 Pulsed Voltage Disturbance . . . . . . . . . . . . . . . 72

4.8.4 Initial Displacement from Equilibrium . . . . . . . . . . 73

4.9 Experimental Results . . . . . . . . . . . . . . . . . . 75

4.10 Mechanical Power vs. Span Angle $\phi \ldots \ldots \ldots \ldots$. . . . . . . . 78

4.11 Conclusion . . . . . . . . . . . . . . . . . . . 79

5 Feedback Linearizing Control $\quad 81$

5.1 Introduction . . . . . . . . . . . . . . . . 81

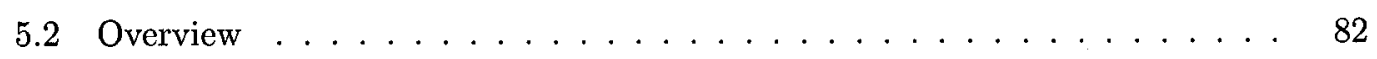

5.3 Controller Design . . . . . . . . . . . . . . . . . 83

5.4 Simulation . . . . . . . . . . . . . . . . . . . . . 89 
5.4 .1 Constant Voltage Disturbance . . . . . . . . . . . . . . . . 91

5.4 .2 Periodic Voltage Disturbance . . . . . . . . . . . . . . . . 91

5.4 .3 Pulsed Voltage Disturbance . . . . . . . . . . . . . . . . 94

5.4 .4 Initial Displacement from Equilibrium . . . . . . . . . . . . . 94

5.5 Experimental Results . . . . . . . . . . . . . . . . . . . . 96

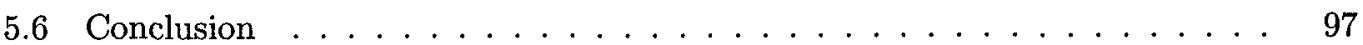

6 Conclusion and Future Works $\quad 99$

6.1 Problems Encountered and Future Works . . . . . . . . . . . . . . 101

6.2 Additional Recommendations . . . . . . . . . . . . . . . . 102

A Operating the 'Rotating Hip' Experimental Setup 104

$\begin{array}{ll}\text { Bibliography } & 108\end{array}$

viii 


\section{List of Tables}

2.1 Description of the System Physical Parameters . . . . . . . . . . . . . . . 14

2.2 PMDC Motor Parameters . . . . . . . . . . . . . . . . 20

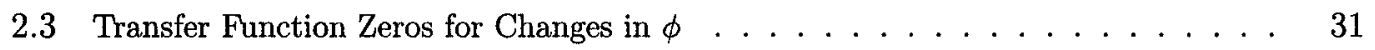

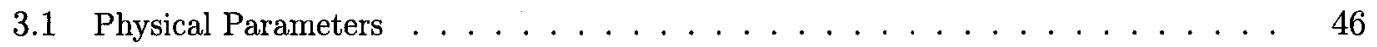

3.2 Estimated Parameters . . . . . . . . . . . . . . . . . . . . 47

3.3 Fit Values Before System Identification . . . . . . . . . . . . . . . 53

3.4 Fit Values After System Identification . . . . . . . . . . . . . . . 53

3.5 Model fit changes for a $40 \%$ parametric uncertainty . . . . . . . . . . . . . 54 


\section{List of Figures}

1.1 Configuration of the Entire Experimental Setup . . . . . . . . . . . . 5

2.1 Physical Structure of the System . . . . . . . . . . . . . . . . . 11

2.2 Ideal Modeling Structure $\ldots \ldots \ldots \ldots \ldots \ldots \ldots$

2.3 Coordinate Frames . . . . . . . . . . . . . . . . . . . . 15

2.4 The Equilibrium Manifold $\ldots \ldots \ldots \ldots \ldots$

2.5 Equilibrium Input Voltage to Actuator . . . . . . . . . . . . . . . . . 24

2.6 Locus of transfer function zeros are $\phi$ is varied from 0 to $90^{\circ} \ldots \ldots 32$

3.1 Overview of System Identification $\ldots \ldots \ldots \ldots \ldots \ldots$

3.2 Physical Configuration of Balancing Mechanism . . . . . . . . . . . 38

3.3 Configuration of the Entire Experimental Setup . . . . . . . . . . . . . 39

3.4 System Used for Identification. . . . . . . . . . . . . . . . . . 43

3.5 Comparison between real and simulation data for $\phi=20^{\circ}$ using raw data, Fit $=$ $-1743 ; 85.03 \ldots \ldots \ldots \ldots \ldots \ldots$

3.6 Comparison between real and simulation data for $\phi=20^{\circ}$ using estimated data, Fit

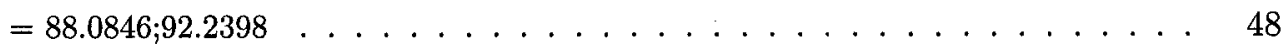

3.7 Comparison between real and simulation data for $\phi=30^{\circ}$ using raw data, Fit $=$ $-1386.829 \ldots \ldots \ldots \ldots \ldots \ldots \ldots \ldots \ldots . \ldots \ldots$ 
3.8 Comparison between real and simulation data for $\phi=30^{\circ}$ using estimated data, Fit $=87.8 ; 98.6 \ldots \ldots \ldots \ldots \ldots \ldots \ldots$

3.9 Comparison between real and simulation data for $\phi=40^{\circ}$ using raw data, Fit $=$ $-979 ; 82.0 \ldots \ldots \ldots \ldots \ldots \ldots$

3.10 Comparison between real and simulation data for $\phi=40^{\circ}$ using estimated data, Fit $=83.3 ; 99.1 \ldots \ldots \ldots \ldots$

3.11 Comparison between real and simulation data for $\phi=50^{\circ}$ using raw data, Fit $=$

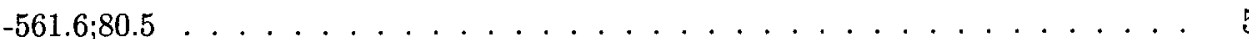

3.12 Comparison between real and simulation data for $\phi=50^{\circ}$ using estimated data, Fit $=82.2 ; 97.8 \ldots \ldots \ldots \ldots \ldots \ldots \ldots \ldots \ldots \ldots \ldots \ldots \ldots$

3.13 Comparison between real and simulation data for $\phi=60^{\circ}$ using raw data, Fit $=$ $-344.4 ; 6.7 \ldots \ldots \ldots \ldots \ldots \ldots \ldots$

3.14 Comparison between real and simulation data for $\phi=60^{\circ}$ using estimated data, Fit $=83.3 ; 98.0 \ldots \ldots \ldots \ldots 2$

3.15 Pole-Zero locus using estimated parameters . . . . . . . . . . . . . . . 54

4.1 The Equilibrium Manifold . . . . . . . . . . . . . . . . 59

4.2 Equilibrium Input Voltage to Actuator . . . . . . . . . . . . . . . . . . . 59

4.3 Typical Block Diagram of a Computer Control System . . . . . . . . . . . . 64

4.4 Simulink Model of Plant and Controller . . . . . . . . . . . . . . . . . . 70

4.5 Simulation Result of LQR Control in Response to a Constant Disturbance . . . . 71

4.6 Simulation Result of LQR Control in Response to a Sinusoidal Disturbance . . . . 72

4.7 Simulation Result of LQR in Response to 2 pulses . . . . . . . . . . . . . . . 73

4.8 Simulation Result of LQR in response to non-zero Initial Conditions . . . . . . . 74

4.9 Open Loop System Response to Sinusoidal Disturbance . . . . . . . . . . . . . . 74

4.10 Experimental Result of LQR in Response to 2 Pulses . . . . . . . . . . . . . . 75 
4.11 Second Experimental Result of LQR in Response to 2 pulses . . . . . . . . . . 76

4.12 Third Experimental Result of LQR in Response to 2 pulses $\ldots \ldots \ldots$

4.13 Real System Subjected to a Single Pulse in Open Loop . . . . . . . . . . . 77

4.14 Mechanical Power vs. $\phi \ldots \ldots \ldots \ldots \ldots$. . . . . . . . . . . . 79

5.1 Simulation Response of FL vs. LQR Control in Response to Constant Disturbance 92

5.2 Simulation Result of FL vs. LQR Control in Response to a Sinusoidal Disturbance 92

5.3 Simulation Result of FL vs. LQR in Response to 2 Pulses . . . . . . . . . . . 93

5.4 Simulation Result of FL vs. LQR Control in response to Non-Zero Initial Conditions 93

5.5 Simulation Result of FL vs. LQR Control in response to Non-Zero Initial Conditions 94

5.6 Simulation Result of FL vs. LQR Control in response to Non-Zero Initial Conditions 95

5.7 Experimental Result of FL control in response to 2 pulses $\ldots \ldots \ldots 9$ 


\section{Nomenclature}

\section{Rigid Body Parameters}

$c_{i} \quad \cos \left(q_{i}\right)$

$c_{i} \quad \sin \left(q_{i}\right)$

$f_{c 20} \quad$ Coulomb friction coefficient at hip joint

$f_{c l 0} \quad$ Coulomb friction coefficient at base joint

$f_{r 1} \quad$ Viscous friction coefficient of around base joint

$f_{r 2} \quad$ Viscous friction coefficient of hip joint

$I_{l 1} \quad$ Moment of Inertia of Link 1 w.r.t its center of gravity

$K_{a m p}$ Linear Gain of Servo amplifier

$l_{1} \quad$ Length of link 1

$l_{2} \quad$ Length of link 2

$L_{c 1} \quad$ Location of gravity of Link 1 w.r.t its center of gravity

$L_{c 2} \quad$ Location of center of gravity of Link 2 w.r.t its rotation axis

$m_{i} \quad$ Arbitrary constants 
$m_{l 1} \quad$ Mass of link 1

$m_{l 2} \quad$ Mass of link 2

\section{Motor Parameters}

$I_{a} \quad$ Armature current of DC motor

$I_{m} \quad$ Inertia of shaft about axis of rotation

$K_{b} \quad$ Back EMF constant

$K_{t} \quad$ Torque Constant

$L_{a} \quad$ Armature inductance

$R_{a} \quad$ Armature resistance

$V_{a} \quad$ Armature voltage of DC motor 


\section{Chapter 1}

\section{Introduction}

Gunter Stein [1] in his classic Hendrick W. Bode lecture during the 1989 IEEE Conference on Decision and Control, once said "Respect the Unstable" for the task of controlling an unstable system is not only a challenging but can be a dangerous undertaking.

\subsection{Literature Survey on Hopping Robots}

Dynamically stable legged robots remain the platform of choice when compared to wheeled and tracked locomotion in terms of versatility, mobility and speed in a continuous and smooth terrain. Legged robots especially capable of stable hopping also can be used as an efficient option for transportation in low gravity environments.

Most of the hopping robots that have been realized in laboratory, especially during the past two decades, have been dynamically stable in the sense that stability is only achievable via a continuous hopping motion. Static stability on the other hand, while a useful property if successfully incorporated with hopping, is a challenge to achieve in such small legged dynamic structures. The low, self-sufficient power requirements of autonomous dynamic machines and the physics of keeping a hopper's center of mass within its small support polygon render the task of achieving static stability a challenging one. 
Nonetheless, the dynamics and balancing of legged systems have been extensively studied over the past two decades, dating back to Matsouka [2], with Raibert making a major contribution to the field. Together with his colleagues, the latter successfully built one-legged, two-legged, and four-legged robots that performed stable hopping, running, centering gaits, and summersaults $[3],[4]$.

The basic structure of Raibert's monopod (i.e. one-legged robot), mainly constructed to understand the dynamics of legged locomotion, comprised of a body mass mounted atop a single leg via a hip joint. The hopper was capable of achieving stable hopping using a simple, yet appropriate, tri-partitioned control scheme. In this scheme, forward velocity was controlled using a foot placement algorithm, hopping height by regulating the system energy stored in a linear spring, and balance by using the body to apply torques onto the hip.

The body in Raibert's planar monopod was constructed with its center of mass above the hip joint. The physics of this setting is synonymous with the well known inverted pendulum [5] and, depending on active link, can be compared in structure to the Acrobot [6], pendubot [7], or even the inverted wedge [8]. The Acrobot and Pendubots, both two link planar robots, are simply variants of the double inverted pendulum, with the fundamental difference between both underactuated systems residing in the location of the lone actuator as illustrated in Figure 1.1 below. On the Acrobot [9], the actuator is located at the base of the active link (the elbow). In contrast, the actuator is situated at the base of the first link (the shoulder) on the Pendubot [10].

The above underactuated mechanical systems have four equilibrium states, although it is more common to find control objectives targeted towards the unstable equilibrium where both links are in the upright position. These systems share several similar and undesirable properties (i.e they are non-minimum phase and inherently unstable systems). 


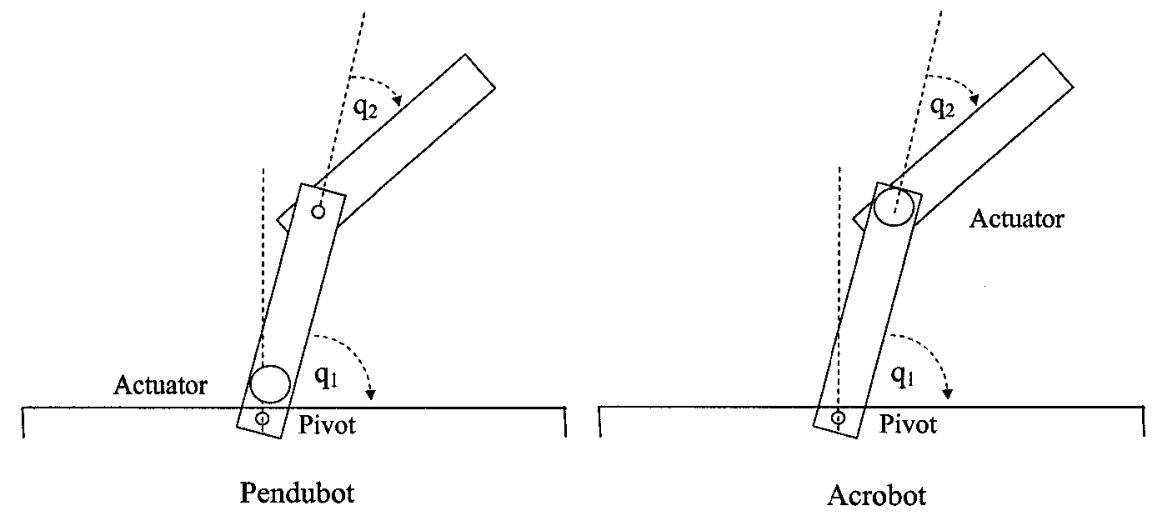

Figure 1.1: Configuration of the Entire Experimental Setup

It is well known that non-minimum phase systems, or systems with transfer function zeros on the right side of the s-plane, are difficult to control due to the high control cost to meet almost any control objective [11]. The reason for the high control cost associated with non-minimum phase systems is the presence of an inverse-response and time-delay within the system dynamics.

Dynamic electromechanical systems are especially prone to the detrimental effects arising from non-minimum phase zeros given that high control costs typically translate to larger and more powerful motors, larger batteries, or larger power sources. The net effect of these larger components is a sluggish and power hungry system, which entirely defeats the purpose of cheap and modern control. When one further considers the Rover deployed to Mars by the Jet Propulsion Laboratory at NASA [12], it immediately becomes evident how an energy conservative strategy is paramount if dynamic machines are to make their place in history.

Raibert already foresaw the setbacks of power hungry systems after using hydraulic 
actuators in his first monopod. He subsequently made attempts to reduce the power demands of the earlier monopods by first replacing the hydraulic actuators with electrical ones [3]. Numerous attempts have been made in pursuit of further reducing the overall power demands of the hopping cycle. Based on the assumption of a loose coupling between the dynamics of height regulation, balance, and forward velocity, as initially proposed by Raibert, the approach has been to make individual amendments on the power demands from each phase of the hopping cycle. For instance, to reduce the power consumption required for balance during stance, efforts have ranged from morphological modifications to the synthesis of more efficient control laws.

For instance, Ringrose [13] built a self-stabilizing running robot with a large circular foot, while Zeglin replaced the linear spring that acted as energy storage in Raibert's model with a bow string in his Bow Legged robot [14]. In the latter, the effect was a noticeable reduction in the leg mass, to about $1 \%$ of body mass, which allowed the hopper to attain higher hopping heights without compromising power savings. Zeglin also introduced passive stability by placing the body's center of mass below the hip joint. Papantoniou [15], Rad et al., [16], and Dummer sand Berkemeier [17] used lighter electric instead of heavy hydraulic actuators. Ahmadi and Buehler added hip and leg compliance, reducing the energy loss during impact at touchdown [18]. McGeer built systems without sensors, actuators or computers [19], which were also very energy efficient, although they had inherent control and intelligent limitations.

From the perspective of more efficient control, especially in light of the recent advances in nonlinear control, analytical studies on simplified system dynamics were performed by Koditscheck and Behler [20] and Vakakis et al. [21]. These studies have subsequently aided in the development of higher order, energy efficient, and robust controllers for each phase in the hopping cycle, which can be assumed to be loosely coupled. Examples of these works 
can be found in [22],[23], [24] and [25], although this list is far from exhaustive.

\subsection{Contributions to the Literature}

The contributions of this work are two fold: A power conserving, inherently stable, balancing mechanism of a monopod is proposed in order to achieve the objective of controlling posture during stance. The proposed structure consists of a concave downward swinging symmetrical body mounted on a single inverted pendulum serving as the leg of a monopod. The balancing structure, also referred to as the body in this thesis, is mounted on the inverted pendulum, also referred to as the leg, at a hip joint. In contrast to some existing balancing prototypes, the center of mass of the structure lies below the hip joint. Furthermore, body movement occurs in a plane below the hip joint. We will show that a specific selection of physical parameters results to a unique body angle, one that is later referred to as the optimal angle $\left(\phi_{o p t}\right)$. At this optimal configuration, the linear approximation of the proposed idealized model is transformed from a non-minimum to a minimum phase system, and the original nonlinear system dynamics is feedback linearizable.

The concept of lowering the body center of mass below the hip, thereby inducing a more stable system, was also used by Gokan et al. in their rope hopping robot [26]. Such structures with the body center of mass below the hip joint and bodily movements occurring predominantly in the plane below the hip can be classified as 'Downward-Swinging' prototypes. Raibert's planar hopper, the hip and leg compliant hopper built by Ahmadi and Buehler, as well as the energy efficient hopper built by Papantoniou, the Acrobot, Pendubots, and double inverted pendulum for instance, where the body center of mass lies above the hip joint and bodily movement occurs predominantly in the plane above the hip joint, can be classified as a 'Upward-Swinging' prototypes. The fundamental difference between the two classes centers around inherent stability of the active link. 'Upward Swinging' 
prototypes have an inherently unstable active link whereas 'Downward Swinging' Prototype have an inherently stable active link.

The second contribution of this work is the synthesis of several control laws that can be further tuned and adapted solely for the purpose of controlling balance in a monopod in the presence of modelling and parametric uncertainty. We are not re-inventing the wheel but using proven formulae to synthesize and test different types of controllers: A Linear Quadratic Regulator (LQR) and Feedback Linearizing controller (EFL) [27]. Integral action is incorporated into the controller solutions in an effort to reduce steady state errors. Experimental results with both controllers within the loop are also presented.

The reason behind the synthesis of two different control laws is to compare the performance of the classic LQR against nonlinear techniques, such as EFL. Given that EFL control is very sensitive to modelling uncertainty, no overwhelming performance improvements are expected of this controller even though successful simulation runs are obtained. For robustness to parametric and modelling uncertainties, other forms of robust control are being considered.

This work gets its motivation from the recent research interest in non-holonomic systems and developments in nonlinear control methodologies. Our inspiration comes from recent projects such as the Mars Rover, the Sony QRIO robot [28], and other advances in Legged and Walking robots. The minimally actuated hopping rover for planetary exploration built by Fiorini, Hayati, Heverly, and Gensler [29] was also a good point of reference.

\subsection{Outline}

The remainder of this thesis is organized as follows: Chapter Two revisits a detailed description of the mechanical structure. In this chapter, the equations governing the dynamics 
of the system are developed and linearized about the unstable or desired equilibrium. Stability analysis of the linearized model is also performed, and an investigation of the power requirements is studied with respect to a certain design parameter.

Chapter Three deals exclusively with system identification. A nonlinear gray box technique is used to estimated certain unmeasurable parameters that appear in the governing dynamics. Exhaustive experimental results obtained from the identification of the optimal design parameter are also presented. System identification is done at numerous system configurations to arrive at the optimal design solution.

Chapter Four deals with the formulation and synthesis of the Linear Quadratic Regulator (LQR) with Integral Action. Simulation and experimental results are presented.

Chapter Five deals with the formulation and synthesis of the Feedback Linearizing Controller with Integral Action. Simulation and a sample experimental result are presented.

We present conclusions and recommendations for future works in Chapter Six. Problems encountered are also outlined in this chapter. 


\section{Chapter 2}

\section{Problem Definition and Mathematical Model}

\section{$2.1 \quad$ Introduction}

In this chapter, an elaborate derivation of the mathematical model for the proposed balancing mechanism of a monopod (i.e. a one-legged robot) is undertaken. First, a detailed physical description of the system is given, followed by a formal definition of the control problem. As in most model-based control exercises, a mechanistic model to predict the dominant system dynamics is formulated with two different types of control inputs. Based on the nature of the control problem, a stability analysis of the linearized system is performed about a desired equilibrium point. A brief analysis of the mechanical power requirement as a function of a certain design parameter concludes the chapter. 


\subsection{Problem Formulation and Physical Description of Sys- tem}

The goal of this project is to design an energy conserving balancing mechanism and control system to maintain balance in a monopod during the stance phase of a hopping cycle. The physical system mainly comprises a concave downward-swinging symmetrical body mounted on an inverted pendulum serving as the non-compliant leg of the monopod. Figure 2.1 presents the real system used in this work. The balancing mechanism, henceforth

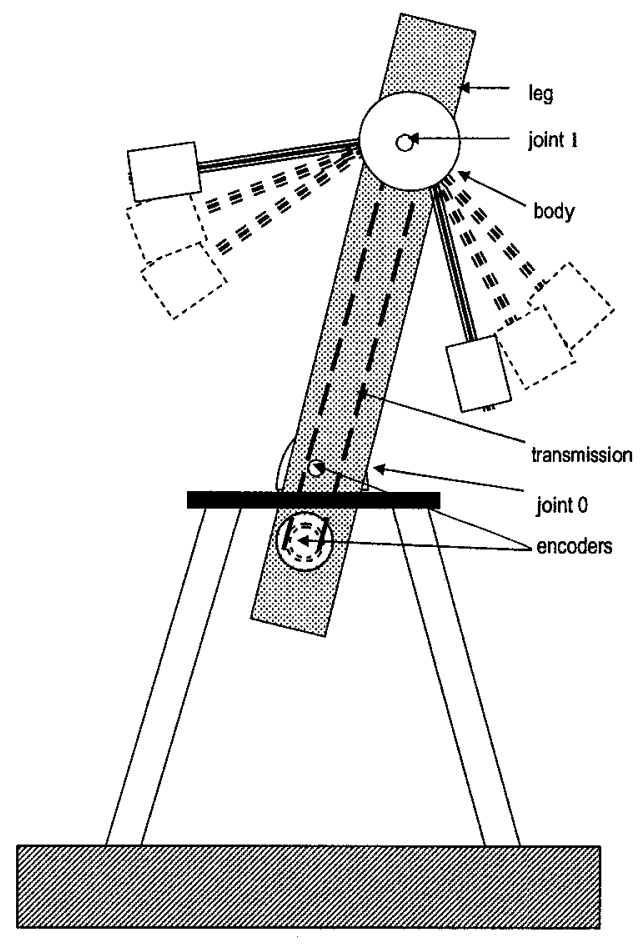

Figure 2.1: Physical Structure of the System

referred to as the body, is mounted on the pendulum, henceforth referred to as the leg, via a joint, henceforth referred to as the hip. The basic structure is similar to a 2-link planar manipulator but for the fact that motion of the active link occurs predominantly in the 
southern hemisphere. The southern hemisphere, in this context, refers to the region below the hip joint.

The body is constructed using two aluminum bars, connected at an angle, $\phi$, at the hip joint. At each end of the aluminum bars is affixed a small mass such that this section of the body can be modelled as concentrated point masses. The links are connected to a platform that allows the selection of ten discrete possible values for $\phi$, which is designated as the design parameter for reasons that will become apparent later. A DC motor attached to the base of the leg provides the torques necessary to induce body motion in response to a disturbance. The motor is mounted at the base of the leg to minimize the adverse torques that would arise from its weight if mounted higher up the leg axis. A chain and sprocket transmission transfers the torque from the motor shaft to the body. Incremental optical encoders are used to measure the angular displacements of both links from a defined equilibrium.

The leg, $80 \mathrm{~cm}$ long and constructed from a $2 \times 4$ " piece of wood, is free to rotate around a pivot, hence, an inverted pendulum by design. This scenario, which mimics a one-legged hopping robot during the stance phase of a hopping cycle, allows one to greatly simplify the hopper's dynamics during stance. The fixed nature of the pivot simulates static friction between the foot of the robot and ground. This friction force is typically assumed to prevent slippage when the monopod is titled during stance.

With the body mounted on the leg via the hip joint, the goal, therefore, is to balance the system around its unstable equilibrium state defined as the point where the leg is vertically up and the body is in its normal stable position. This equilibrium position, defined as a reference in the phase plane, is the only point along the trajectory where encoder readings are set to zero. 


\subsection{Mathematical Model with Motor Torque as Input}

The proposed balancing mechanism consists of a concave downward swinging body modelled as two concentrated masses, $m_{2}$, each located at an equidistance, $l_{2}$, from the hip joint (joint 1). The center of gravity of the body is assumed to be located at a distance, $l_{c 2}$, below the hip joint, as illustrated in Figure 2.2. An armature controlled Permanent Magnet Direct Current (PMDC) motor of mass, $m_{m}$, is affixed to the leg at a distance, $l_{m}$, from the foot (joint 0 ). Motor toque is transmitted to the body via a chain and sprocket assembly.

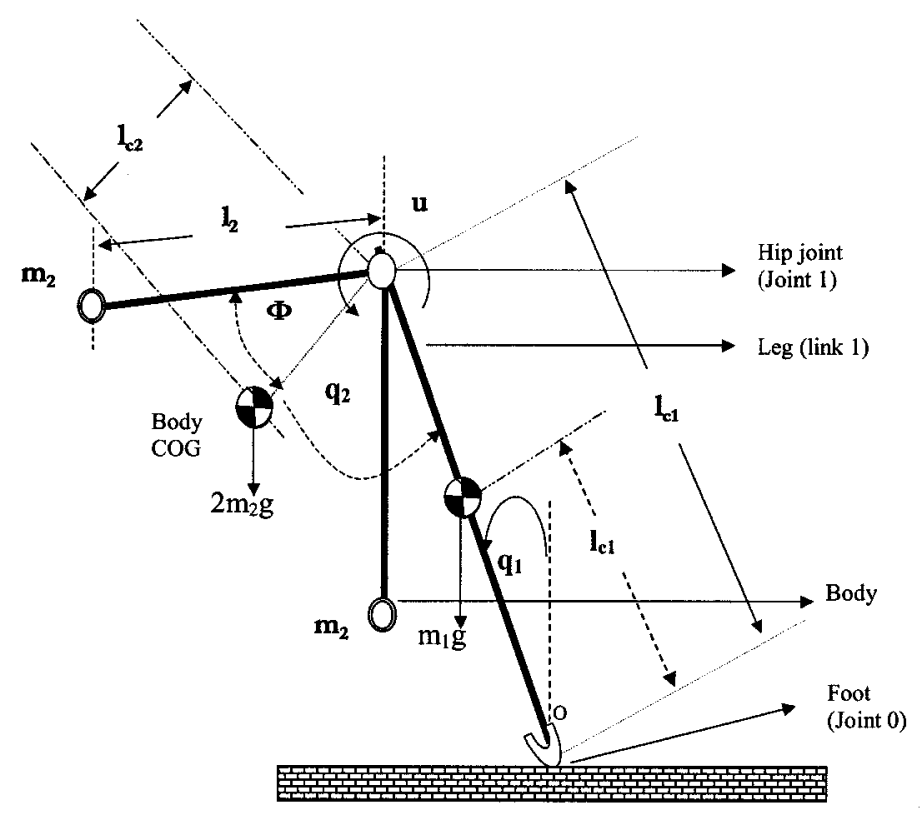

Figure 2.2: Ideal Modeling Structure

The leg is assumed to have a uniformly distributed mass, $m_{l}$, and is of total length $l_{1}$. The center of mass of the leg is located a distance, $l_{c 1}$, from the foot. The moment of inertia of the leg and body about their respective centers of gravity is represented by $I_{L 1}$ and $I_{L 2}$. The mass moment of inertia of the motor's rotor about its axis of rotation along the shaft is denoted by $I_{m}$. Viscous friction coefficients for both joints are labelled $f_{r 1}$ and $f_{r 2}$. Coulomb friction coefficients for both joints are labelled $f_{c 1}$ and $f_{c 2}$. 
Table 2.1: Description of the System Physical Parameters

\begin{tabular}{ll} 
Symbol & Description \\
\hline$m_{1}$ & Mass of leg \\
$m_{2}$ & Mass of body \\
$m_{m}$ & Mass of motor \\
$l_{1}$ & Location of leg COG with respect to foot \\
$l_{2}$ & Location of body COG with respect to hip joint \\
$I_{L 1}$ & Moment of inertia of leg about its COG \\
$I_{L 2}$ & Moment of inertia body about its COG \\
$I_{m}$ & Moment of inertia of motor shaft about its axis of rotation \\
$f_{r 1}$ & Viscous friction coefficient at joint 0 \\
$f_{r 2}$ & Viscous friction coefficient at joint 1 \\
$f_{c 1}$ & Coulomb friction coefficient at joint 0 \\
$f_{c 2}$ & Coulomb friction coefficient at joint 1
\end{tabular}

Half of the angle between the two arms of the body is represented by $\phi$. We will later show, via simulation, that at a specific angle, referred to as $\phi_{\text {opt }}$, the linearized model of the system is transformed from non-minimum to minimum phase, the conditions required for feedback linearization of the nonlinear model are satisfied, and minimal mechanical power required for stability during stance is observed. Table 2.1 summarizes the physical parameters of the system.

In order to describe the position and orientation of a body in space, a coordinate system or frame is typically attached to the object. The position and orientation of this frame is then described with respect to some reference or base frame. In this work, two coordinate frames are used to describe the position and orientation of both links.

The origin of a base coordinate Frame 0 is affixed to the foot and the origin of Frame 1 at the hip joint. Using the Denavit-Hartenberg convention [30,42] axis $z_{0}$ is chosen along the rotational axis of the joint 0 and axis $z_{1}$ along the rotational axis of joint 1 . Axes $y_{0}$ and $y_{1}$ are chosen to represent the vertical axes of both coordinate frames. Axes $x_{0}$ and $x_{1}$ are then chosen to complete the right hand rule. An illustration of the coordinate frame assigned 
to the model is given below. With respect to the above coordinate frames, the angular

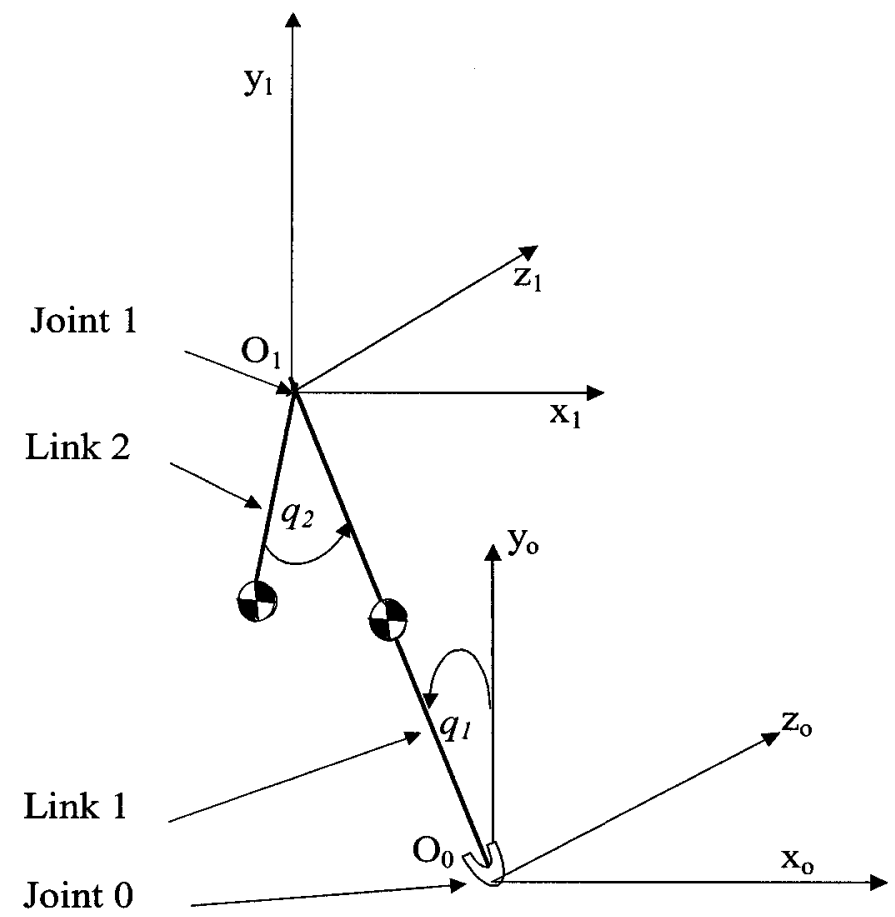

Figure 2.3: Coordinate Frames

displacement of the leg from the vertical $\left(y_{0}\right)$ is represented by $q_{1}$ with counterclockwise rotation denoted as positive. The relative angular displacement from the leg to the body's center of mass is represented by $q_{2}$.

Defining a state vector $q=\left[\begin{array}{ll}q_{1} & q_{2}\end{array}\right]^{T}$, the equations of motion governing any robotic manipulator can be written in the form

$$
\mathbf{M}(\mathbf{q}) \ddot{\mathbf{q}}+\mathbf{C}(\mathbf{q}, \dot{\mathbf{q}}) \dot{\mathbf{q}}+\mathbf{F}_{r} \dot{q}+\mathbf{F}_{c} \operatorname{sgn}(\dot{\mathbf{q}})+\mathbf{G}(\mathbf{q})=\tau
$$

where $M(q)$ represents the inertia matrix and $C(q, \dot{q})$ is a matrix of Coriolis and centrifugal forces. $F_{r}$ is a diagonal matrix of joint frictional constants and $G(q)$ is the gravitational 
matrix.

The governing dynamic equations of the system is obtained using the Langrange formulations given as:

$$
\frac{d}{d t} \frac{\partial \mathcal{L}}{\partial \dot{q}_{i}}-\frac{\partial \mathcal{L}}{\partial q_{i}}=\xi_{i} \quad i=1,2
$$

with the Langrange defined as:

$$
\mathcal{L}=\mathcal{T}-\mathcal{U}
$$

and $T$ and $U$ respectively represent the total kinetic and potential energy of the system. $q_{i}$ represent the generalized coordinates ( $q_{1}$ and $q_{2}$ in our system) and $\xi_{i}$ represents the generalized forces associated with the generalized coordinate $q_{i}$.

However, Sciavicco and Siciliano $[30,140]$ have provided a simpler method of computing the system matrices after defining certain position vectors, which can then be used to obtain the required system Jacobians. Using their method, the inertia matrix can be obtained from the expression

$$
\mathbf{B}(\mathbf{q})=m_{i} J_{P}^{\left(L_{i}\right) T} J_{P}^{\left(L_{i}\right)}+J_{O}^{\left(L_{i}\right) T} I_{L i} J_{O}^{\left(L_{i}\right)}+m_{m i} J_{P}^{\left(m_{i}\right) T} J_{P}^{\left(m_{i}\right)}+J_{O}^{(m i) T} I_{m i} J_{O}^{\left(m_{i}\right) T},
$$

the Coriolis terms from

$$
c_{i j}=\frac{1}{2} \dot{b}_{i j}+\frac{1}{2} \sum_{k=1}^{n}\left(\frac{\partial b_{i k}}{\partial q_{j}}-\frac{\partial b_{j k}}{\partial q_{i}}\right) \dot{q}_{k},
$$

and the gravitational matrix from

$$
g_{i}=\frac{\partial U}{\partial q_{i}}=\sum_{j=1}^{n}\left(m_{i} J_{P i}^{L_{i}}(\mathbf{q})+m_{m i} J_{P i}^{m_{i}}(\mathbf{q})\right)
$$

where $m_{i}$ and $m_{m i}$ respectively represents the mass of each link and associated motor, $p_{i}$ represents the positional vector of the center of gravity of each link with respect to the origin of the base frame, $p_{m i}$, the positional vector of the motor center of mass with respect to the base frame and $g_{0}$, the gravity vector. The expression $b_{i j}$ represents the elements of 
the inertia matrix. $J_{O}$ and $J_{P}$ represent a special set of system matrices computed solely from the position vectors of the links $P_{i}$.

First, we represent the position vector of the origin of the base or reference frame by $\mathbf{P}_{0} . \mathbf{P}_{1}$ and $\mathbf{P}_{L}$, respectively represent the position vectors of frame 1 , and the leg center of mass, with respect to the base frame. The position vector of the body center of mass and that of the motor's center of mass, with respect to the base frame, is represented by $\mathbf{P}_{b}$ and $\mathbf{P}_{m}$, respectively. $\overline{\mathbf{P}}_{b}$ represents the position vector of the body center of mass with respect to frame 1. The above vectors are explicitly given below:

$$
\begin{aligned}
\mathbf{P}_{0} & =\left[\begin{array}{lll}
0 & 0 & 0
\end{array}\right]^{T} \\
\mathbf{P}_{1} & =\left[\begin{array}{lll}
-l_{1} \sin q_{1} & l_{1} \cos q_{1} & 0
\end{array}\right]^{T} \\
\mathbf{P}_{L} & =\left[\begin{array}{lll}
-l_{c 1} \sin q_{1} & l_{c 1} \cos q_{1} & 0
\end{array}\right]^{T} \\
\overline{\mathbf{P}}_{b} & =\left[\begin{array}{lll}
-l_{c 2} \sin \left(q_{1}+q_{2}\right) & -l_{c 2} \cos \left(q_{1}+q_{2}\right) & 0
\end{array}\right]^{T} \\
\mathbf{P}_{b} & =\left[\begin{array}{lll}
\mathbf{P}_{1}+\overline{\mathbf{P}}_{b}
\end{array}\right] \\
\mathbf{P}_{m} & =\left[\begin{array}{lll}
-l_{m} \sin q_{1} & l_{m} \cos q_{1} & 0
\end{array}\right]^{T} \\
\mathbf{Z}_{m} & =\left[\begin{array}{lll}
0 & 0 & 1
\end{array}\right]^{T}
\end{aligned}
$$

For the sake of simplicity, the subscripts $s_{1}, c_{1}, s_{2}, c_{2}, s_{12}, c_{12}$, will be used from this point forward to represent $\sin \left(q_{1}\right), \cos \left(q_{2}\right), \sin \left(q_{2}\right), \cos \left(q_{2}\right), \sin \left(q_{1}+q_{2}\right)$, and $\cos \left(q_{1}+q_{2}\right)$, respectively. Using the above vectors and the chosen coordinate frame, the following Jacobian 
matrices can be computed:

$$
\begin{aligned}
\mathbf{J}_{P}^{(l)}=\left[\begin{array}{cc}
-l_{c 1} c_{1} & 0 \\
-l_{c 1} s_{1} & 0 \\
0 & 0
\end{array}\right] & \mathbf{J}_{P}^{(b)}=\left[\begin{array}{cc}
-l_{1} c_{1}+l_{c 2} c_{12} & l_{c 2} c_{12} \\
-l_{1} s_{1}+l_{c 2} s_{12} & l_{c 2} s_{12} \\
0 & 0
\end{array}\right] \\
\mathbf{J}_{O}^{(l)}=\left[\begin{array}{cc}
0 & 0 \\
0 & 0 \\
1 & 0
\end{array}\right] & \mathbf{J}_{O}^{(b)}=\left[\begin{array}{ll}
0 & 0 \\
0 & 0 \\
1 & 1
\end{array}\right] \\
\mathbf{J}_{P}^{(m)}=\left[\begin{array}{cc}
-l_{m} c_{1} & 0 \\
-l_{m} s_{1} & 0 \\
0 & 0
\end{array}\right] & \mathbf{J}_{O}^{(m)}=\left[\begin{array}{ll}
0 & 0 \\
0 & 0 \\
1 & g_{r}
\end{array}\right]
\end{aligned}
$$

Using the state vector $q=\left[\begin{array}{ll}q_{1} & q_{2}\end{array}\right]^{T}$ and representing the input torque provided by the DC motor by $\tau$, the equations of motion governing the system in the form of Equation 2.3.1 is given by

$$
\begin{aligned}
& {\left[\begin{array}{cc}
m_{2}-2 m_{1} c_{2} & m_{3}-m_{1} c_{2} \\
m_{3}-m_{1} c_{2} & m_{4}
\end{array}\right]\left[\begin{array}{c}
\ddot{q}_{1} \\
\ddot{q_{2}}
\end{array}\right]+\left[\begin{array}{cc}
m_{1} s_{2} x_{4} & m_{1} s_{2}\left(x_{3}+x_{4}\right) \\
-m_{1} s_{2} x_{3} & 0
\end{array}\right]\left[\begin{array}{c}
\dot{q}_{1} \\
\dot{q}_{2}
\end{array}\right]} \\
& +\left[\begin{array}{cc}
f_{r 1} & 0 \\
0 & f_{r 2}
\end{array}\right]\left[\begin{array}{c}
\dot{q}_{1} \\
\dot{q_{2}}
\end{array}\right]+\left[\begin{array}{cc}
f_{c 1} \operatorname{sgn}\left(\dot{q}_{1}\right) & 0 \\
0 & f_{c 2} \operatorname{sgn}\left(\dot{q}_{1}\right)
\end{array}\right]+\left[\begin{array}{c}
-m_{5} s_{1}+m_{6} s_{12} \\
m_{6} s_{12}
\end{array}\right]=\left[\begin{array}{c}
0 \\
\tau_{l}
\end{array}\right],
\end{aligned}
$$

which can be also be expanded to

$$
\begin{array}{r}
\left(m_{2}-2 m_{1} c_{2}\right) \ddot{q}_{1}+\left(m_{3}-m_{1} c_{2}\right) \ddot{q}_{2}+\left(m_{1} s_{2} \dot{q}_{2}+f_{r 1}\right) \dot{q}_{1} \\
+m_{1} s_{2}\left(\dot{q}_{1}+\dot{q}_{2}\right) \dot{q}_{2}+f_{c 1} \operatorname{sgn}\left(\dot{q}_{1}\right)-m_{5} s_{1}+m_{6} s_{12}=0 \\
\left(m_{3}-m_{1} c_{2}\right) \ddot{q}_{1}+m_{4} \ddot{q}_{2}-m_{1} s_{2} \dot{q}_{1}^{2}+f_{r 2} \dot{q}_{2}+f_{c 2} \operatorname{sgn}\left(\dot{q}_{2}\right)+m_{6} s_{12}=\tau_{l}
\end{array}
$$


where

$$
\begin{aligned}
& m_{1}=m_{l 2} l_{1} l_{c 2} \\
& m_{2}=m_{l 1} l_{c 1}^{2}+I_{l 1}+m_{l 2} l_{1}^{2}+m_{l 2} l_{c 2}^{2}+I_{l 2}+m_{m} l_{m}^{2}+I_{m} \\
& m_{3}=m_{l 2} l_{c 2}^{2}+I_{l 2}+I_{m} g_{r} \\
& m_{4}=m_{l 2} l_{c 2}^{2}+I_{l 2}+I_{m} g_{r}^{2} \\
& m_{5}=m_{l 1} g l_{c 1}+m_{l 2} g l_{1}+m_{m} g l_{m} \\
& m_{6}=m_{l 2} g l_{c 2}
\end{aligned}
$$

and a classic friction model, described by Equation 2.3.15, has been used to represent the joint friction [31] as follows:

$$
T_{f}=f_{c i} \operatorname{sgn}\left(\dot{q}_{i}\right)+f_{r i} \dot{q}_{i}
$$

for $\mathrm{i}=1$ and 2 , where

$$
\operatorname{sgn}(\dot{q})= \begin{cases}+1 & \dot{q}>0 \\ 0 & \dot{q}=0 \\ -1 & \dot{q}<0\end{cases}
$$

with $f_{c i}$ representing the Coulomb friction and $f_{r i}$ representing the viscous friction as described earlier in this section.

For the sake of control purposes, it is desirable to express the system input in terms of the supply voltage to the servo amplifier driving the DC motor. In the proceeding section, the dynamics of the motor is presented and subsequently integrated into the above system equations such that the generalized input to the system is the input voltage, $u$ to a servo amplifier. 
Table 2.2: PMDC Motor Parameters

\begin{tabular}{ll} 
Parameter & Description \\
\hline$i$ & Armature Current \\
$L_{a}$ & Armature Inductance \\
$R_{a}$ & Armature Resistance \\
$k_{m}$ & Motor torque constant \\
$u$ & Computed control signal \\
$k_{a}$ & Servo amplifier gain in voltage mode \\
$\tau_{m}$ & Torque generated by rotor \\
$\theta_{m}$ & Angular displacement of motor shaft.
\end{tabular}

\subsection{Mathematical Model with Computed Voltage Signal as Input}

The dynamics of a PMDC motor is governed by two coupled equations [32]:

$$
\begin{aligned}
L_{a} \frac{d i_{a}(t)}{d t}+R_{a} i_{a}(t)+k_{b} \dot{\theta}_{m}(t) & =k_{a} u(t) \\
\tau_{m}(t) & =k_{m} i_{a}(t)
\end{aligned}
$$

where the variables are defined in Table 2.2 .

Considering the motor driving the load though a gearing mechanism with ratio $g_{r}$, gearing efficiency $\eta$, and ignoring the inertia of the gears, the torque balance for the entire actuator assembly is

$$
\tau_{m}(t)=I_{m} \ddot{\theta}_{m}(t)+B_{m} \dot{\theta}_{m}(t)+T_{f} \operatorname{sgn}\left(\dot{\theta}_{m}\right)+\frac{\tau_{l}(t)}{\eta g_{r}}
$$


where

$$
\begin{aligned}
\tau_{m} & =\text { Torque at the motor input shaft } \\
\tau_{l} & =\text { Torque experienced by load } \\
B_{m} & =\text { Motor internal friction constant } \\
T_{f} & =\text { Motor internal friction torque }
\end{aligned}
$$

Assuming that the armature inductance, $L_{a}$, is relatively smaller than other terms, its effect can be neglected. Assuming $\theta_{m}=g_{r} q_{2}$ and combining (2.4.1), (2.4.2), and (2.4.3) results ins

$$
T_{l}(t)=\frac{\eta g_{r} k_{a m p} k_{m}}{R_{a}} u(t)-\eta g_{r}^{2}\left(\frac{k_{b} k_{m}}{R_{a}}+B_{m}\right) \dot{q}_{2}(t)-\eta g_{r} T_{f} \operatorname{sgn}\left(\dot{q}_{2}\right)-\eta g_{r}^{2} I_{m} \ddot{q}_{2}(t) .
$$

Combining the second order differential equations in (2.3.11) with (2.4.4) results in

$$
\begin{array}{r}
\left(m_{2}-2 m_{1} c_{2}\right) \ddot{q}_{1}+\left(m_{3}-m_{1} c_{2}\right) \ddot{q}_{2}+\left(m_{1} s_{2} \dot{q}_{2}+f_{r 1}\right) \dot{q}_{1} \\
+m_{1} s_{2}\left(\dot{q}_{1}+\dot{q}_{2}\right) \dot{q}_{2}+f_{c 1} \operatorname{sgn}\left(\dot{q}_{1}\right)-m_{5} s_{1}+m_{6} s_{12}=0 \\
\left(m_{11}-m_{12} c_{2}\right) \ddot{q}_{1}+m_{13} \ddot{q}_{2}-m_{12} s_{2} \dot{q}_{1}^{2}+m_{14} \dot{q}_{2}+m_{15} \operatorname{sgn}\left(\dot{q}_{2}\right)+m_{16} s_{12}=u
\end{array}
$$


where

$$
\begin{aligned}
m_{7} & =\frac{\eta g_{r} k_{a m p} k_{m}}{R_{a}} \\
m_{8} & =\eta g_{r}^{2}\left(\frac{k_{b} k_{m}}{R_{a}}+B_{m}\right) \\
m_{9} & =\eta g_{r}^{2} I_{m} \\
m_{10} & =\eta T_{f} g_{r} \\
m_{11} & =\frac{m_{3}}{m_{7}} \\
m_{12} & =\frac{m_{1}}{m_{7}} \\
m_{13} & =\frac{m_{4}+m_{9}}{m_{7}} \\
m_{14} & =\frac{f_{r 2}+m_{8}}{m_{7}} \\
m_{15} & =\frac{f_{c 2}+\eta g_{r} T_{f}}{m_{7}} \\
m_{16} & =\frac{m_{6}}{m_{7}}
\end{aligned}
$$

and $u$ is the required computed control voltage. Equations (2.4.5) and (2.4.6) will be linearized and used for stability analysis and controller synthesis.

\subsection{System Equilibrium}

The first step in analyzing any nonlinear system is to linearize it about some nominal operating point. Linearization allows the control engineer to gather local behavior of the system about the nominal equilibrium. Before linearizing the system, however, the nominal operation point, which is typically a system equilibrium point, must be appropriately defined.

An equilibrium point ${ }^{1}$ is a state in which a system would remain if it were unperturbed by external disturbances [33]. Thus, at the equilibrium point, system velocities are zero

\footnotetext{
${ }^{1}$ In the phase plane, an equilibrium point is a singular point
} 
(i.e.

$$
\dot{q}_{1}=\dot{q}_{2}=\ddot{q}_{1}=\ddot{q}_{2}=0
$$

) such that

$$
\begin{aligned}
& f_{1}\left(q_{1}, q_{2}\right)=0 \\
& f_{2}\left(q_{1}, q_{2}\right)=0
\end{aligned}
$$

where $f_{1}$ and $f_{2}$ represent the equations in (2.4.5) and (2.4.6), respectively. The values of the equilibrium points can be achieved by solving (2.5.2) and (2.5.3) simultaneously.

Performing the mathematics and substituting (2.5.1) into (2.5.2) and (2.5.3) results in the equilibrium equations:

$$
\begin{aligned}
m_{6} \sin \left(q_{10}+q_{20}\right)-m_{5} \sin \left(q_{10}\right) & =0 \\
m_{16} \sin \left(q_{1}+q_{20}\right)+m_{15} & =u_{e q}
\end{aligned}
$$

where $q_{10}$ and $q_{20}$ define the equilibrium points of $q_{1}$ and $q_{2}$, respectively. Equation (2.5.4) defines a continuous equilibrium manifold around which the system can be regulated while (2.5.5) defines the motor input voltage that is needed to maintain the equilibrium point. Thus, for a given value of $q_{10}$, there exists a corresponding value of $q_{20}$ such that an equilibrium manifold equation is satisfied i.e.

$$
x_{20}=-x_{10}+\sin ^{-1}\left(\frac{m_{5} \sin \left(x_{10}\right)}{m_{6}}\right) .
$$

A plot of the equilibrium points given by (2.5.6) is given in Figure 2.4 with clockwise angular displacement represented by a negative sign. A plot of the input voltage required to maintain each equilibrium position is given by Figure 2.5. Note that these plots, based on unmeasurable system parameters computed using first principles or from simple experiments, will be repeated after the unmeasurable parameters have been estimated in Chapter 3. Amongst the equilibrium set is the singular or equilibrium point 


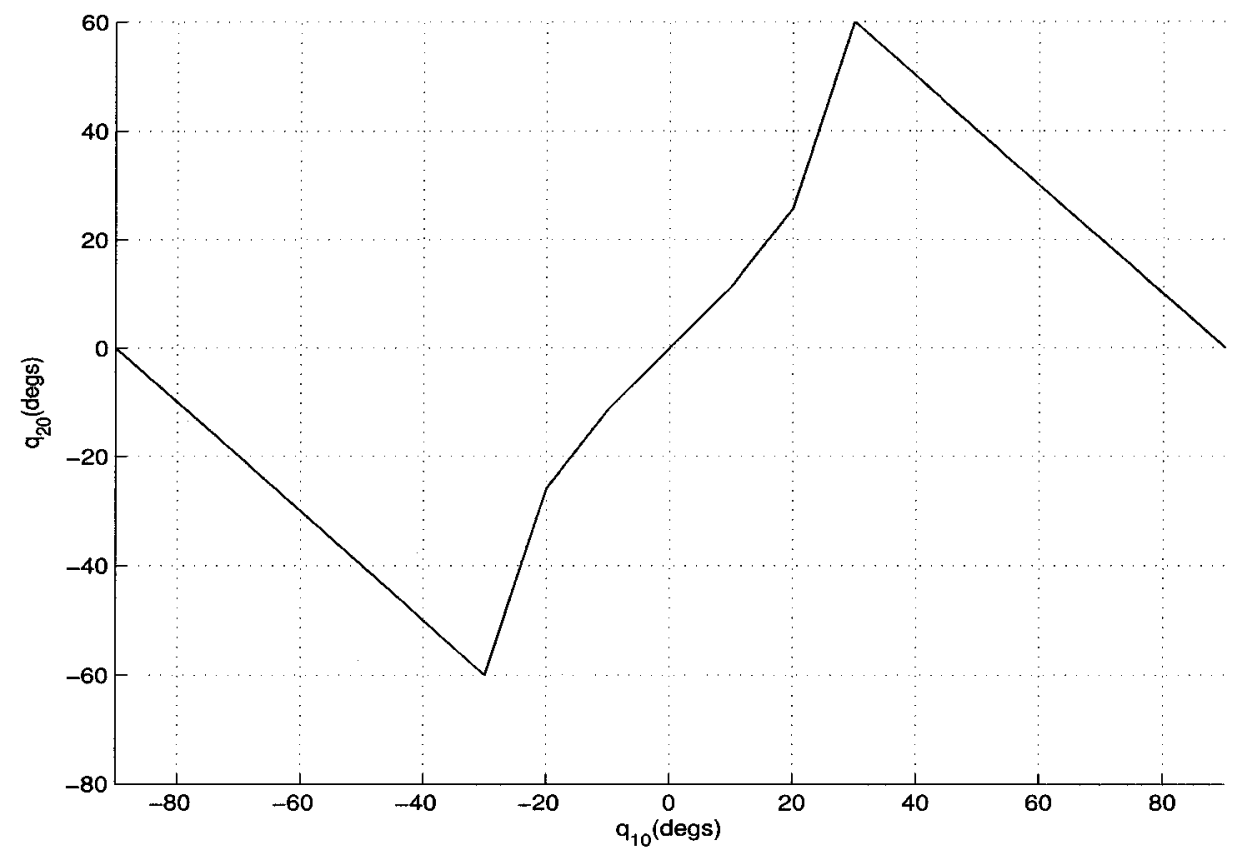

Figure 2.4: The Equilibrium Manifold

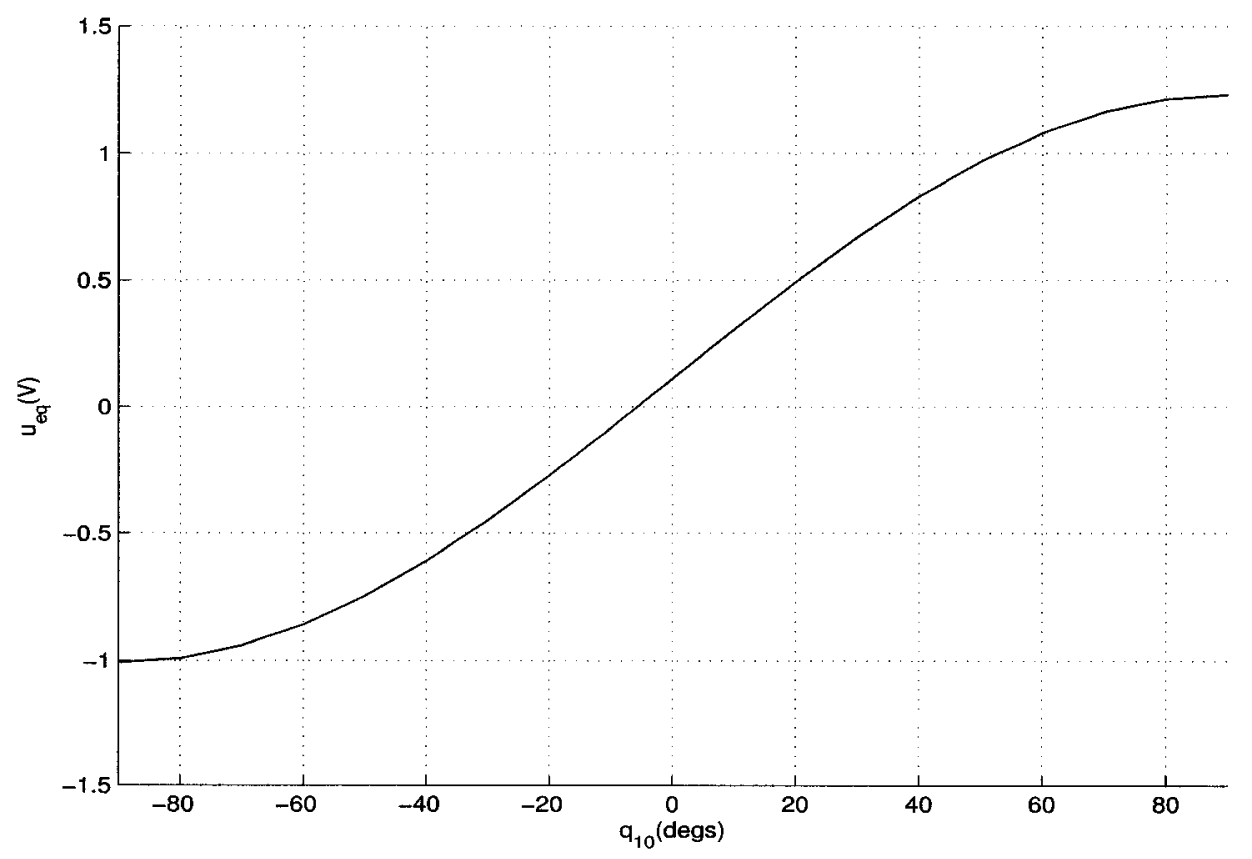

Figure 2.5: Equilibrium Input Voltage to Actuator 


$$
\begin{aligned}
& q_{10}=0 \\
& q_{20}=0 \\
& u_{e q} \approx 0,
\end{aligned}
$$

which is the only point of interest. The point $\left(q_{10}=0, q_{20}=0, u_{e q} \approx 0\right)$ is selected as the nominal operating point ${ }^{2}$ about which the original nonlinear dynamics, given by (2.4.5) and (2.4.6), will be linearized and system stability analysis performed. Worthy of note is a non-zero input voltage required to maintain the system about the nominated nominal equilibrium. This voltage, though less than the motor deadband of $0.3 \mathrm{~V}$, is a function of both the Coulomb friction manifested by the leg joint (joint 1) as well as the internal friction torque of the motor. In order words, if the motor has to overcome a larger overall 'dry' friction torque within a close neighborhood of the nominal equilibrium, the value of the equilibrium voltage will be larger as well.

The equilibrium manifold described by (2.5.4) and illustrated in Figure 2.4 describes a one to one mapping between the output variable of interest $q_{1}$ and the actuated degree of freedom (DOF) $q_{2}$. Therefore, a control system, during the process of driving the output $q_{1}$ to $q_{10}=0$ as defined by the control objectives, will inadvertently drive the state variable $q_{2}$ to $q_{20}=0$. The system can, therefore, be considered as a single-input-single output (SISO) system allowing one to apply the pole-zero concept in SISO linear theory to analyze system stability.

\subsection{Open Loop Poles and Zeros of Linearized System}

One of the objectives of this exercise is to design a balancing mechanism that can be used to maintain an upright posture in a monopod with conservative energy demands. This translates to stabilization of the plant about the singular points defined in an (2.5.7) for

\footnotetext{
${ }^{2}$ In accordance with the control objectives
} 
the the rotational angles of each link, as described in the previous section.

Before engaging in controller synthesis, a stability analysis of the open loop plant around the desired equilibrium will be performed to allow for a better understanding and definition of the control objective. Furthermore, this analysis would later provide an insight into the selection of a design parameter- a parameter which influences the overall mechanical power consumption of the system.

A linear model of the system is first obtained by linearizing the original dynamics given in 2.4.5 about the unstable equilibrium position with the states and control input set to zero, (i.e. $q_{1}=q_{2}=\dot{q}_{1}=\dot{q}_{2}=u=0$ ). Defining the state matrix $\mathbf{x}=\left[\begin{array}{llll}q_{1} & q_{2} & \dot{q}_{1} & \dot{q}_{2}\end{array}\right]^{3}$ the linearized dynamics about $\mathbf{x}=\left[\begin{array}{llll}0 & 0 & 0 & 0\end{array}\right]$ is given as

$$
\begin{array}{r}
\dot{\mathbf{x}}=\mathbf{A}_{\mathbf{o}} \mathbf{x}+\mathbf{B}_{\mathbf{o}} u \\
\mathbf{y}=\mathbf{C}_{\mathbf{o}} \mathbf{x}+D_{o} u
\end{array}
$$

where

$\mathbf{A}_{o}=\left[\begin{array}{cccc}0 & 0 & 1 & 0 \\ 0 & 0 & 0 & 1 \\ \frac{m_{16}\left(m_{3}-m_{1}\right)+m_{13}\left(m_{5}-m_{6}\right)}{\Delta} & \frac{m_{16}\left(m_{3}-m_{1}\right)-m_{13} m_{6}}{\Delta} & \frac{-m_{1} 3 f_{r 1}}{\Delta} & \frac{m_{14}\left(m_{3}-m_{1}\right)}{\Delta} \\ \frac{\left(m_{5}+m_{6}\right)\left(m_{12}-m_{11}\right)+m_{16}\left(2 m_{1}-m_{2}\right)}{\Delta} & \frac{m_{6}\left(m_{11}-m_{12}\right)+m_{16}\left(2 m_{1}-m_{2}\right)}{\Delta} & \frac{f_{r 1}\left(m_{11}-m_{12}\right.}{\Delta} & \frac{-m_{14}\left(m_{2}-2 m_{1}\right)}{\Delta}\end{array}\right]$

$$
\begin{aligned}
\mathbf{B}_{o} & =\left[\begin{array}{c}
0 \\
0 \\
\frac{-m_{3}+m_{1}}{\Delta} \\
\frac{m_{2}-2 m_{1}}{\Delta}
\end{array}\right] \quad \mathbf{C}_{o}=\left[\begin{array}{llll}
1 & 0 & 0 & 0
\end{array}\right] \quad D_{o}=0 \\
\Delta & =\left(m_{12}--m_{11}\right)\left(m_{3}-m_{1}\right)+m_{13}\left(m_{2}-2 m_{1}\right)
\end{aligned}
$$

and the constants $m_{i}, i=1: 16$ are given in (2.3.13) and (2.4.7).

\footnotetext{
${ }^{3}$ Throughout this thesis, the variables $q_{i}$ and $x_{i}, i=1,2$ will be used interchangeably
} 
The open loop transfer function relating the output of interest, $q_{1}$, to the control input $u$ is given by

$$
P(s)=\frac{q_{1}(s)}{u(s)}=\frac{B_{o}(3) s^{2}+B_{o}(4) A_{o}(3,2)-A_{o}(4,2) B_{o}(3)}{s^{4}-\left(A_{o}(4,2)+A_{o}(3,1)\right) s^{2}+\operatorname{det}\left(A_{o}\right)}
$$

where the coefficients of the laplace operator in the transfer function are elements of the system triple $\left(A_{o}, B_{o}, C_{o}\right)$ given in (2.6.3) and (2.6.4). It can be easily verified that the zeros of the above transfer function are given by the expression

$$
p_{o}= \pm \sqrt{\frac{m_{6}}{m_{1}-m_{3}}}, \quad m_{1} \neq m_{3} .
$$

A brief discussion on the effects of the location of open loop zeros on control effort follows. The idea is to allow an appreciation of the energy conservative nature of the novel balancing mechanism and choice in design parameters.

Before venturing further, it should be noted that the linearized system has one unstable open loop pole, implying an unstable open loop system as expected. The purpose of the control system will be to drive the unstable pole to the stable region of the s-plane. Controllers, on the other hand, cannot influence the location of transfer function zeros. Hence, any attempt to minimize the detrimental effect of unstable transfer function zeros simply by manipulating the physical design will be beneficial to the system. We will see why in the next section.

\subsection{Control Effort Dependency on Span Angle}

One of the main contributions of this work is the design of a balancing mechanism for a monopod that consumes less mechanical power as compared to existing prototypes. To aid in the selection of a suitable set of design parameters, a qualitative analysis of the mechanical power requirements is first performed simply by observing the location of the 
open loop transfer function zeros. Before doing so, it is worth reviewing the concepts of internal stability of Linear Time Invariant (LTI) Systems. Even though the original system is nonlinear, it is assumed that system operation occurs predominantly within a close neighborhood to the origin such that the dominant behavior of the system can be represented by the corresponding LTI set of equations in (2.6.1) and (2.6.2).

A LTI system whose transfer function has strict left half plane (LHP) zeros possesses a stable internal dynamics. Such systems are referred to as a minimum phase (MP) system.

Definition 2.7.1. The internal dynamics of a system is the part of the dynamics that is not observable from the external input-output relationship [34].

A non-minimum phase (NMP) system, on the other hand, has transfer function zeros on the right half plane (RHP) or an associated time delay within the system dynamics. Such systems are also characterized by unstable internal dynamics, which represents undesirable phenomena, 'outside' the input-output relationship, such as violent vibrations of mechanical members. NMP systems are difficult to control because their step response is typically characterized by undershoot. NMP systems also suffer from the Waterbed Effect, meaning that small sensitivity to noise can be achieved over one frequency range only at the expense of large sensitivity in another frequency range [35]. Moreover, the application of certain control schemes such as Input-Output Linearization (IOL), a variant of feedback linearization, is possible only if the internal dynamics of the system is stable.

Transfer function zeros, however, are intrinsic properties of linear systems. Slotine and Li [27] extended the concept of zeros to nonlinear systems in the form of the zero-dynamics.

Definition 2.7.2. Zero dynamics is defined as the internal dynamics of the system when the system output is kept at zero by the input [36].

For linear systems, the roots of the zero dynamics are exactly the zeros of the system 
transfer function. Thus linear systems with strict LHP zeros poses stable internal dynamics, permitting control effort to be constrained within conservative bounds.

For nonlinear systems, on the other hand, the relationship is not very clear, even though it can be shown that local asymptotic stability of the zero dynamics is enough to guarantee local asymptotic stability of the internal dynamics [37]. Stability of the zero dynamics of a nonlinear system can be determined using the Lyaponuv's Linearization Stability theorem, which states:

Theorem 2.7.1. - If the linearized system is strictly stable (i.e if all eigenvalues of the system matrix, $A_{o}$, are strictly in the left half of the s-plane), then the equilibrium point is asymptotically stable for the actual nonlinear system.

- If the linearized system is unstable (i.e at least one of the eigenvalues of the system matrix, $A_{o}$, is strictly in the right half of the s-plane), then the equilibrium point is unstable for the actual nonlinear system.

- If the linearized system is strictly marginally stable (i.e if all eigenvalues of the system matrix, $A_{o}$, are strictly in the left half of the s-plane but atleast one of them lies on the $j \omega$ axis ), then one cannot conclude anything from the linear approximation, as the equilibrium point may be stable, asymptotically stable, or unstable for the nonlinear system.

The zero-dynamics corresponding to the linearized model is simply the linearization of the zero dynamics of the nonlinear model. From the Lyaponuv's Linearization theorem, one can ,therefore, conclude that local stability of the zero dynamics for the nonlinear model can also be extracted from the location of the transfer function zeros. Thus, if a linear system is NMP, the nonlinear system can also be considered NMP. The same applies for minimum phase linear systems. 


\subsection{Definition of $\phi$ as Design Parameter}

Refering to (2.6.7), it can be seen that the system can be made minimum or non-minimum phase simply by altering the location of the transfer function zeros. The location of these transfer function zeros is governed by the denominator of $(2.6 .7)$ given that $\left(m_{6}>0\right)$, which in turn is a function of the angle between the two arms of the body represented by $\phi$. Consider, for a moment, the case in which $m_{1}=m_{3}$ such that the transfer function zeros are of very large magnitude or nonexistence. Making the simplifying assumption

$$
I_{L 2}=m_{2}\left(l_{2}^{2}-l_{c 2}^{2}\right)
$$

for the moment of inertia of the body and solving from $\phi$ from the expression $m_{1}=m_{3}$ using the definitions in $(2.3 .10)$, results in

$$
\phi=\phi_{o p t}=\cos ^{-1}\left(\frac{m_{2} l_{2}^{2}+I_{m} g_{r}}{m_{2} l_{1} l_{2}}\right) .
$$

This specific value of $\phi$ is referred to as $\phi_{o p t}$ for reasons that will become apparent towards the end of this chapter. First, we will consider the changes in the location of the open loop transfer function zeros as a function of $\phi$.

Within the range $0 \leq \phi<\phi_{\text {opt }}$ the transfer function 2.6.6 has one undesirable RHP zero for $m_{3}<m_{1}$. The zeros lie very close to the origin when $\phi=0$ and traverse the s-plane, along the real axis towards $\pm \infty$ as $\phi$ approaches $\phi_{o p t}$. As such, the linear system is always non-minimum phase for $\phi<\phi_{\text {opt }}$.

Numerous reports have been presented on the detrimental effect of NMP zeros based on the Bode Integral [38]. Francis and Zames [39] qualitatively showed that requiring the magnitude of the sensitivity function, $|S(j \omega)|$, to be arbitrarily small over some frequency interval forces it to be large elsewhere. $|S(j \omega)|$ directly quantifies such feedback properties as output disturbance rejection and robustness to small parameter variation, while the magnitude of complementary sensitivity function $|T(j \omega)|=|1-S(j \omega)|$ quantifies the response 
of the feedback system to sensor noise. Freudenberg and Looze illustrated that large values of $|S(j \omega)|$ reduce the close loop gain margin [40] and further showed that the presence of RHP zeros forces the system to exhibit undershoot to a step response. In summary, NMP zeros are not desirable, especially when low power consumption is a design concern.

Within the range $\phi_{\text {opt }}<\phi \leq 90^{\circ}$ the transfer function given by Equation (2.6.6) has two complex conjugate zeros along the $j \omega$ axis for $m_{3}>m_{1}$. Within this range, the system is no longer NMP and could be considered marginally minimum phase (MMP). Starting at very large magnitudes when $\phi=\phi_{\text {opt }}$, the zeros move towards the origin along the imaginary axis as $\phi$ approaches $90^{\circ}$. As such, the linear system is always MMP for $90^{\circ} \geq \phi>\phi_{\text {opt }}$.

Goodwin, Woodyatt, Middleton and Shim [41], studied the effect of zeros in the $j \omega$ axis and concluded that a system with $j \omega$ zeros of sufficiently small magnitude relative to the poles will always exhibit overshoot to a step response. They further demonstrated that the presence of an upper limit on the permissible overshoot of the output signal places an effective lower bound on the achievable settling time of the closed loop system when the plant has zeros on or near the imaginary axis. This therefore implies that systems with $j \omega$ axis zeros of sufficiently large magnitude neither suffer from the aforementioned shortcoming nor the detrimental effect of NMP zeros. Being able to directly influence the phase characteristics of the system by simply varying one variable is the main reason behind the choice of $\phi$ as the design parameter. Table 2.3 lists the exact zero location as $\phi$ is varied from 0 to $60^{\circ}$. Figure 2.6 further illustrates the trajectory of the transfer

Table 2.3: Transfer Function Zeros for Changes in $\phi$

\begin{tabular}{|l|c|c|c|c|c|c|c|}
\hline$\phi$ & $0^{\circ}$ & $10^{\circ}$ & $20^{\circ}$ & $20^{\circ}$ & $40^{\circ}$ & $50^{\circ}$ & $60^{\circ}$ \\
\hline zeros & \pm 15.7581 & \pm 17.1810 & \pm 26.9791 & $\pm 20.6659 i$ & $\pm 10.8636 i$ & $\pm 7.3409 i$ & $\pm 5.2506 i$ \\
\hline
\end{tabular}

function zeros as $\phi$ is varied from $0^{\circ}$ to $60^{\circ}$. Starting from $\phi=0$, the transfer function zeros traverse the real axis to a very large value when $\phi=\phi_{\text {opt }}$. From Figure, $\phi_{o} p t$ appears to lie 


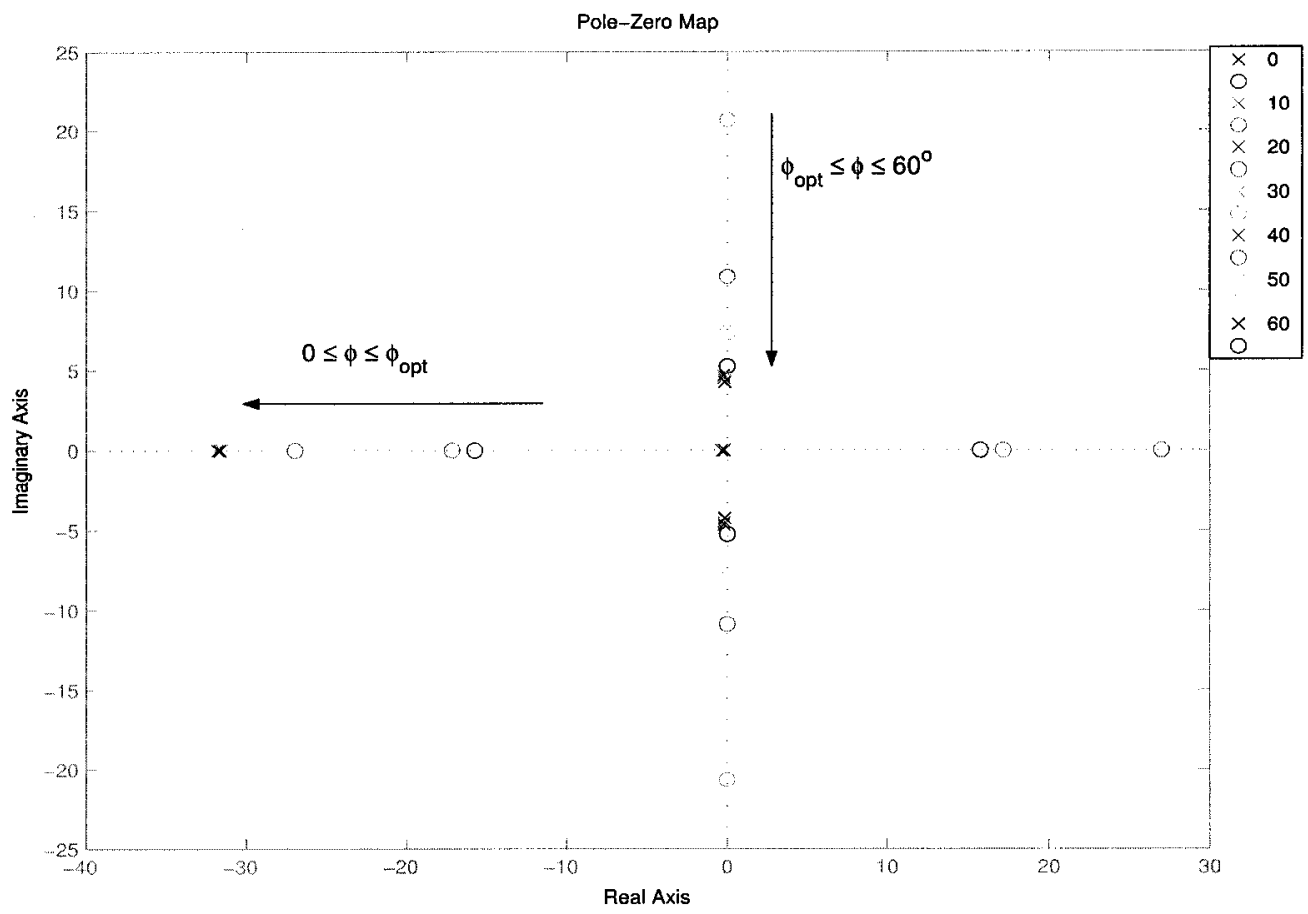

Figure 2.6: Locus of transfer function zeros are $\phi$ is varied from 0 to $90^{\circ}$

between $20^{\circ}$ and $30^{\circ}$. For values of $\phi>\phi_{\text {opt }}$, these transfer function zeros traverse to the imaginary axis and move closer to the origin as $\phi$ is increased further. In the next chapter, an experimental value of $\phi_{\text {opt }}$ will be obtained via system identification. In Chapter 4, a linear control law will be synthesized and the effect of changes in $\phi$ on the control effort required to maintain stability will be analyzed. 


\section{Chapter 3}

\section{System Identification}

A mathematical representation of a physical phenomenon is the very basis of model-based control design. The method of obtaining this mathematical representation or model depends on the process complexity and the amount of a priori information available on the process dynamics. System identification is a statistical method of obtaining the mathematical model of a process entirely from data or estimating certain meaningful physical parameters that cannot be accurately measured, yet exist in a mathematical model that has been computed from first principles.

\subsection{Introduction}

The mathematical modelling of a physical process or phenomenon is very useful in many disciplines. Mathematical models allow one to gain a better understanding of process dynamics via simulation. If one considers the case of an expensive plant operating in a dangerous regime; manned aircraft, chemical processes, nuclear reactors and space robotics readily fo example, mathematical models provide a low cost option that allows the control engineer to gain an insight on controller performance once within the loop, thus saving development cost. Models can also be used in optimization problems and to predict events, such as 
weather patterns, stock prices, interest rates and much more.

All real processes such as discontinuity, hysteresis, static friction, jump phenomena and many more are nonlinear, however, and their dynamics cannot be exactly described using mathematical relationships. Even when the dominant behavior of the process can be well captured using well known mathematical formulae, there often are certain physical parameters that are not measurable and can only be estimated using field data. For instance, the dynamics of a robotic manipulator can be accurately modelled using kinematic relationships between the links [42], [30]. Nonetheless, rigid body parameters, such as the moment of inertia and the center of gravity of each link, are difficult to measure and are typically estimated from input-output data in the process of system identification.

\subsection{Overview of System Identification}

System Identification is the method by which a process model is obtained using input-output data and non-measurable parameters within a pre-computed mathematical model are estimated. The models used in the identification procedure are classified as White Box, Gray Box, or Black Box, depending on how much prior knowledge is available on the process [43, 15]. Irrespective of the nature of the model being used, the goal of the identification procedure is to obtain a mathematical representation that is capable of capturing the dominant dynamics of the real process as the overall quality of the resulting mathematical representation places an upper bound on the performance of the final problem solution. For instance, the performance of a model-based controller will depend on how well the model captures the essential dynamics of the process it represents.

Figure 2.1 briefly illustrates the concept of system identification. Here, the model output $\hat{y}$ is compared against that of the real process, $y$, corrupted with the noise vector $n$. 
The objective of the identification procedure is to obtain a model, $f$, that minimizes the difference between the process and model output, $e$, according to some performance criterion. Before engaging in the identification of the plant parameters used in this thesis, it

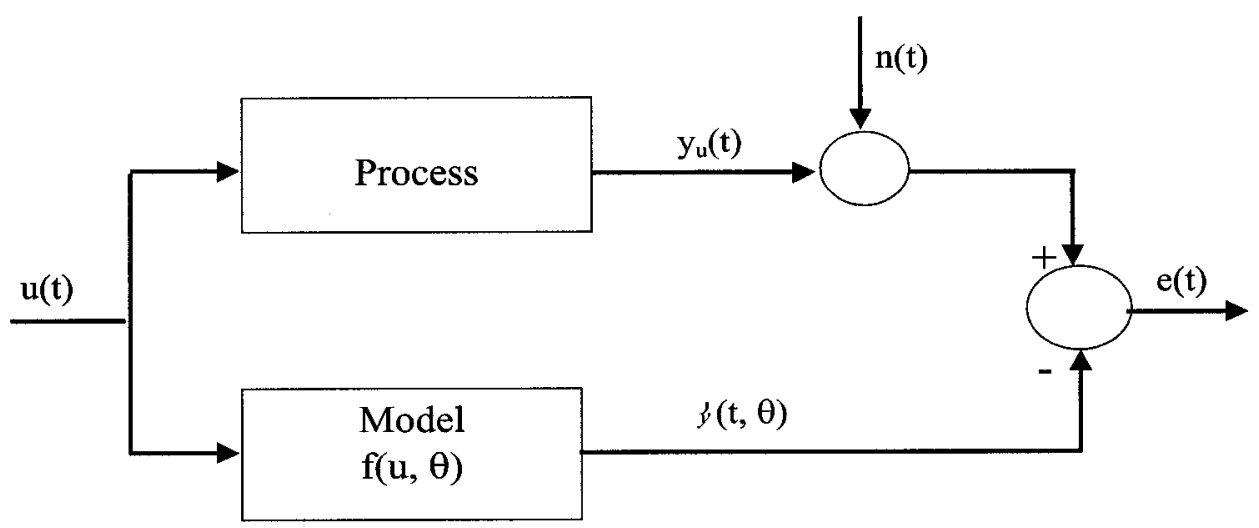

Figure 3.1: Overview of System Identification

is worthwhile reviewing five major steps that make up the system identification process.

1. Selection of an Input Channel: In the case of multiple input systems, this step involves the selection of inputs that will be used to excite the behaviors of interest. In the case of single input systems, such as the one used in this thesis, the choice is obvious.

2. Selecting an excitation signal: The excitation signal is of utmost importance, as it presents the only opportunity to excite the dynamics of the interest in the real process.

3. Selecting a model architecture: This is one of the most difficult steps, given that there can exist numerous models that can adequately describe any given process. The final decision is often based on the problem type or intended use of the model. For instance, a decent mathematical model is sufficient for control purposes. 


\section{Selecting a set of model parameters and estimation of the parameter}

values: Once a model architecture has been selected from the previous step, the choice of model parameters is a relatively straight forward task. Depending on how the parameters appear in the model structure, they can be estimated using linear or nonlinear optimization techniques. For model architectures derived from empirical formulae (white box), the parameters typically represent meaningful physical quantities. In most robotic applications, these parameters typically include the moment of inertia of links and friction coefficients between joints.

Linear optimization techniques are often used to exploit the linearity property of a manipulator model with respect to a suitable set of dynamic parameters [30, 143]. In the case of underactuated manipulator models, a case in point is the model used in this thesis, obtaining the set of dynamic parameters is not a straight forward proposition; hence, a nonlinear optimization technique is employed, as will be explained later in this chapter.

5. Validating the model: After the parameters have been determined from the previous step, the integrity of the model is validated using fresh data. This will allow the engineer to predict the performance of the overall problem solution based on the fit between the real and simulated process output.

The above paragraphs briefly describe the system identification procedure. For a more comprehensive coverage of the subject, the reader can refer to either of the following sources [43] and [44].

As mentioned earlier, the selection of a model architecture and excitation signal are the most crucial steps in system identification. This becomes evident when one considers the following scenario: In Black Box identification, where no prior information is available on the process and the model parameters have no physical interpretation, the burden of extracting any important process behavior lies entirely on the choice of the excitation signal. 
Black box models are used when a process cannot be accurately described using empiricalmathematical relationships.

In Gray box identification, however, some prior knowledge about the system is available. As previously mentioned, there still are certain parameters in the model that cannot be measured and will have to be estimated using input-output data. Estimation of these parameters is often guided by some prior knowledge, such as an allowable operational range or the type of parametric value.

While prior knowledge facilitates the selection of a model architecture, it does not make the selection of an excitation signal an easier task. Careful thought has to be put into the selection of an excitation signal such that its frequency content excites only the process dynamics of interest and not high frequency un-modelled dynamics commonly found in mechanical systems. Before dwelling further on this, the experimental setup for this project will now be introduced.

\subsection{Experimental Setup}

The objective of this thesis is to develop a balancing mechanism and control system that can maintain an upright posture using minimal mechanical power in a $2 \mathrm{DOF}$, one legged robot. The balancing mechanism or body is inherently stable and constructed such that it lowers the overall center of gravity of the host system. The body is made up of two links with masses concentrated at each end. The links are attached to the hip joint and are separated by angle $\phi$. The hip joint is constructed such that $\phi$ can be varied, allowing one to study how this design parameter influences the stability properties and mechanical power requirements of the system. An image of the body is given in Figure 3.2 below. The balancing mechanism is mounted on a 2" $\mathrm{x} 4$ " piece of wood $80 \mathrm{~cm}$ long. This serves as the non-compliant leg in a 


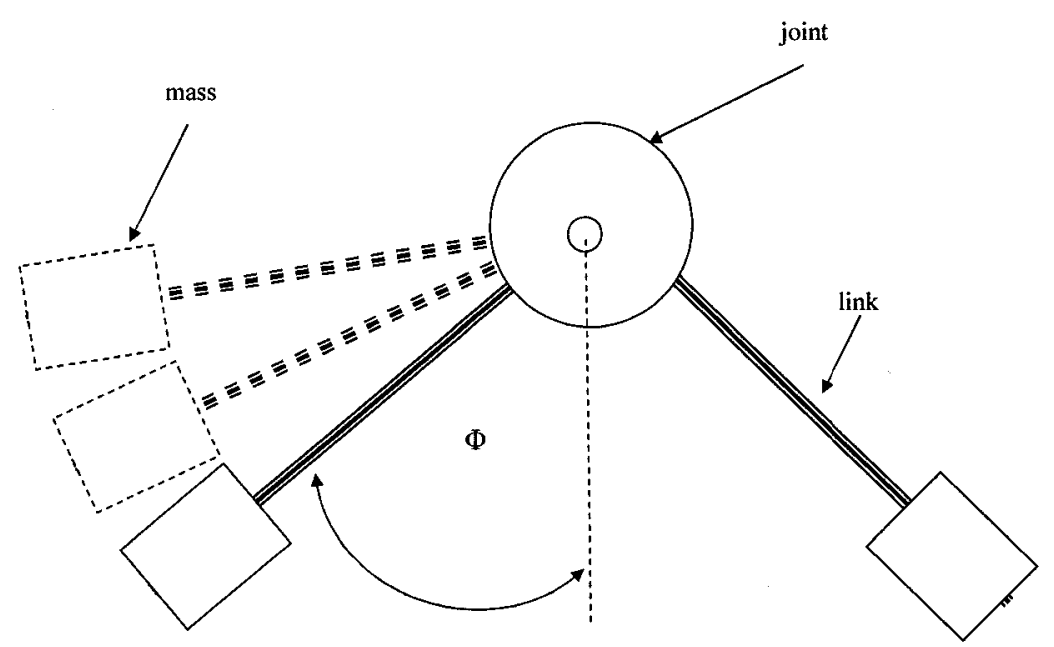

Figure 3.2: Physical Configuration of Balancing Mechanism

hopping robot during stance. At the base of the leg is mounted a Permanent Magnet DC motor powered by an Advanced Motion servo amplifier [45]. The specifications of the motor and servo amplifier are listed later in this chapter. A chain and sprocket assembly is used to transmit the power from the motor to the balancing mechanism. Control signals generated in the MATLAB/Simulink [46] environment are communicated to the hardware using the Mathworks Real Time Windows Target via a National Instruments Data Acquisition card (NIDAQ 6036E) [47]. The rotational angles of both links are measured using incremental optical encoders with a resolution of 2500 and 1000 PPR. The entire mechanical system is mounted on a stand as shown in Figure 3.3.

\subsection{Nonlinear Gray Box Parametric Identification}

System Identification is the method by which a process model is obtained using inputoutput data and non-measurable parameters within a pre-computed mathematical model are estimated. One of the most difficult tasks in the system identification procedure is the selection of a model structure. This task is facilitated if the physical phenomenon can be 


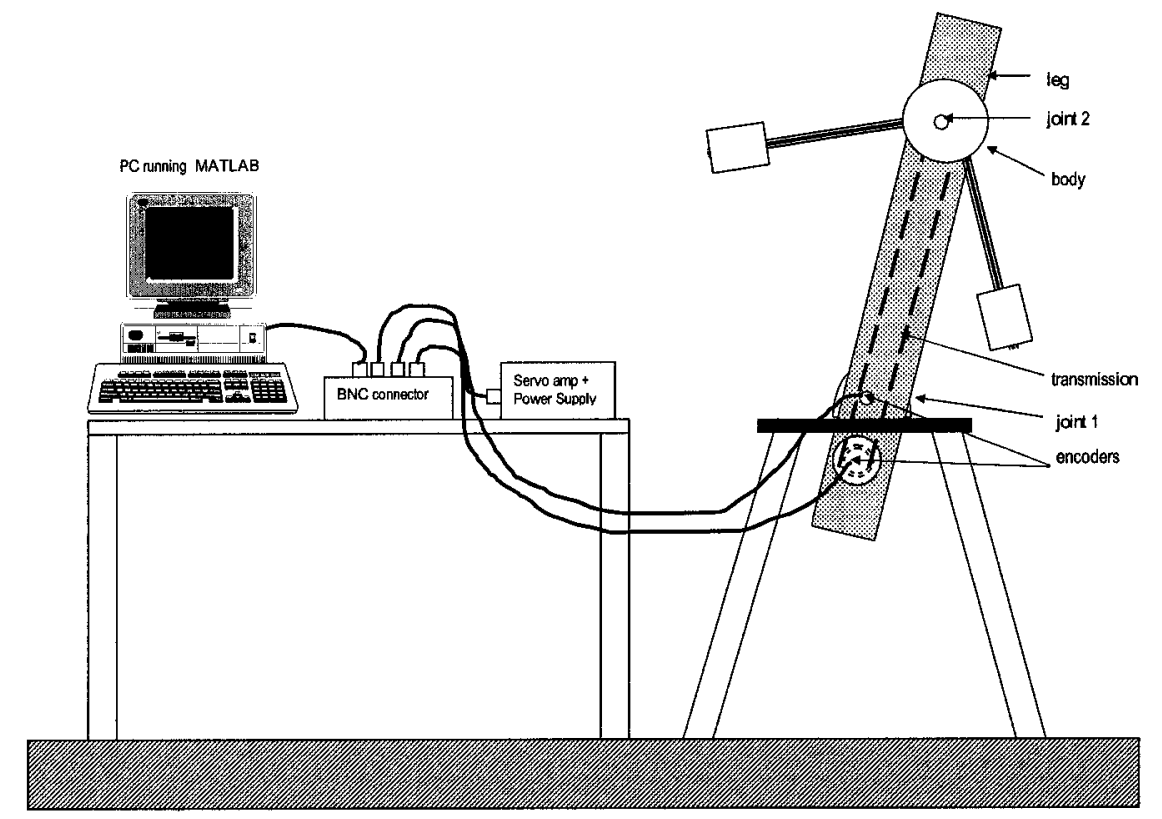

Figure 3.3: Configuration of the Entire Experimental Setup

well described using first principles related to the dynamics of the electromechanical system. An identification procedure based on a model structure, obtained using principles, which is a nonlinear function of the unknown parameters, is referred to as Nonlinear Gray Box Identification $[43,15]$.

The starting point in Nonlinear Gray-box identification is the determination of a continuous time nonlinear state space model structure of the form

$$
\begin{aligned}
& \dot{x}=f(t, x(t), \theta, u(t)) \\
& y=h(t, x(t), \theta, u(t), w(t))
\end{aligned}
$$

where $f$ and $g$ are nonlinear functions, $x(t)$ the state vector, $y(t)$ the output vector, and $\theta$, the unknown parameter vector to be estimated. $u(t)$ represents the excitation signal, and $w(t)$, the measurement disturbance signal, assumed to be white for simplicity, and $t$ denotes the time vector.

As mentioned earlier, the objective of the identification procedure is to find the parameter vector, $\theta$, that minimizes a cost function according to a certain optimization criterion. 
A commonly used optimization criterion is the minimization of a cost function based on the squared error between the real process output $y(t)$ and the estimated process output $\hat{y}(t, \theta)$. This cost function is expressed mathematically as

$$
V_{N}=\frac{1}{N} \sum_{i=1}^{N} e^{2}(t, \theta)
$$

for a given input-output data, where $e$ which represents the difference between the process output, $y$, and predicted output, $\hat{y}$, for our purposes will be explicitly given by

$$
e(t, \theta)=y(t)-\hat{y}(t, \theta)
$$

The parameter vector $\theta$ is estimated by applying a prediction error method, which performs a numerical optimization of the criterion 3.4.3 using an iterative numerical search algorithm. Such algorithms require an initial estimate of the parameter vector $\theta_{\boldsymbol{o}}$, and the quality of the final solution will depend on the choice of initial estimate.

First, we select a model structure from which the parameter vector to be estimated can be extrapolated. While the goal of this work is to stabilize the system about its upright unstable equilibrium, system identification is performed around the stable downward equilibrium for safety and stability reasons. It is assumed that the estimated parameters equally apply around both equilibriums.

The model structure chosen is the mathematical model of the robot around its stable downward equilibrium, as shown in Figure 3.4. In the state space form, the dynamics around the stable equilibrium is given as

$$
\left[\begin{array}{c}
\ddot{q}_{1} \\
\ddot{q}_{2}
\end{array}\right]=\left[\begin{array}{l}
f_{3}\left(q_{1}, q_{2}, \dot{q}_{1}, \dot{q}_{2}\right)+g_{3}\left(q_{2}\right) u \\
f_{4}\left(q_{1}, q_{2}, \dot{q}_{1}, \dot{q}_{2}\right)+g_{4}\left(q_{2}\right) u
\end{array}\right]
$$


where

$$
f_{3}=\frac{\left(\begin{array}{l}
\left(-m_{3} m_{12} s_{2} \dot{q}_{1}^{2}-m_{3} m_{14} \dot{q}_{2}-m_{1} c_{2} m_{12} s_{2} \dot{q}_{1}^{2}-m_{1} c_{2} m_{14} \dot{q}_{2}+m_{13} \dot{q}_{1} f_{r 1}\right. \\
-2 m_{13} \dot{q}_{1} m_{1} s_{2} \dot{q}_{2}-m_{13} m_{1} s_{2} \dot{q}_{2}^{2}-\frac{m_{3}\left(f_{c 2}+m_{10}\right) \operatorname{sgn}\left(\dot{q}_{2}\right)}{m_{7}}+m_{13} m_{5} s_{1}+m_{13} m_{6} s_{12} \\
\left.-\frac{m_{1} c_{2}\left(f_{c 2}+m_{10}\right) \operatorname{sgn}\left(\dot{q}_{2}\right)}{m_{7}}-m_{3} m_{16} s_{12}-m_{1} c_{2} m_{16} s_{12}+m_{13}\left(f_{c 1}\right) \operatorname{sgn}\left(\dot{q}_{1}\right)\right)
\end{array}\right)}{\left(m_{11} m_{3}+m_{11} m_{1} c_{2}+m_{12} c_{2} m_{3}+m_{12} c_{2}\right)^{2} m_{1}-m_{13} m_{2}-2 m_{13} m_{1} c_{2}}
$$

$f_{4}=\frac{\left(\begin{array}{l}\left(-\left(-m_{1} s_{1} \dot{q}_{2}+f r 1\right) \dot{q}_{1}+m_{1} s_{2}\left(\dot{q}_{1}+\dot{q}_{2}\right) \dot{q}_{2}-m_{5} s_{1}-m_{6} s_{12}-\frac{f_{c 1} \operatorname{sgn}\left(\dot{q}_{1}\right)}{m_{3}+m_{1} c_{2}}\right. \\ -\left(m_{2}+2 m_{1} c_{2}\right)\left(-m_{3} m_{12} s_{2} \dot{q}_{1}^{2}-m_{3} m_{14} \dot{q}_{2}-m_{1} c_{2} m_{12} s_{2} \dot{q}_{1}^{2}-m_{1} c_{2} m_{14} \dot{q}_{2}\right. \\ -2 m_{13} \dot{q}_{1} m_{1} s_{2} \dot{q}_{2}+m_{13} \dot{q}_{1} f_{r 1}-m_{13} m_{1} s_{2} \dot{q}_{2}^{2}-\frac{m_{3}\left(f_{c 2}+m_{10}\right) s g n\left(\dot{q}_{2}\right)}{m_{7}}+m_{13} m_{5} s_{1} \\ \left.\left.-m_{3} m_{16} s_{12}-\frac{m_{1} c_{2}\left(f_{c 2}+m 10\right) \operatorname{sgn}\left(\dot{q}_{2}\right)}{m_{7}}-m_{1} c_{2} m_{16} s_{12}+m_{13} m_{6} s_{12}+m_{13} f_{c 1} \operatorname{sgn}\left(\dot{q}_{1}\right)\right)\right)\end{array}\right)}{\left(m_{11} m_{3}+m_{11} m_{1} c_{2}+m_{12} c_{2} m_{3}+m_{12} c_{2}\right)^{2} m_{1}-m_{13} m_{2}-2 m_{13} m_{1} c_{2}}$

$$
\begin{aligned}
g_{3} & =\frac{m_{3}+m_{1} c_{2}}{\left(m_{11} m_{3}+m_{11} m_{1} c_{2}+m_{12} c_{2} m_{3}+m_{12} c_{2}\right)^{2} m_{1}-m_{13} m_{2}-2 m_{13} m_{1} c_{2}} \\
g_{4} & =\frac{-m_{2}-2 m_{1} c_{2}}{\left(m_{11} m_{3}+m_{11} m_{1} c_{2}+m_{12} c_{2} m_{3}+m_{12} c_{2}\right)^{2} m_{1}-m_{13} m_{2}-2 m_{13} m_{1} c_{2}}
\end{aligned}
$$


and the constants, $m_{i}, i \in[1: 16]$ are given again as follows:

$$
\begin{aligned}
m_{1} & =m_{l 2} l_{1} l_{c 2} \\
m_{2} & =m_{l 1} l_{c 1}^{2}+I_{l 1}+m_{l 2} l_{1}^{2}+m_{l 2} l_{c 2}^{2}+I_{l 2}+m_{m} l_{m}^{2}+I_{m} \\
m_{3} & =m_{l 2} l_{c 2}^{2}+I_{l 2}+I_{m} g_{r} \\
m_{4} & =m_{l 2} l_{c 2}^{2}+I_{l 2}+I_{m} g_{r}^{2} \\
m_{5} & =m_{l 1} g l_{c 1}+m_{l 2} g l_{1}+m_{m} g l_{m} \\
m_{6} & =m_{l 2} g l_{c 2} \\
m_{7} & =\frac{\eta g_{r} k_{a m p} k_{m}}{R_{a}} \\
m_{8} & =\eta g_{r}^{2}\left(\frac{k_{b} k_{m}}{R_{a}}+B_{m}\right) \\
m_{9} & =\eta g_{r}^{2} I_{m} \\
m_{10} & =\eta T_{f} g_{r} \\
m_{11} & =\frac{m_{3}}{m_{7}} \\
m_{12} & =\frac{m_{1}}{m_{7}} \\
m_{13} & =\frac{m_{4}+m_{9}}{m_{7}} \\
m_{14} & =\frac{f_{r 2}+m_{8}}{m_{7}} \\
m_{15} & =\frac{f_{c 2}+\eta g_{r} T_{f}}{m_{7}} \\
m_{16} & =\frac{m_{6}}{m_{7}}
\end{aligned}
$$

The unmeasurable quantities in the above system constants make up the parameter vector $\theta$ given as

$$
\theta=\left[\begin{array}{llllllll}
L_{c 1} & L_{c 2} & I_{L 1} & I_{L 2} & f_{r 1} & f_{r 2} & f_{c 1} & f_{c 2}
\end{array}\right]^{T}
$$

where all variables were defined in Section 2.3.

Estimation of the parameter $\theta$ is done in two steps. In the first step two initial estimates, $\theta_{01}$ and $\theta_{02}$, are obtained using simple experiments and empirical formulae. In the 


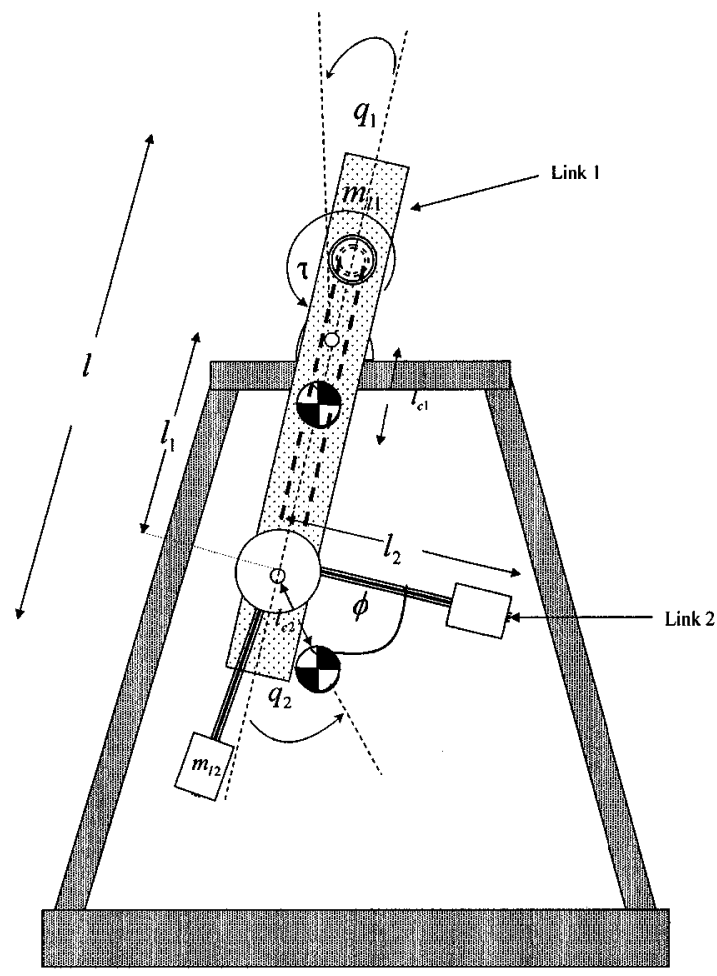

Figure 3.4: System Used for Identification.

second step, each initial estimate is passed to an optimization routine which searches for a parameter vector $\theta$ that minimizes the sum of squares of the error between the real and simulated output state variables at every time instant. The initial estimate that returns the lowest residual is selected.

A chirp signal is used to excite the system for the generation of input-output data. The advantages of a chirp signal are persistent excitation and continuity in terms of the amplitude levels and frequency content [48]. A chirp signal is also chosen as it delivers a waveform with a crest factor of $\sqrt{2}$ not too far above the theoretical recommended value of 1 [49]. The crest factor defines the amount of input power delivered by the signal and is defined as the ratio of the peak amplitude of the signal to the effective root mean square (rms) value, where effective means that only the signal power in the frequency range of interest is used in the rms calculation. For instance, signals with an impulsive behavior 
have a large crest factor and will, for any given peak value, inject much less power into the system than a signal with a lower crest factor.

A frequency sweep from of $0.5-3 \mathrm{~Hz}$ is selected, as this is sufficient to excite the dominant dynamics of the electromechanical system. Frequency ranges above $3 \mathrm{~Hz}$ tend to excite high frequency un-modelled dynamics, such as slackness in the drive train.

The selection of a sampling rate is coupled to the systems' dominant time constant, which also defines the bandwidth. Sampling at rates substantially greater than system bandwidth may lead to data redundancy, numerical issues, and modelling of high frequency artifacts likely due to noise. Sampling at rates lower than system dynamics leads to difficulties determining the correct system parameters as well as the problems introduced by aliasing. A common rule of thumb is to sample signals at 10 times the bandwidth of the system (or bandwidth of interest) for the model [50]. Assuming uncertainty exists in the system bandwidth, and provided that a very fast data acquisition environment is available, it is useful to sample as fast as possible and then use digital pre-filtering and decimation to reduce the sampling rate to the desired value.

Based on the above guidelines, a sampling rate, $T_{s}$, of $0.05 \mathrm{~s}$ is chosen for the identification procedure. The number of data points, $N$ is chosen to minimize the model error based on the relationship

$$
\text { model error } \approx \sigma \frac{\sqrt{n}}{\sqrt{N}}
$$

where $\sigma$ represents the noise variance and $n$ represents the number of parameters to be estimated [51].

Encoder readings representing the angular displacements of both links from the stable equilibrium are stored in the MATLAB workspace. The differential equations that describe the plant dynamics given in Eqns. (3.4.5)-(5.3.5) are implemented using Simulink function blocks. The Simulink mathematical model is excited with the same chirp signal used on the 
real plant and a Runge-Kutta numerical integration algorithm solves for the resulting angular displacements $\hat{q}_{1}$ and $\hat{q}_{2}$. A constrained nonlinear least squares optimization algorithm, lsqnonlin, available in the MATLAB Optimization toolbox [46], minimizes the squared error, $e^{2}$, between the real and simulated output states where $e=\left(\hat{q}_{1}-q_{1}\right)^{2}+\left(\hat{q}_{2}-q_{2}\right)^{2}$, resulting to an optimal solution in $\theta$. The parameters are validated on fresh data and a measure of the model quality using the estimated parameters is obtained using the expression

$$
f i t=100\left(1-\frac{\sqrt{\sum_{i=1}^{N}\left(q_{x}(t)-\hat{q_{x}}(t)\right)^{2}}}{\sqrt{\sum_{i=1}^{N}\left(q_{x}(t)-\left(\bar{q}_{x}\right)\right)^{2}}}\right),
$$

where $x$ is 1 or 2 , depending on the angular position vector, and $N$ is the length of the data set.

The measurable system parameters are given in Table 3.1, while Figures 3.5 and 3.6 below, illustrate the effectiveness of the nonlinear system identification routine with $\phi$ set to $20^{\circ}$. The experiment is carried out with the system parameters given in Table 3.1. Fig. 3.5 represents the real and simulated data collected before the optimization routine. It can be observed that there is no correlation between the real and simulated data for link 1 , which is also confirmed by a negative fit of -1743 .

\subsection{Experimental Identification of $\phi_{o p t}$}

The objective of this thesis is to develop a balancing mechanism and control system that can maintain an upright posture, using minimal mechanical power, in a $2 \mathrm{DOF}$, one legged robot. The balancing mechanism comprises two links separated by an angle $\phi$ with concentrated masses at each end. In Section 2.7 of this thesis, the effect of a change in $\phi$ on the system power requirements was analyzed. It was observed that to achieve minimal mechanical power simply by altering the physical structure, $\phi$ will have to be set such that the linearized 
Table 3.1: Physical Parameters

\begin{tabular}{|l|l|l|}
\hline Symbol & Units & Value \\
\hline$m_{1}$ & $\mathrm{Kg}$ & 1.6330 \\
$m_{2}$ & $\mathrm{Kg}$ & 1.2300 \\
$l_{1}$ & $\mathrm{~m}$ & 0.4 \\
$l_{2}$ & $\mathrm{~m}$ & 0.37 \\
$\mathrm{~g}$ & $K g / m / s^{2}$ & 9.81 \\
$\mathrm{E}$ & $\mathrm{V}$ & 24 \\
$k_{a}$ & $\mathrm{~V} / \mathrm{V}$ & 2.4 \\
$T_{c}$ & $\mathrm{Nm}$ & 3.5 \\
$T_{P K}$ & $\mathrm{Nm}$ & 33 \\
$m_{m}$ & $\mathrm{Kg}$ & 1.017 \\
$R_{a}$ & $\Omega$ & 2.64 \\
$L_{a}$ & $\mathrm{mH}$ & 2.63 \\
$K_{m}$ & $\mathrm{~N}-\mathrm{m} / \mathrm{A}$ & 0.046 \\
$K_{e}$ & $\mathrm{~V} / \mathrm{rad} / \mathrm{s}$ & 0.046 \\
$T_{f}$ & $\mathrm{Nm}$ & $8.5 \mathrm{e}-3$ \\
$J_{m}$ & $K g-m^{2}$ & $1.6 \mathrm{e}-5$ \\
$g_{r}$ & - & $65.5: 1$ \\
$T_{s}$ & $\mathrm{~s}$ & 0.05 \\
\hline
\end{tabular}

system has transfer function zeros of relatively large magnitude, or no transfer function zeros at all. The value of $\phi$ that corresponds to the lack of transfer function zeros is referred to as $\phi_{o p t}$.

A mathematical expression for $\phi_{\text {opt }}$ was derived in (2.8.2) and given below:

$$
\phi=\phi_{o p t}=\cos ^{-1}\left(\frac{m_{2} l_{2}^{2}+I_{m} g_{r}}{m_{2} l_{1} l_{2}}\right)
$$

The derivation of Equations (3.5.1) is based on a simplified moment of inertia of the body about its center of gravity i.e.

$$
I_{L 2}=m_{2}\left(l_{2}^{2}-l_{c 2}^{2}\right)
$$

The above expression is further based on the simplifying assumption that the center of gravity of the body lies in line and midway between both link masses. These assumptions inadvertently lead to an inaccurate estimation of $\phi_{\text {opt }}$, which could prevent the realization of mechanical power savings. Moreover, $I_{L 2}$ is one parameter that cannot be accurately 
Table 3.2: Estimated Parameters

\begin{tabular}{|l|c|c|c|c|c|}
\hline$\phi\left(^{\circ}\right)$ & 20 & 30 & 40 & 50 & 60 \\
\hline$L_{c 1}(\mathrm{~m})$ & 0.0536 & 0.0503 & 0.0554 & 0.0631 & 0.0747 \\
$L_{c 2}(\mathrm{~m})$ & 0.0633 & 0.0526 & 0.0430 & 0.0400 & 0.0253 \\
$I_{L 1}\left(\mathrm{Kgm}^{2}\right)$ & 0.0218 & 0.0108 & 0.0095 & 0.0035 & 0.0199 \\
$I_{L 2}\left(\mathrm{Kgm}^{2}\right)$ & 0.0259 & 0.0212 & 0.0221 & 0.0252 & 0.0241 \\
$f_{r 1}(\mathrm{Nms} / \mathrm{rad})$ & 0.1953 & 0.1127 & 0.1438 & 0.1004 & 0.1000 \\
$f_{r 2}(\mathrm{Nms} / \mathrm{rad})$ & 0.4988 & 0.5000 & 0.5000 & 0.4999 & 0.5000 \\
$f_{c 1}(\mathrm{Nm})$ & 0 & 0.0186 & 0.0081 & 0.0219 & 0.0040 \\
$f_{c 2}(\mathrm{Nm})$ & 0.7271 & 0.9269 & 0.7555 & 0.6343 & 0.2156 \\
$\eta$ & 0.2743 & 0.3092 & 0.2668 & 0.2537 & 0.1744 \\
\hline
\end{tabular}

measured or computed and will have to be estimated via system identification. To obtain a more accurate value for $\phi_{\text {opt }}$, system identification is, therefore, performed for several values of $\phi$. The resulting parameters are then employed with the linearized model, and the locus of the transfer function zeros is used to obtain a better estimate of $\phi_{\text {opt }}$.

Figure 3.6, on the other hand, represents the real and simulated data collected after the optimization routine. It can be seen that the optimization routine successfully converges to a good solution as the simulated data correlates to the real data. The fit has also dramatically improved for link 1 and for link 2 to $88 \%$ and $92 \%$ respectively.

To obtain a more accurate value for $\phi_{\text {opt }}$, system identification is performed for several values of $\phi$. The resulting parameters are then employed with the linearized model and the locus of the transfer function zeros is used to obtain a better estimate of $\phi_{\text {opt }}$.

Specifically, system identification was performed for 6 settings of $\phi: \phi=20^{\circ}, 30^{\circ}, 40^{\circ}$, $50^{\circ}, 60^{\circ}$. Table 3.2 below shows the parameters estimated from the nonlinear gray box identification routine, while Tables 3.3 and 3.4, respectively, display the fit before and after system identification. Figures 3.7 to 3.14 illustrate the results of the system identification for each individual selection of $\phi$. The plotted results as well as the resulting goodness measures further illustrate the effectiveness of the identification procedure.

Using the estimated parameters given in Table 3.2, the transfer function zeros of the 

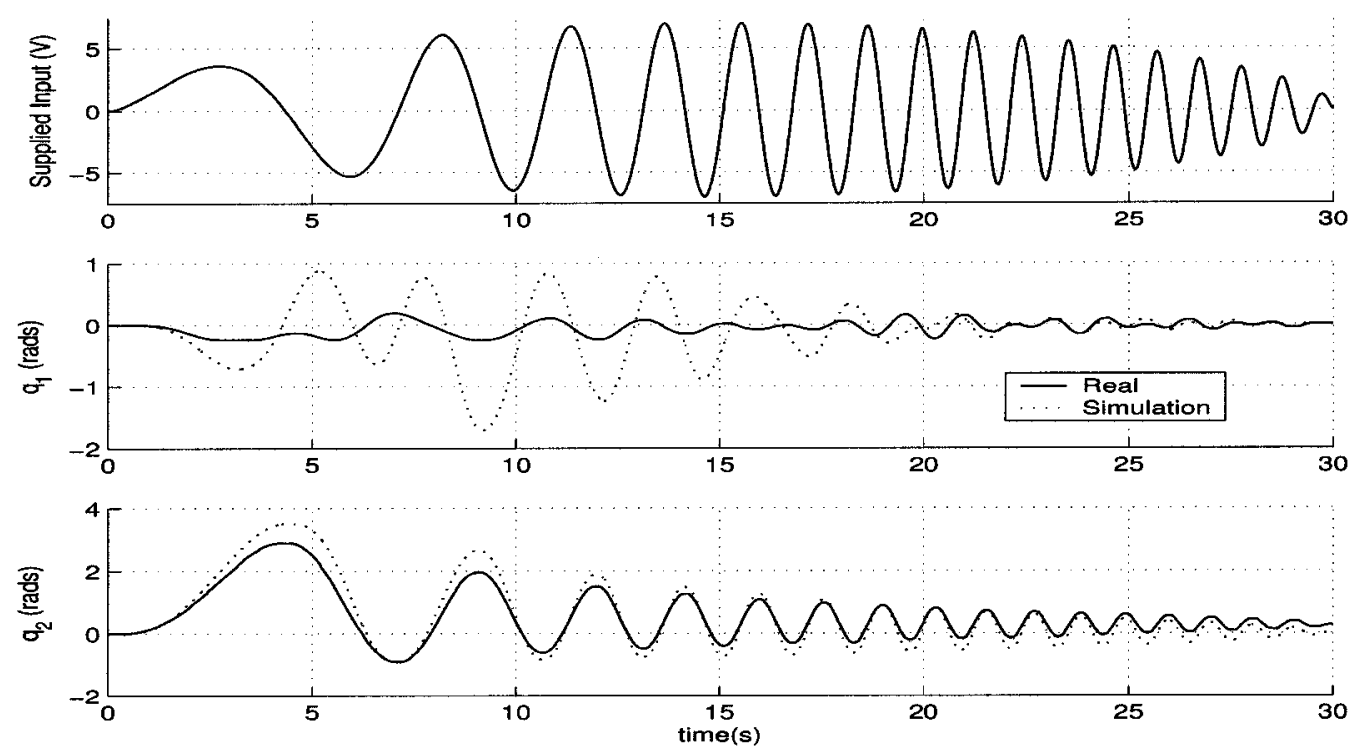

Figure 3.5: Comparison between real and simulation data for $\phi=20^{\circ}$ using raw data, Fit $=$ $-1743 ; 85.03$
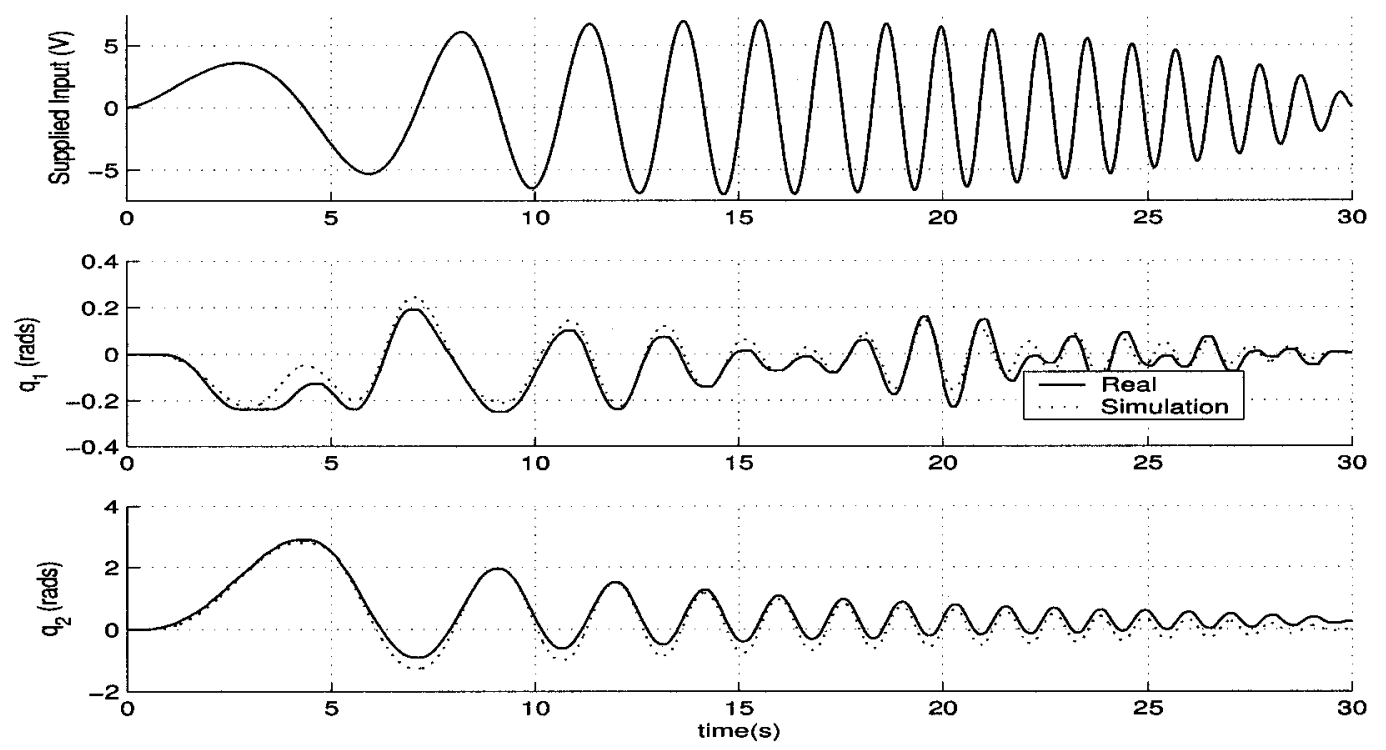

Figure 3.6: Comparison between real and simulation data for $\phi=20^{\circ}$ using estimated data, Fit $=$ $88.0846 ; 92.2398$ 

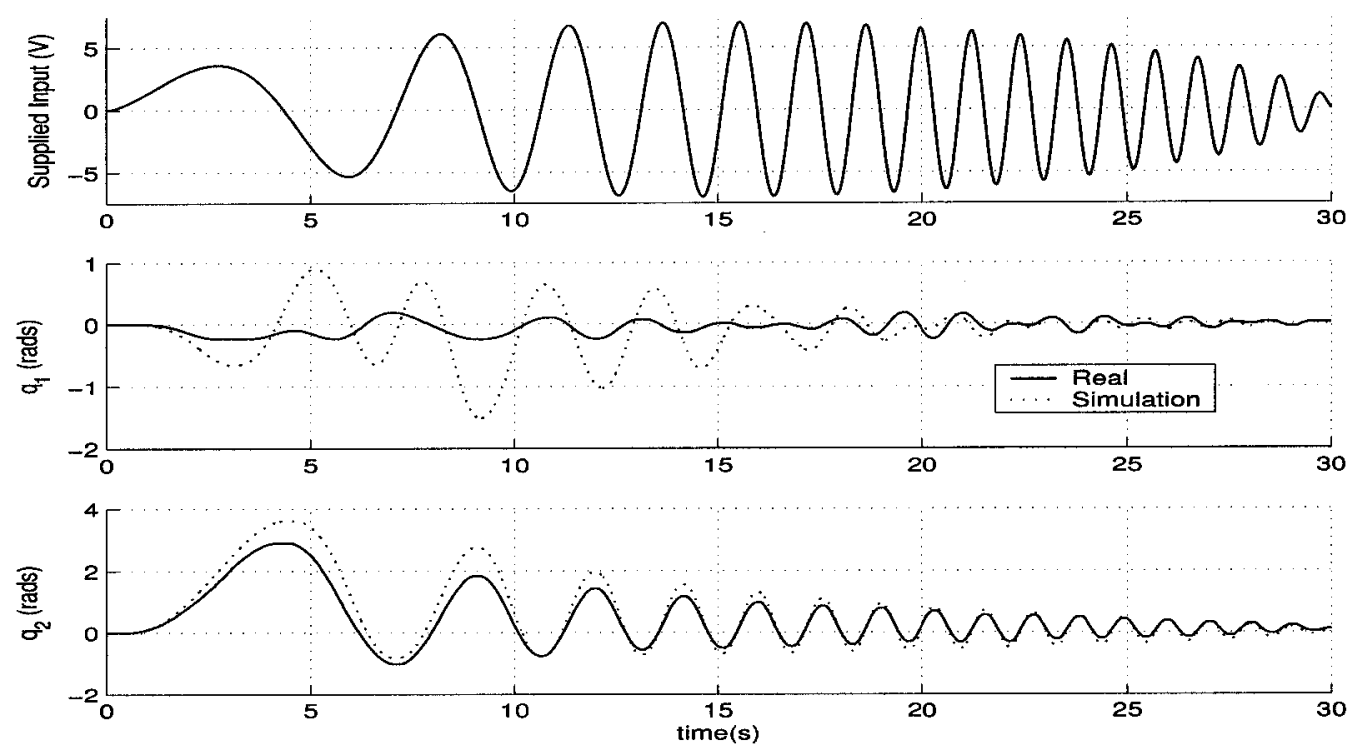

Figure 3.7: Comparison between real and simulation data for $\phi=30^{\circ}$ using raw data, Fit $=$ $-1386 ; 82.9$
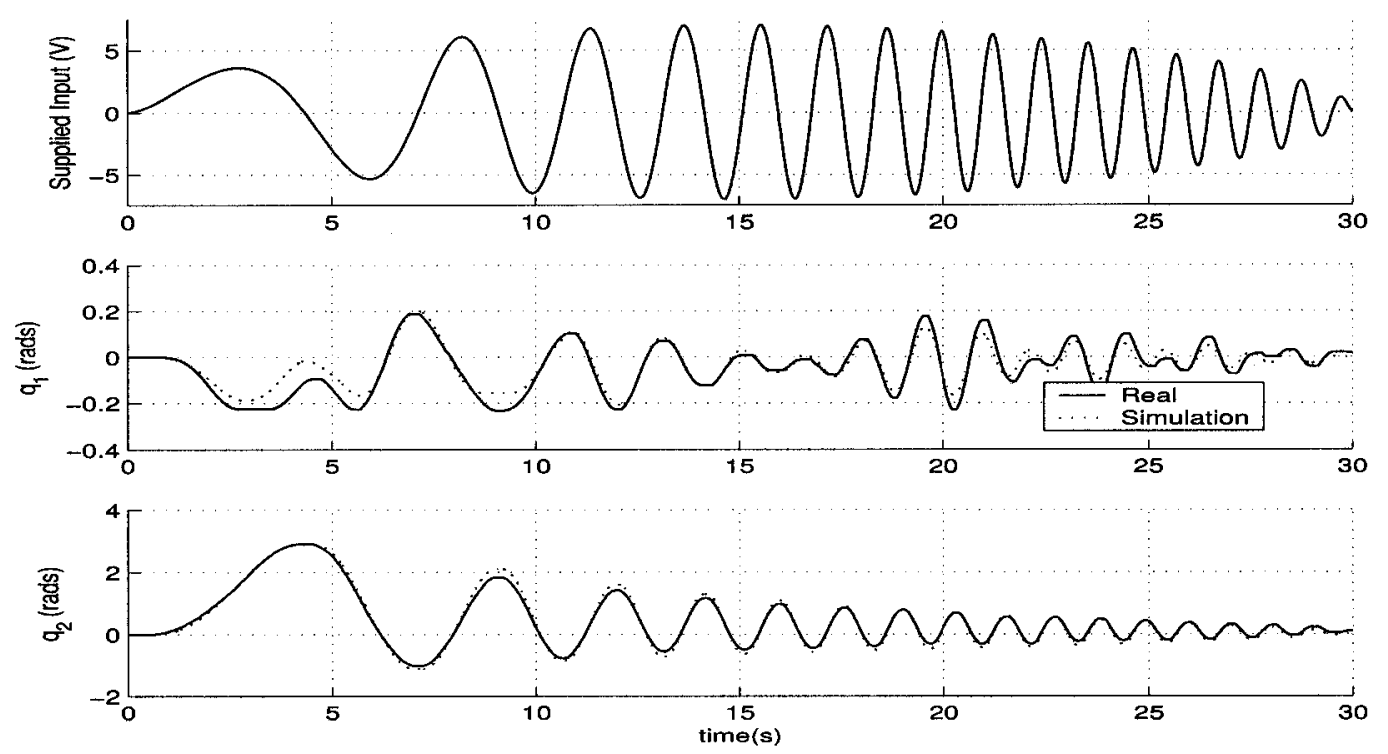

Figure 3.8: Comparison between real and simulation data for $\phi=30^{\circ}$ using estimated data, Fit $=$ $87.8 ; 98.6$ 

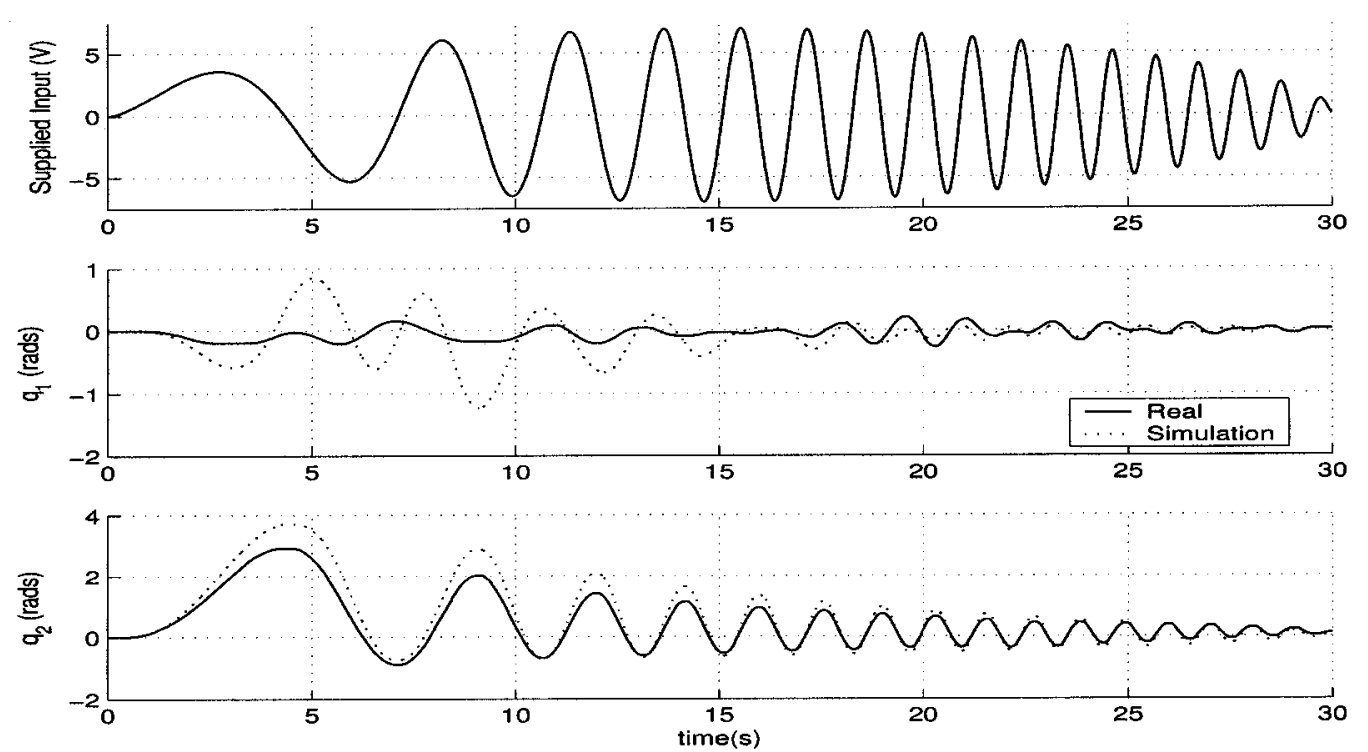

Figure 3.9: Comparison between real and simulation data for $\phi=40^{\circ}$ using raw data, Fit $=$ $-97.9 ; 82.0$
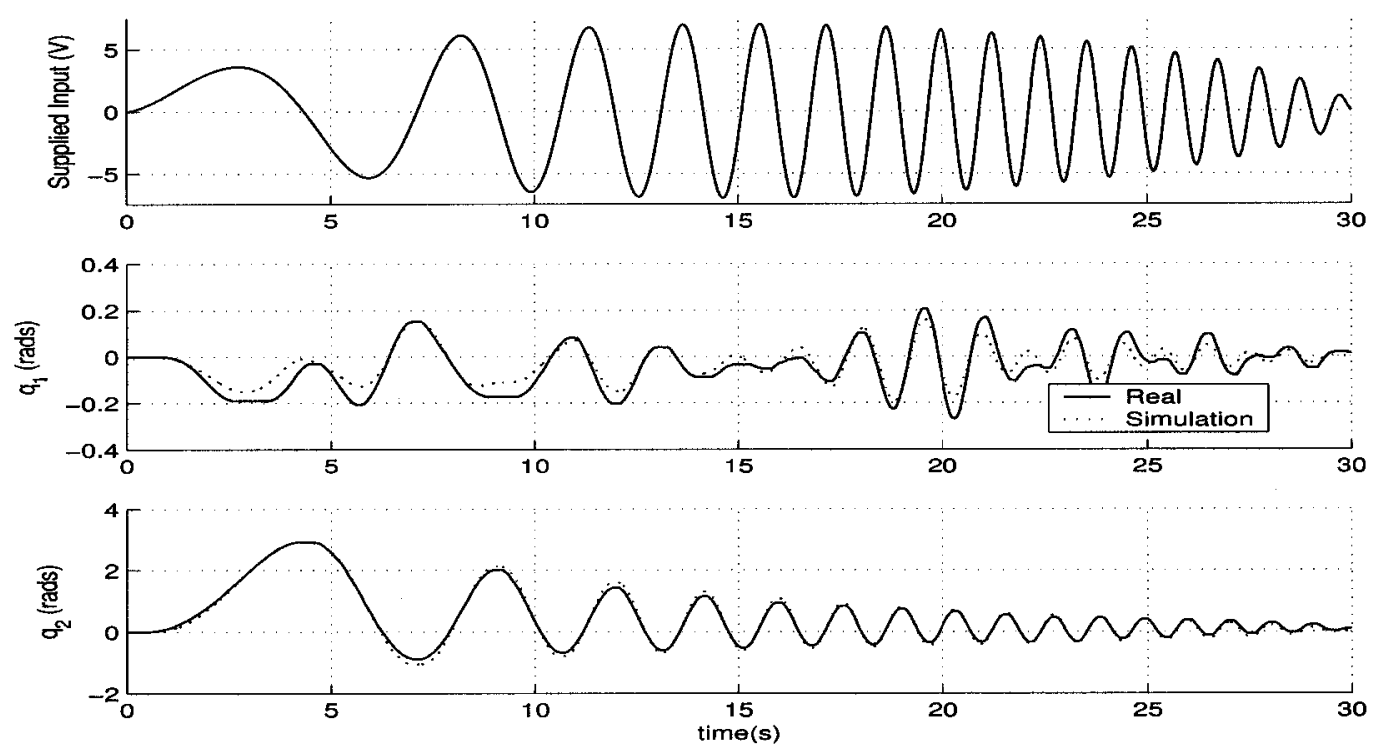

Figure 3.10: Comparison between real and simulation data for $\phi=40^{\circ}$ using estimated data, Fit $=83.3 ; 99.1$ 

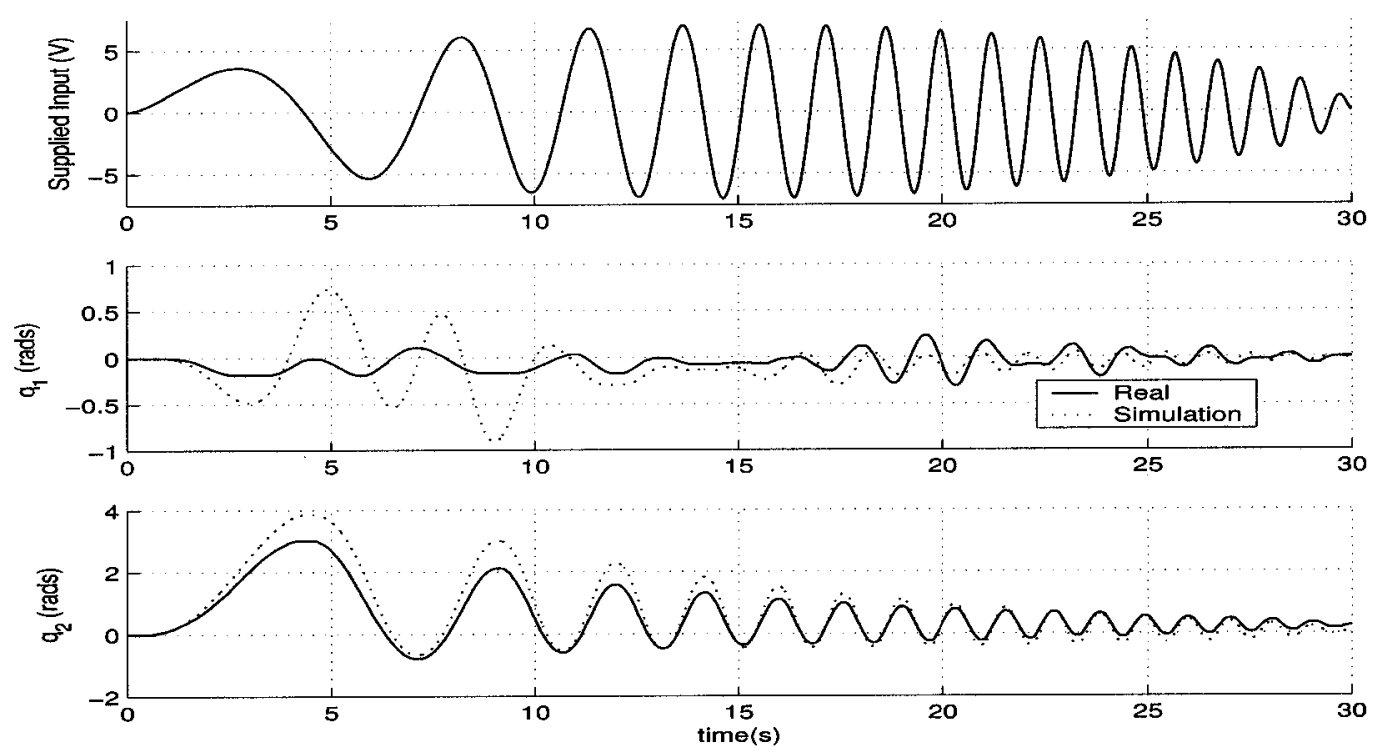

Figure 3.11: Comparison between real and simulation data for $\phi=50^{\circ}$ using raw data, Fit $=$ $-561.6 ; 80.5$
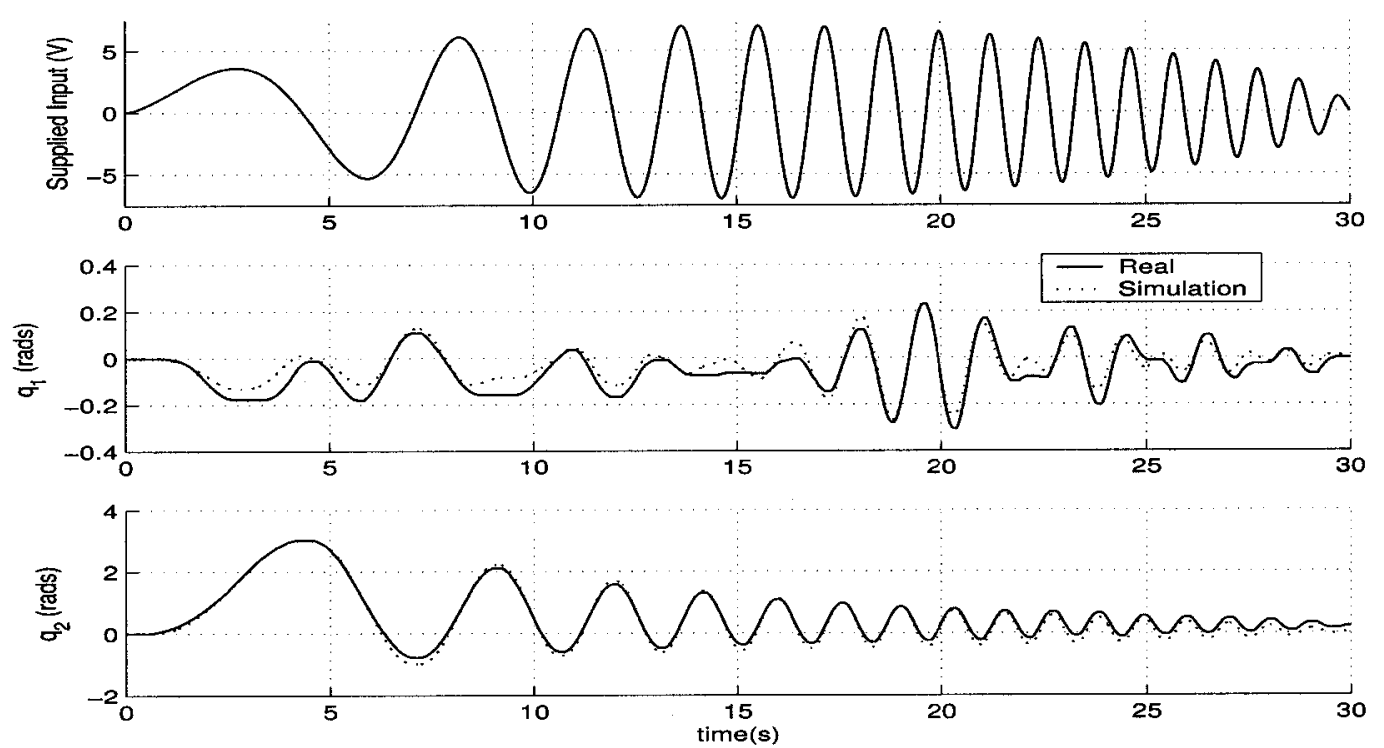

Figure 3.12: Comparison between real and simulation data for $\phi=50^{\circ}$ using estimated data, Fit $=82.2 ; 97.8$ 

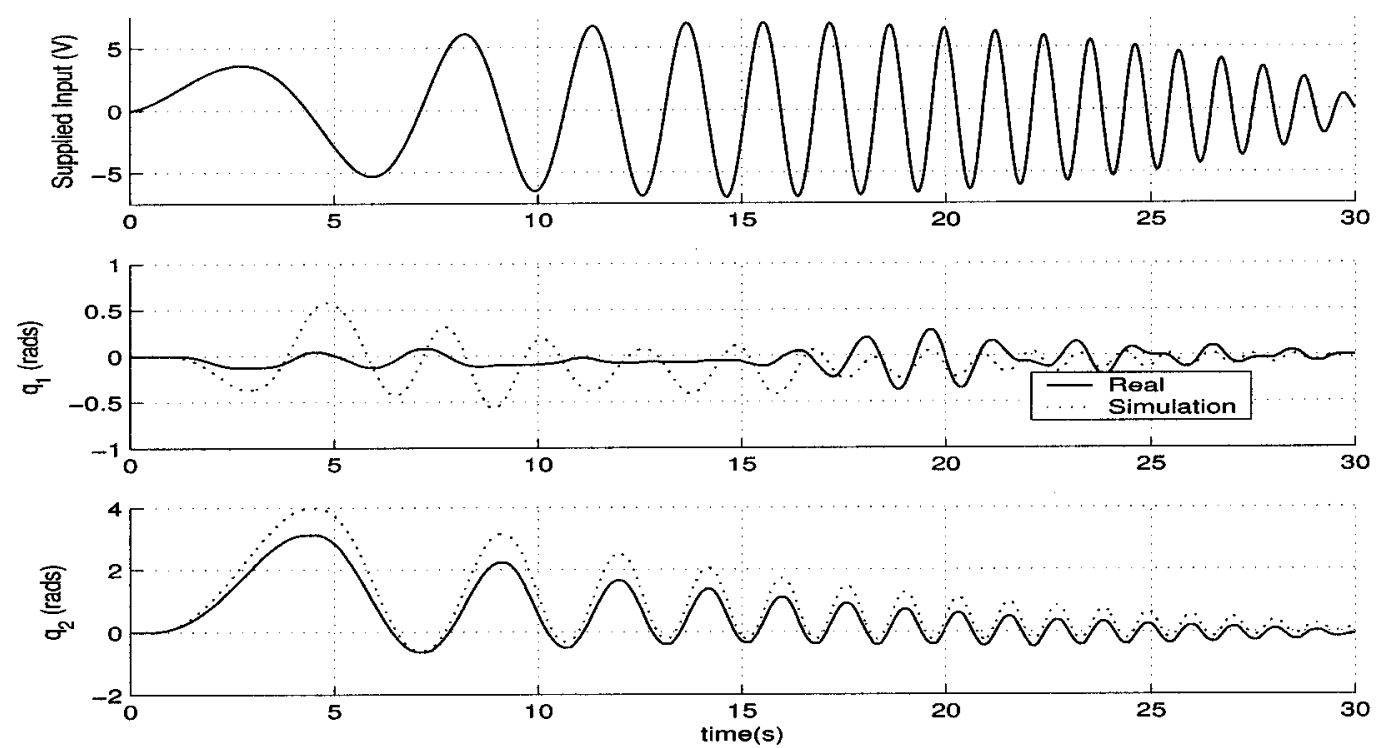

Figure 3.13: Comparison between real and simulation data for $\phi=60^{\circ}$ using raw data, Fit $=$ $-344.4 ; 76.7$
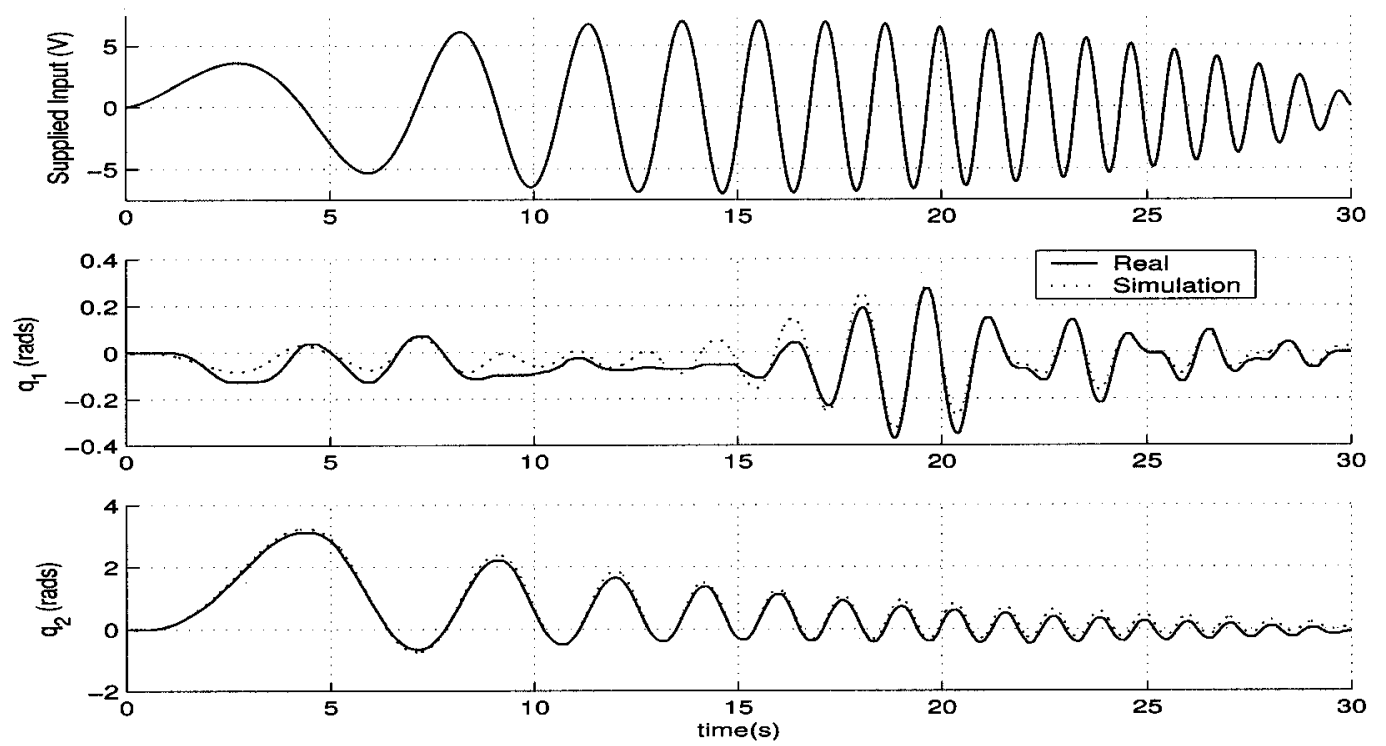

Figure 3.14: Comparison between real and simulation data for $\phi=60^{\circ}$ using estimated data, Fit $=83.3 ; 98.0$ 
Table 3.3: Fit Values Before System Identification

\begin{tabular}{|l|c|c|c|c|c|}
\hline$\phi\left(^{\circ}\right)$ & 20 & 30 & 40 & 50 & 60 \\
\hline Link 1 & -1743.6 & -1386.8 & -97.9 & -561.6 & -344.4 \\
Link 2 & 85.0 & 82.96 & 82.0 & 80.5 & 76.7 \\
\hline
\end{tabular}

Table 3.4: Fit Values After System Identification

\begin{tabular}{|l|c|c|c|c|c|}
\hline$\phi\left(^{\circ}\right)$ & 20 & 30 & 40 & 50 & 60 \\
\hline Link 1 & 88.1 & 87.8 & 83.3 & 82.2 & 83.3 \\
Link 2 & 92.2 & 98.6 & 99.1 & 97.8 & 98.0 \\
\hline
\end{tabular}

linearized system are plotted for the various values of $\phi$. As indicated in Figure 3.15, the system is non-minimum phase for $\phi=20^{\circ}$ and $30^{\circ}$, as there is a transfer function zero on the positive real axis on the s-plane, at each of these settings. For $\phi=40^{\circ}, 50^{\circ}$ and $60^{\circ}$ the system is marginally minimum phase for transfer function zeros lie along the imaginary axis. Therefore, there exists a value of $\phi=\phi_{\text {opt }}$ such that the transfer function zeros are of very large magnitude or nonexistent, that is, between $30^{\circ}$ and $40^{\circ}$. In other words, $30^{\circ} \leq \phi_{\text {opt }} \leq 40^{\circ}$. For the sake of comparison, the mathematical value of $\phi_{\text {opt }}$ is computed using Equation (2.8.2) is $21.5^{\circ}$. Several sources can be cited for this discrepancy. The predominant contributor, however, lies within the simplification of the system dynamics and the assumptions that had to be made prior to formulating the nonlinear system dynamics. Nonetheless, this results further substantiates the theory behind an optimum $\phi$ that will render the linearized system minimum phase (i.e. no transfer function zeros). The physical design only allows discrete selection of angles in multiples of $10^{\circ}$. As such, a $\phi_{o p t}$ is set to $40^{\circ}$ for the rest of the project.

\subsection{Sensitivity Analysis}

With $\phi$ set to $40^{\circ}$, a sensitivity analysis is performed for each individual parameter. The procedure involves finding the parameter that produces the greatest deviation from the real model when multiplied by an uncertainty factor. A $+40 \%$ uncertainty is applied to all the 


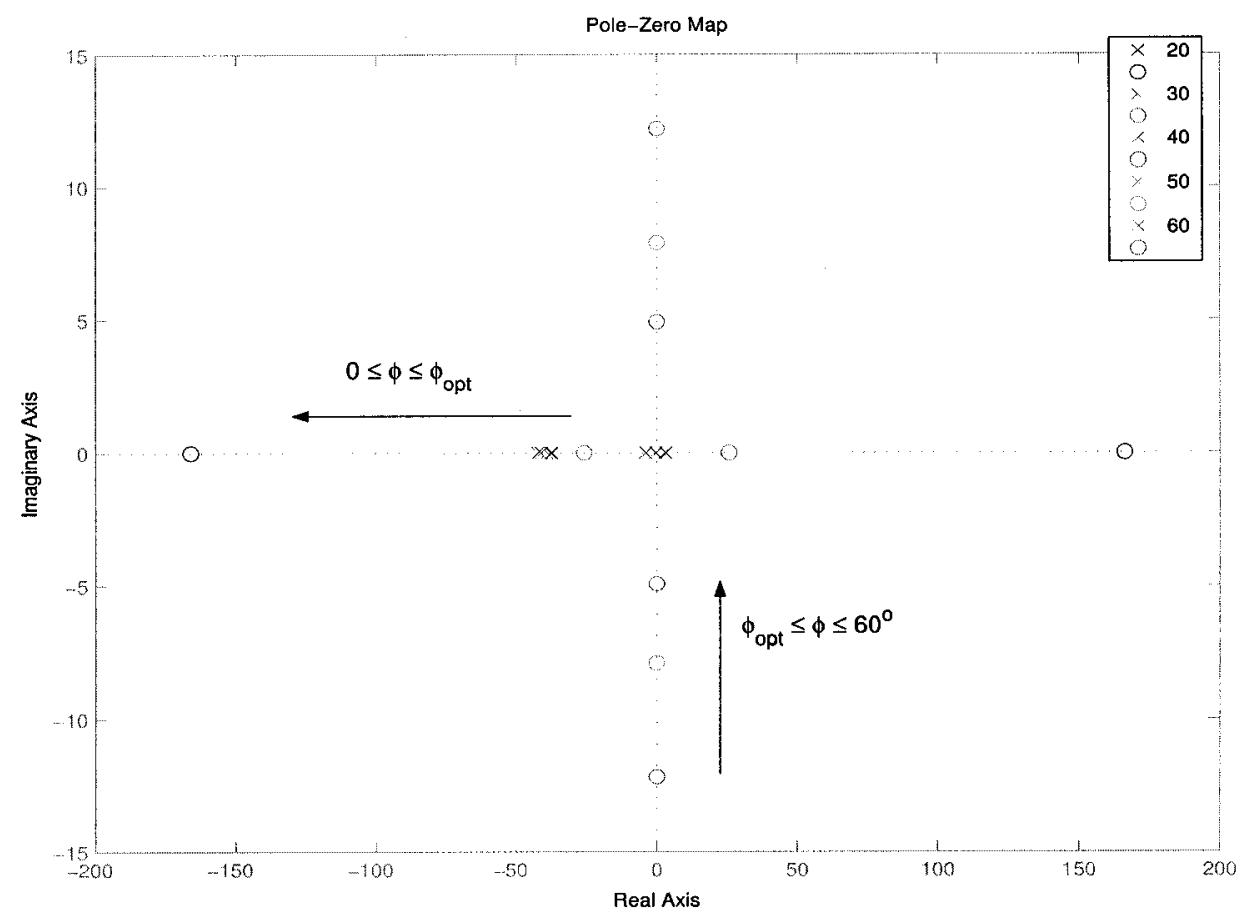

Figure 3.15: Pole-Zero locus using estimated parameters

Table 3.5: Model fit changes for a $40 \%$ parametric uncertainty

\begin{tabular}{|l|c|c|c|c|c|c|c|c|c|}
\hline Parameter & $l_{c 1}$ & $l_{c 2}$ & $I_{L 1}$ & $I_{L 2}$ & $f_{r 1}$ & $f_{r 2}$ & $f_{10}$ & $f_{20}$ & $\eta$ \\
\hline Link 1 & -4.87 & -38.35 & -0.13 & -26.06 & -1.51 & -2.42 & -0.08 & -7.12 & -53.19 \\
Link 2 & -0.01 & -2.33 & -0.00 & -0.01 & -0.01 & -0.89 & -0.00 & -3.02 & -17.07 \\
\hline
\end{tabular}

parameters estimated in the previous section and the changes in the data fit are recorded as illustrated in Table 3.5. Each column in the table represents the change in the model fit when the corresponding parameter is multiplied by a $+40 \%$ uncertainty. For instance, column 10 represents the change in the model fit when the parameter $\eta$ is multiplied by a $40 \%$ uncertainty. It can be observed that this uncertainty in this parameter introduces the largest modelling uncertainty. The same pattern is observed for negative parametric uncertainty. 


\subsection{Conclusion}

In this chapter, a customized nonlinear gray-box system identification procedure was successfully used to estimate certain parameters in the dynamic model of the one-legged robotic prototype. The estimated parameters are either impossible or difficult to measure and compute mathematically, forcing their estimation using input-output data. An optimal design angle, referred to as $\phi_{\text {opt }}$, which was defined in Section 2.7, qualifies as a parameter whose mathematical computation is flawed from over simplification of nonlinear phenomena inherent in the real system. Consequently, this important, yet un-measurable, system parameter was estimated using a nonlinear identification procedure, and was found to lie within the neighborhood of $30^{\circ}$ and $40^{\circ}$. Given that the physical design only allows discrete selection of angles in multiples of $10^{\circ}, \phi$ is set to $40^{\circ}$ for the remainder of the project.

Furthermore, a sensitivity analysis was also performed on the estimated model parameters. It was observed that uncertainty in $l_{c 2}$ and $\eta$ largely account for modelling discrepancy. 


\section{Chapter 4}

\section{Linear Quadratic Regulator (LQR) with Integral Action}

\subsection{Introduction}

This chapter presents the first attempt to design a model-based controller to maintain an upright posture on a one legged monopod. The chapter begins with definition of the control objective followed by a state space description of the plant dynamics linearized about the unstable equilibrium. Following thereafter is the synthesis of a Linear Quadratic Regulator with Integral action. The chapter is concluded with the presentation of simulation and experimental results based on the closed loop performance of the system.

\subsection{Control Objectives}

The goal of this thesis is to develop a balancing mechanism and control system that can maintain an upright posture, using minimal mechanical power, in a 2 DOF, one legged robot. The system exhibits numerous nonlinear phenomena, is inherently open-loop unstable and non-minimum phase, making the control task a challenging one. The non-minimum phase 
behavior, which is a direct consequence of the existence of a transfer function zero in the right half plane of the s-plane, contributes to an undesirable inverse response [52, 231]. In section 2.7 of this thesis, the effect of a change in body curvature, the latter represented by the parameter $\phi$, on system power requirements was analyzed. It was observed that the conservation of mechanical power or control effort could be achieved simply by altering $\phi$. The net effect turns out to be a gradual transition of the transfer function zeros from the real to the imaginary $(\mathrm{j} \omega)$ axis of the s-plane. The theoretical value of $\phi$ where this transition occurs is referred to as $\phi_{\text {opt }}$. At $\phi_{\text {opt }}$, the zeros of are relatively large magnitude or can be considered absent; hence, the control system will not have to deal with the detrimental contribution characterized by an inverse response. However, given that $\phi_{\text {opt }}$ is not physically realizable, as demonstrated in Section $3.5, \phi$ is set to $40^{\circ}$. At this setting, the system is considered marginally minimum phase, as the adverse contributions of imaginary axis transfer function zeros are not as severe. See Section 2.7 for an explanation.

The objective of the control system is to force the system outputs $q_{1}$ and $q_{2}$ to zero, or within a close neighborhood thereof, while being insensitive to actuator saturation and uncertainties in the plant dynamics. In relation to existing physical realizations of controlling hopping robots, this task is to be achieved using less control effort. The outputs of interest $q_{1}$ and $q_{2}$ represent the angular displacement of the leg from the vertical axis and an angular displacement of the body from the leg axis, respectively.

Two controller types are synthesized to achieve the control objectives. The Linear Quadratic Regulator (LQR) optimal control and a nonlinear controller based on Feedback Linearization. To reduce steady state error, integral action is forced into the solution of the controllers. This chapter deals exclusively with the synthesis and experimental validation of the LQR control law in terms of achieving the control objectives of this project. 


\subsection{State Space Description of Acrobot}

The first step in designing a model-based controller is to obtain a linear model of the system about the equilibrium position of interest. The linearized model allows the control engineer to gain a better understanding of the system behavior around the equilibrium point and also serves as a platform for controller synthesis, as will be seen later in this chapter. As described in Section 2.5, the system under investigation in this thesis has a continuous equilibrium manifold defined by the expression (2.5.4). Using this expression and the estimated parameters obtained in Chapter 3 allows one to plot the equilibrium manifold as given in Figure 4.1. Furthermore, the control input required to maintain each equilibrium state in Figure 4.2.

The equilibrium point of interest ${ }^{1}$ lies on the origin of the plots shown in Figures 4.1 and 4.2. This corresponds to $q_{10}=q_{20}=0$ and $u_{e q}=0$ (ignoring the slight offset in the motor input voltage), where $q_{k 0}$ represents an equilibrium state of each link $k$. Recall that at each equilibrium state, the angular velocity for each link is zero (i.e. $\dot{q}_{k 0}=0$ ). The linear model of the system is obtained by linearizing the original dynamics about this unstable operating point. Defining an $n \times 1$ state matrix $\mathbf{x}=\left[\begin{array}{llll}q_{1} & q_{2} & \dot{q}_{1} & \dot{q}_{2}\end{array}\right]$ and recalling the results from Section 2.6 gives the linearized continuous-time model as follows:

$$
\begin{aligned}
& \dot{\mathbf{x}}=\mathbf{A}_{\mathbf{o}} \mathbf{x}+\mathbf{B}_{\mathrm{o}} u \\
& \mathbf{y}=\mathbf{C}_{\mathrm{o}} \mathbf{x}+D_{o} u
\end{aligned}
$$

\footnotetext{
${ }^{1}$ also referred to as the unstable operating point
} 


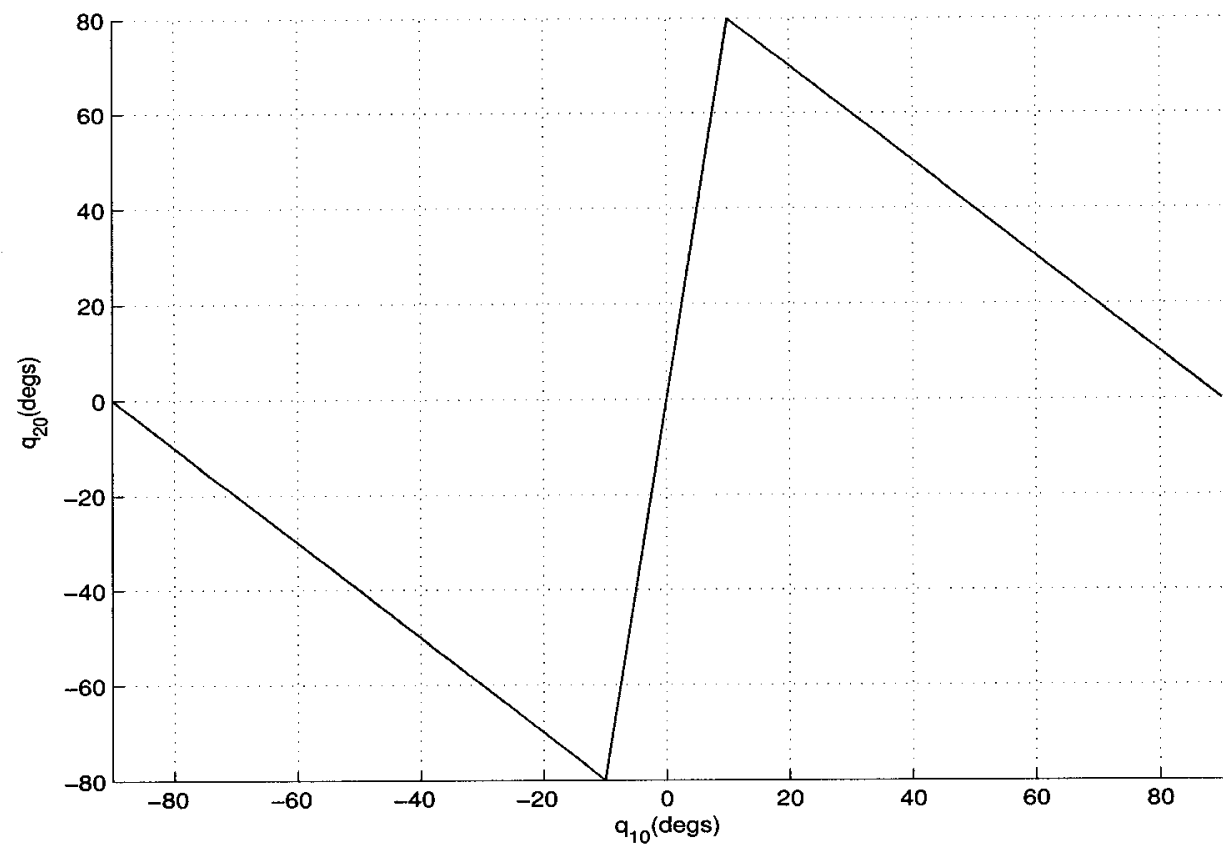

Figure 4.1: The Equilibrium Manifold

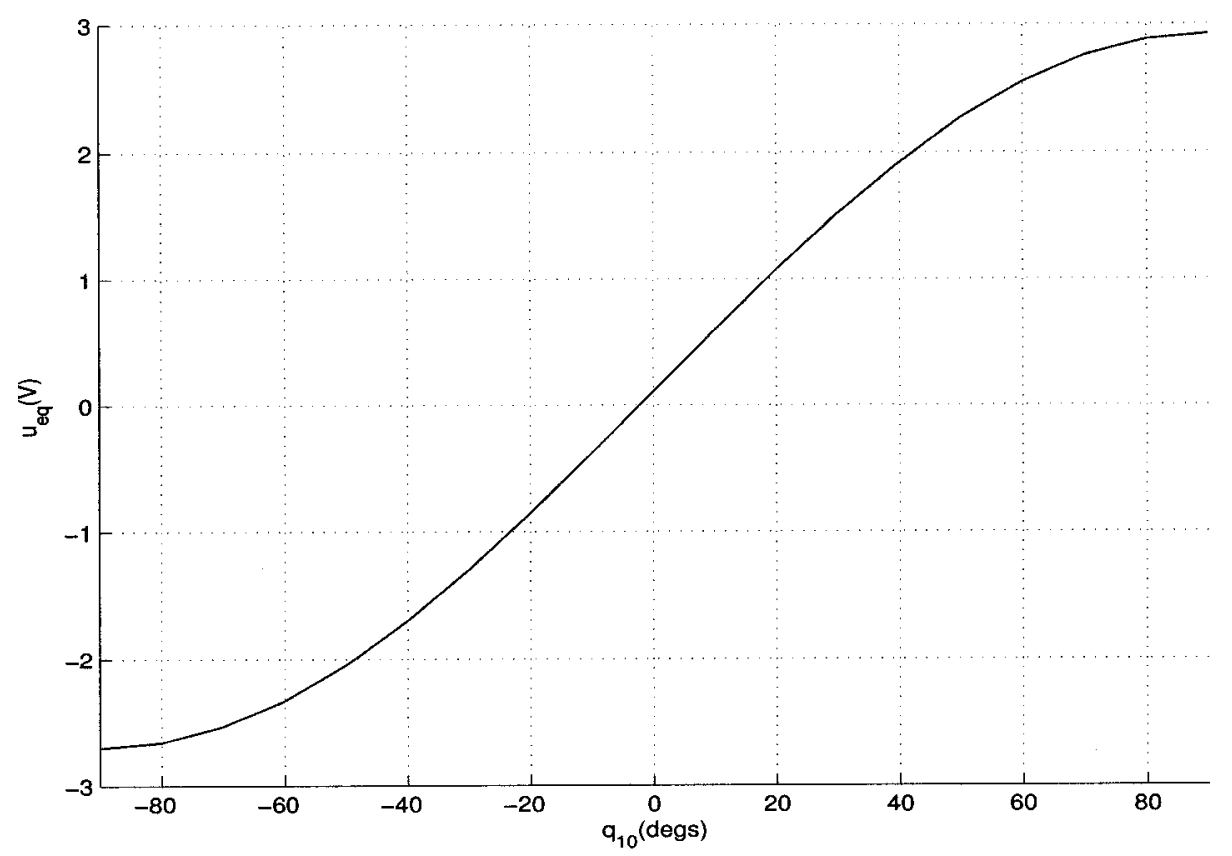

Figure 4.2: Equilibrium Input Voltage to Actuator 
where

$\mathbf{A}_{o}=\left[\begin{array}{cccc}0 & 0 & 1 & 0 \\ 0 & 0 & 0 & 1 \\ \frac{m_{16}\left(m_{3}-m_{1}\right)+m_{13}\left(m_{5}-m_{6}\right)}{\Delta} & \frac{m_{16}\left(m_{3}-m_{1}\right)-m_{13} m_{6}}{\Delta} & \frac{-m_{1} 3 f_{r 1}}{\Delta} & \frac{m_{14}\left(m_{3}-m_{1}\right)}{\Delta} \\ \frac{\left(m_{5}+m_{6}\right)\left(m_{12}-m_{11}\right)+m_{16}\left(2 m_{1}-m_{2}\right)}{\Delta} & \frac{m_{6}\left(m_{11}-m_{12}\right)+m_{16}\left(2 m_{1}-m_{2}\right)}{\Delta} & \frac{f_{r 1}\left(m_{11}-m_{12}\right.}{\Delta} & \frac{-m_{14}\left(m_{2}-2 m_{1}\right)}{\Delta}\end{array}\right]$

$$
\mathbf{B}_{o}=\left[\begin{array}{c}
0 \\
0 \\
\frac{-m_{3}+m_{1}}{\Delta} \\
\frac{m_{2}-2 m_{1}}{\Delta}
\end{array}\right] \quad \mathbf{C}_{o}=\left[\begin{array}{llll}
1 & 0 & 0 & 0
\end{array}\right] \quad D_{o}=0
$$

$$
\Delta=\left(m_{12}--m_{11}\right)\left(m_{3}-m_{1}\right)+m_{13}\left(m_{2}-2 m_{1}\right)
$$

and the constants $m_{i}, i=1: 16$ can be found defined in Section 2.4 or Equation (3.4.10). The matrices $\mathbf{A}_{\mathbf{o}}, \mathbf{B}_{\mathbf{o}}$, and $\mathbf{C}_{\mathbf{o}}$ given in Equations (4.3.2) and (4.3.3) are typically referred to as the system, input distribution, and output distribution matrices, but for convenience, the set $\left(\mathbf{A}_{\mathbf{o}}, \mathbf{B}_{\mathbf{o}}, \mathbf{C}_{\mathbf{o}}\right)$ will henceforth be referred to as the system triple. Note that dependency of state and control on the time vector $t$ has been omitted for the sake of clarity.

\subsection{Controller Design}

Consider the single input stabilizable LTI system represented by (4.3.1) with initial conditions $\mathbf{x}\left(\mathbf{t}_{\mathbf{0}}\right)=\mathbf{x}_{0}$. We seek the optimal State-Feedback control of the form

$$
u(t)=-\mathbf{K}_{L Q R} \mathbf{x}(t)
$$

where $\mathbf{K}_{L Q R}$ is computed such that the following cost function is minimized:

$$
V(\mathbf{x}, u)=\int_{0}^{\infty} \mathbf{x}^{T} \mathbf{Q} \mathbf{x}+u^{T} \mathbf{R} u d t
$$

with $\mathbf{Q}=\mathbf{Q}^{T} \geq 0 \in \Re^{n \times n}$, where $n$ represents the dimension of the state vector, and $R>0 \in \Re^{m}$, where $m$ represents the dimension of the control vector. For scalar control, 
$m=1$. Minimization of $V$ implies forcing the state vector $\mathbf{x}$ to 0 with as little control energy and state deviation as possible with the balance between control effort and state deviation dictated by the $\mathbf{Q}$ and $\mathbf{R}$ matrices.

The optimal solution to (4.4.2) is given by (4.4.5) with

$$
\mathbf{K}_{L Q R}=R^{-1} \mathbf{B}_{\mathbf{o}} \mathbf{P}
$$

where $\mathbf{P}$ satisfies the Continuous time Algebraic Ricatti Equation (CARE)

$$
0=\mathbf{Q}-\mathbf{P} \mathbf{B}_{\mathbf{o}} R^{-1} \mathbf{B}_{\mathbf{o}}{ }^{T} \mathbf{P}+\mathbf{P} \mathbf{A}_{\mathbf{o}}+\mathbf{A}_{\mathbf{o}}{ }^{T} \mathbf{P}
$$

If the set $\left(\mathbf{A}_{\mathbf{o}}, \mathbf{B}_{\mathbf{o}}\right)$ is stabilizable and $\left(\mathbf{A}_{\mathbf{o}}, \mathbf{Q}^{1 / 2}\right)^{2}$ is detectable, then $P \geq 0$ and the closed loop system, given by

$$
\dot{x}=\left(\mathbf{A}_{\mathbf{o}}-\mathbf{B}_{\mathbf{o}} \mathbf{K}_{L Q R}\right) \mathbf{x}
$$

is stable ${ }^{3}$ and the closed loop poles can be allocated to any desirable position by the appropriate choice of $\mathbf{K}_{L Q R}$.

The square matrix $\mathbf{Q}$ and scalar $R$ (for a single input system), respectively represent the state and control cost, allowing the control engineer to also optimize control effort while attempting to meet the design specifications. A large value of $R$ relative to the largest diagonal elements of $Q$, for instance, imposes a high control penalty. Such a setting will prevent saturation of the control input at the expense of high excursions of state. On the other hand, making the diagonal elements of $Q$ much larger than $R$ places a high penalty on large excursions of states at the expense of control.

LQ control does not guarantee integral action; therefore, constant disturbances that contribute to steady state error, such as static friction, may not be rejected in steady state. To reject the steady state error, Integral action needs to be forced into the solution of the

\footnotetext{
${ }^{2}$ The term $\mathbf{Q}^{\mathbf{1 / 2}}$ represents any matrix that satisfies $\left(\mathbf{Q}^{\mathbf{1 / 2}}\right)^{T} \mathbf{Q}^{\mathbf{1 / 2}}=Q$

${ }^{3}$ Stability here means that the states remain bounded during regulation
} 
controller. This will be done by including a state integrator at the controller input and considering the regulator problem for the augmented system. Denoting the output variable $y=x_{1}$, the additional state $x_{c}$ is defined by the relationship

$$
\begin{aligned}
& \mathbf{x}_{\mathbf{a}}=\left[\begin{array}{l}
x_{c} \\
\mathbf{x}
\end{array}\right] \\
& \dot{x}_{c}=y .
\end{aligned}
$$

It can easily verified that the augmented system becomes:

$$
\begin{aligned}
& \dot{\mathbf{x}}_{\mathbf{a}}=\mathbf{A} \mathbf{x}_{\mathbf{a}}+\mathbf{B} u \\
& \mathbf{y}=\mathbf{C x}_{\mathbf{a}}+D u
\end{aligned}
$$

where

$$
x_{a}=\left[\begin{array}{c}
x_{\mathrm{c}} \\
\mathbf{x}
\end{array}\right] \quad A=\left[\begin{array}{ll}
0 & \mathbf{C}_{\mathrm{o}} \\
0 & \mathbf{A}_{\mathrm{o}}
\end{array}\right] \quad B=\left[\begin{array}{c}
0 \\
\mathbf{B}_{\mathrm{o}}
\end{array}\right] \quad C=\left[\begin{array}{ll}
0 & \mathbf{C}_{\mathrm{o}}
\end{array}\right] .
$$

The LQR problem is then applied as usual to the system triple $(\mathbf{A}, \mathbf{B}, \mathbf{C})$. The following observations can be made from Figures 4.1 and 4.2: a control system, during the process of driving the output $q_{1}$ to $q_{10}=0$ as defined by the control objectives, will inadvertently drive the state variable $q_{2}$ to $q_{20}=0$. As such, the system can be considered a SISO for the purposes of this exercise. This explains why the state $q_{2}$ is not accounted for in the output distribution matrix, $C$, as well as the input matrix, $D$, in Equation(4.4.8) above.

The physical design only allows for the selection of $\phi$ in multiples of $10^{\circ}$. To realize mechanical power savings as discussed in the previous chapter, $\phi_{\text {opt }}$ is set to $40^{\circ}$ for the remainder of the project. At this setting of $\phi$, the system is considered marginally minimum phase, as the adverse contribution of imaginary axis transfer function zeros are not as severe. In more practical terms, a relatively insignificant amount of control effort will be expended to correct the system's inverse response that would otherwise arise when transfer function zeros are on the real axis. See Section 2.7 for further details. 
Setting $\phi$ to $40^{\circ}$ and using the system parameters given in Table 3.1 leads to the following system triple:

$$
\begin{aligned}
& \mathbf{A}=\left[\begin{array}{ccccc}
0.00 & -1.00 & 0.00 & 0.00 & 0.00 \\
0.00 & 0.00 & 0.00 & 1.00 & 0.00 \\
0.00 & 0.00 & 0.00 & 0.00 & 1.00 \\
0.00 & 13.32 & -2.1055 & -0.62 & 0.62 \\
0.00 & -9.27 & -8.3846 & 0.036 & -41.38
\end{array}\right] \quad \mathbf{B}=\left[\begin{array}{c}
0.00 \\
0.00 \\
0.00 \\
-0.33 \\
21.92
\end{array}\right] \\
& \mathbf{C}=\left[\begin{array}{lllll}
0 & 1 & 0 & 0 & 0
\end{array}\right]
\end{aligned}
$$

The poles of the open loop continuous-time system are located at $-32.91,-0.30,-3.37$, 0.00 , and 3.74 indicating that the open loop system is inherently unstable as expected. Note that the pole at the origin results from the augmented system state. System zeros are located at \pm 12.18 i.

\subsection{Discretization}

The control system is a digital implementation. That is, the incremental optical encoder output is digitized using a DAQ PCI 6036E A/D converter through sampling and quantization. The digital data is then passed to the control algorithm that is executed by MATLAB Real Time Windows Target(RTW). The result from the control algorithm is converted to an analog signal by a NI DAQ PCI $6036 \mathrm{E}$ D/A sample and hold circuit and sent to the actuator, as illustrated in Figure 4.3. Note that there is a finite interval, albeit small, between when the angular positions are obtained and when corrective action is performed by the controller. To simplify our analysis, this interval is assumed to be negligible and it is further assumed that the sampling rate $T$ defines the fixed interval within which sensor data and control action is concurrently performed.

Besides converting system signals to discrete form, the discretization process introduces 


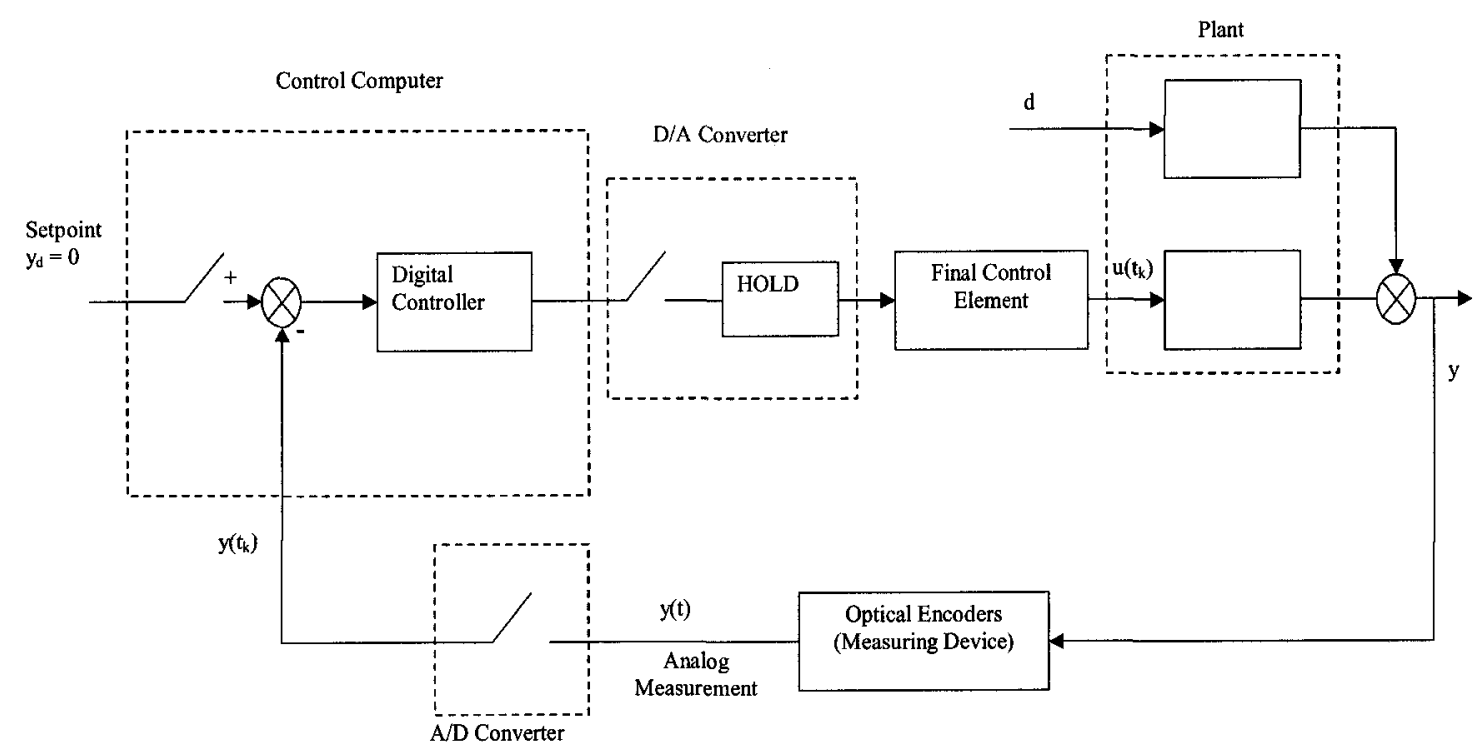

Figure 4.3: Typical Block Diagram of a Computer Control System

additional unwanted consequences that can potentially degrade the performance of the closed loop system. The single most important impact of the discretization process is a time delay associated with a Zero-Order Hold $(\mathrm{ZOH})$ element of the NI DAQ 6036E D/A converter $[53,4]$. The $\mathrm{ZOH}$ keeps the output signal constant in between sampling times and its transfer function is given by

$$
Z O H(j \omega)=T e^{-j \omega T / 2} \operatorname{sinc}(\omega T / 2)
$$

where $T$ denotes the sampling time. It can be observed that the effect of the $\mathrm{ZOH}$ is to introduce a phase shift of $\omega T / 2$ corresponding to a time delay of $T / 2$ seconds as well as to multiply the gain of the plant transfer function by the $T \operatorname{sinc}(\omega T / 2)$ function.

From the above expression, the effect of slow sampling rates can be readily observed: the introduction of additional time delays that can potentially destabilize the closed loop system or reduce the effectiveness of a feedback controller, especially in its ability to cope 
with disturbances of higher frequencies. If a system disturbance occurs within the sampling period (period within which the control signal is held constant by the $\mathrm{ZOH}$ element), the discrete control system will not have a chance to take corrective action. On the other hand, faster sampling rates introduce high frequency noise in the velocity estimation obtained by numerical differentiation of the encoder angular positional data.

To obtain an acceptable, timely, and smooth response (recall that the output of the $\mathrm{ZOH}$ is a staircase-like signal), as well as a satisfactory controller rejection of plant disturbances induced by white noise, Franklin et al [53,485] proposed the following guidelines:

$$
\frac{\omega_{s}}{\omega_{b}} \geq 20
$$

where $\omega_{s}$ denotes the sampling frequency ${ }^{4}$ and $\omega_{b}$ denotes the closed loop bandwidth.

After performing numerous unsuccessful experimental runs that saw the demise of several DC motors, a sampling frequency of $50 \mathrm{~Hz}$ was finally selected. This sampling frequency, which corresponds to a closed loop bandwidth of $2.5 \mathrm{~Hz}$, allowed the computation of a relatively smooth velocity signal devoid of high frequency noise even though the latter was further filtered using a second order low pass filter.

Given the sampling time and discretization method, the continuous system triple is converted to discrete form using the MATLAB command $\mathrm{c} 2 \mathrm{~d}$. The discrete system is given by

$$
\begin{gathered}
\dot{\mathbf{x}}_{\mathbf{a}}(\mathbf{k}+\mathbf{1})=\mathbf{A}_{\mathbf{d}} \mathbf{x}_{\mathbf{a}}(k)+\mathbf{B}_{\mathbf{d}} u(k) \\
\mathbf{y}(k+1)=\mathbf{C x}_{\mathbf{a}}(k)+D u(k)
\end{gathered}
$$


where

$$
\begin{aligned}
\mathbf{A}_{\mathbf{d}} & =\left[\begin{array}{ccccc}
1.00 & -0.02 & 0.00 & 0.00 & 0.00 \\
0.00 & 1.00 & 0.00 & 0.02 & 0.00 \\
0.00 & 0.00 & 0.99 & 0.00 & 0.021 \\
0.00 & 0.28 & -0.04 & 1.00 & 0.01 \\
0.00 & -0.14 & -0.12 & -0.00 & 0.51
\end{array}\right] \quad \mathbf{B}_{\mathbf{d}}=\left[\begin{array}{c}
0 \\
0.00 \\
0.00 \\
0.00 \\
0.32
\end{array}\right] \\
\mathbf{C} & =\left[\begin{array}{lllll}
0 & 1 & 0 & 0 & 0
\end{array}\right] \quad D=0
\end{aligned}
$$

and $k=1,2,3 \ldots$ represents the sampling points, (i.e. the system state $x_{k}$ at time $t_{k}=\mathrm{kT}$ ). The poles of the open loop discrete system are located at 1.00, 1.45, 0.04, 0.69 and 0.97 . The bolded pole outside the unit circle is an indication of an open loop unstable system

\subsection{State Estimation}

The angular position of each link is recorded using an incremental optical encoder. LQR control requires the full state vector be available (i.e. the angular positions and velocities of each link). Angular velocities are obtained by numerically differentiating the angular position of each link. That is at each sampling interval

$$
\begin{aligned}
& x_{4}(k)=\frac{x_{1}(k)-x_{1}(k-1)}{T} \\
& x_{5}(k)=\frac{x_{2}(k)-x_{2}(k-1)}{T}
\end{aligned}
$$

where $\mathrm{T}$ represents the sampling time. The result from the differentiation is passed through a second order Butterworth low pass filter with a cutoff frequency of $2 \mathrm{~Hz}$ to eliminate high frequency noise introduced by numerical differentiation.

\subsection{Controllability and Observability}

Before engaging in the design of an optimal control system, the plant must be controllable and observable. The conditions for observability and controllability govern the existence of 
a solution to the optimal control problem given in Equation (4.4.4). Given these criteria, one can tell if a design solution exist or not for the chosen system parameters and design objectives.

Definition 4.7.1. A plant is said to be controllable if every state variable of the process can be controlled to reach a certain objective in finite time by some unconstrained control $u(t)[32,273]$.

For the system described by the state equations (4.5.3) to be controllable, it is necessary and sufficient that the following $n x n$ controllability matrix be full rank:

$$
S_{c}=\left[\begin{array}{llll}
B_{d} & A_{d} B_{d} & A_{d}^{2} B_{d} & A_{d}^{3} B_{d}
\end{array}\right]
$$

The practical significance of the controllability condition is the ability to place the closed loop poles of the system arbitrarily.

The concept of observability relates to the estimation of state variables from output variables that are measurable. One practical problem associated with the implementation of state feedback control is the physical inaccessibility of certain state variables-those that do not influence the system output as defined by observability condition. Where such situations exists, it may be necessary to design an observer that will estimate these nonaccessible state vectors from the system output vector. The condition that such an observer can be designed for the system is called the observability of the system.

Definition 4.7.2. A plant is said to be observable if every state variable of the system affects the output(s) [32, 280].

For the system described by the state equations (4.5.3) to be observable, it is necessary and sufficient that the following $n x n$ observability matrix be full rank

$$
S_{o}=\left[\begin{array}{llll}
C & C A_{d} & C A_{d}^{2} & C A_{d}^{3}
\end{array}\right]^{T}
$$


It can be confirmed that the controllability matrix $S_{c}$ has a rank of 5 , indicating that the augmented discretized system is controllable. The observability matrix $S_{o}$, on the other hand, has a rank of 4 , indicating that not all five states can be estimated from the output measurement. Given that satisfactory estimation of the nonmeasurable states are obtained using numerical differentiation and integration, the requirement for observability can be relaxed.

\subsection{Simulation Results}

The gains for the LQR control algorithm given in Equation (4.4.5) are obtained using the MATLAB command dlqr. The state and control penalties, $\mathbf{Q}$ and $R$ respectively, required for the solution of the LQR problem are selected using trial and error based on the following guidelines:

1. The state and penalty matrices should be selected such that, control effort is more penalized than state excursions from the desired equilibrium.

2. The state penalty position diagonals, $Q(2,2)$ and $Q(3,3)$ which affect the controller positional gains, should be selected to penalize excursions of the angular displacements from the equilibrium to a greater extent. In order words, the positional components of the control law should contribute much more than the velocity components. Therefore, higher penalties should be assigned for excursions of $x_{1}$ and $x_{2}$ from the equilibrium, resulting in larger positional gains.

3. The state penalty diagonals, which dictate the controller velocity gains $Q(4,4)$ and $Q(5,5)$ are chosen such that high frequency noise within the velocity computation, if present, is not amplified for this to cause system instability. 
By carrying out numerous simulations based on the above guidelines the state and control penalties were finally set to

$$
\mathbf{Q}=\left[\begin{array}{ccccc}
1 & 0 & 0 & 0 & 0 \\
0 & 300 & 0 & 0 & 0 \\
0 & 0 & 200 & 0 & 0 \\
0 & 0 & 0 & 1 & 0 \\
0 & 0 & 0 & 0 & 1
\end{array}\right] \quad R=68
$$

resulting in the following controller gains:

$$
\mathbf{K}_{L Q R}=\left[\begin{array}{lllll}
0.11 & -99.79 & 13.82 & -24.63 & -0.03
\end{array}\right]
$$

With these gains, the discrete closed loop poles are located at $0.44,0.99,0.98,0.92$, and 0.9339. The closed loop poles are very close to the unit circle, suggesting room for improvement of controller performance. However, it should be noted that these closed loop poles correspond to a lower bound of controller gains that would stabilize the system based on the selected operating parameters.

The efficacy of the controller in rejecting four types of disturbances will be considered. The first type of disturbance is a constant voltage applied at the servo amplifier input. Such a disturbance could result from a small voltage offset in the servo amplifier used to drive the DC motor. The second type of disturbance considered is a periodic voltage applied to the input of the servo amplifier. A disturbance comprising two opposing pulses, $1 \mathrm{sec}$ in width, spaced $20 \mathrm{sec}$ apart, is also considered. Each pulse has an absolute magnitude of $1 \mathrm{~V}$. These disturbances are applied with all system states initially set to zero. Finally, the system states are subjected to non-zero initial values and the controller behavior is monitored. To add some degree of reality, nonlinearities, such as deadband, white noise in the control input, and a ZOH element, are all included in the Simulink model. A representation of the Simulink model used for simulation of the closed loop system is given in Figure 4.4. 
Angular velocities estimates obtained by differentiating the positional vectors and applying the result through a first order low pass filter with a cutoff frequency of $2 \mathrm{~Hz}$ are generated within the block labelled 'State Estimate'. A frequency cutoff of $2 \mathrm{~Hz}$ is chosen, given that signals with greater frequencies excite high frequency unmodelled dynamics. The original nonlinear equations describing the dynamics of the robot are modelled in the block labelled Plants.

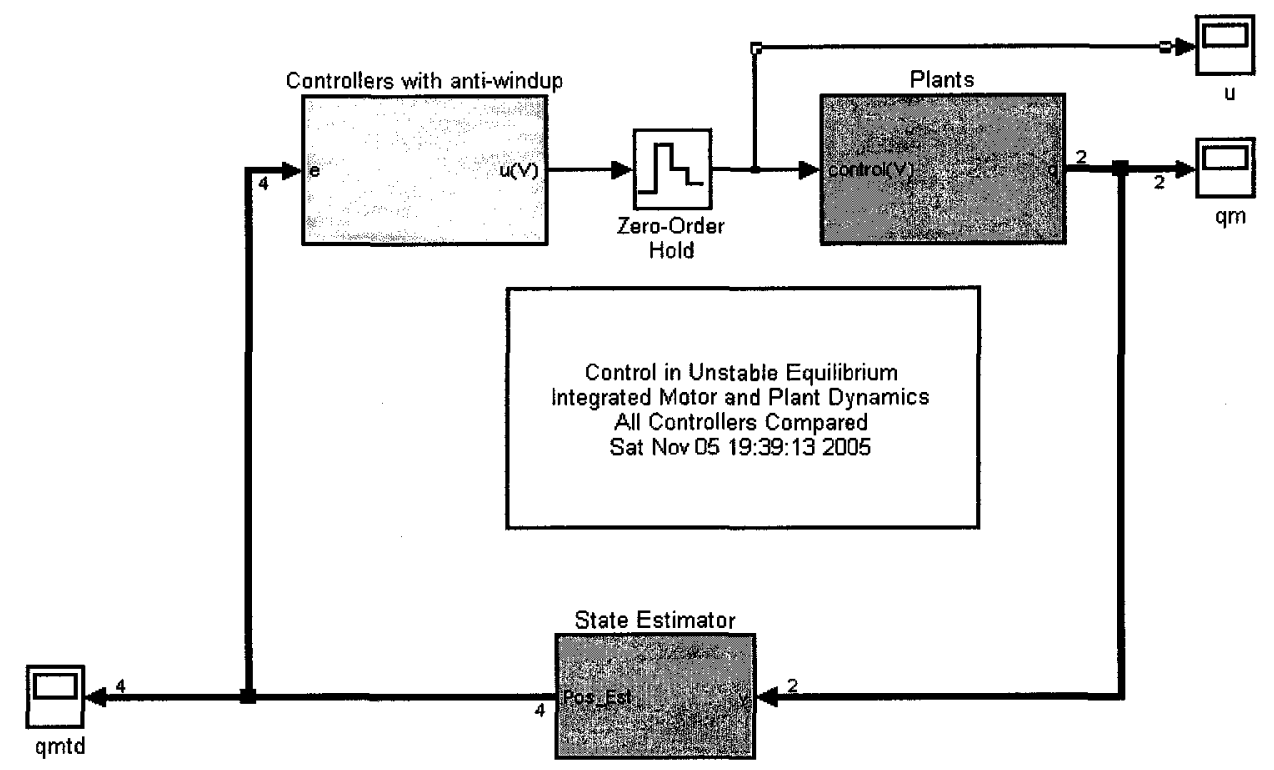

Figure 4.4: Simulink Model of Plant and Controller

\subsubsection{Constant Voltage Disturbance}

The constant disturbance tested comprises a constant voltage signal applied persistently to the servo amplifier driving the robot's DC motor. This disturbance, C, enters the system through the same channel as the control input (i.e.

$$
V_{a}=u+C
$$


where $V_{a}$ is the actual voltage applied to the servo amplifier, $u$ is the computed control voltage, and $C$ is the constant disturbance). A value of $\mathrm{C}=0.1 \mathrm{~V}$ was used in the simulation. It can be observed from Figure 4.5 that the constant voltage disturbance forces the LQR
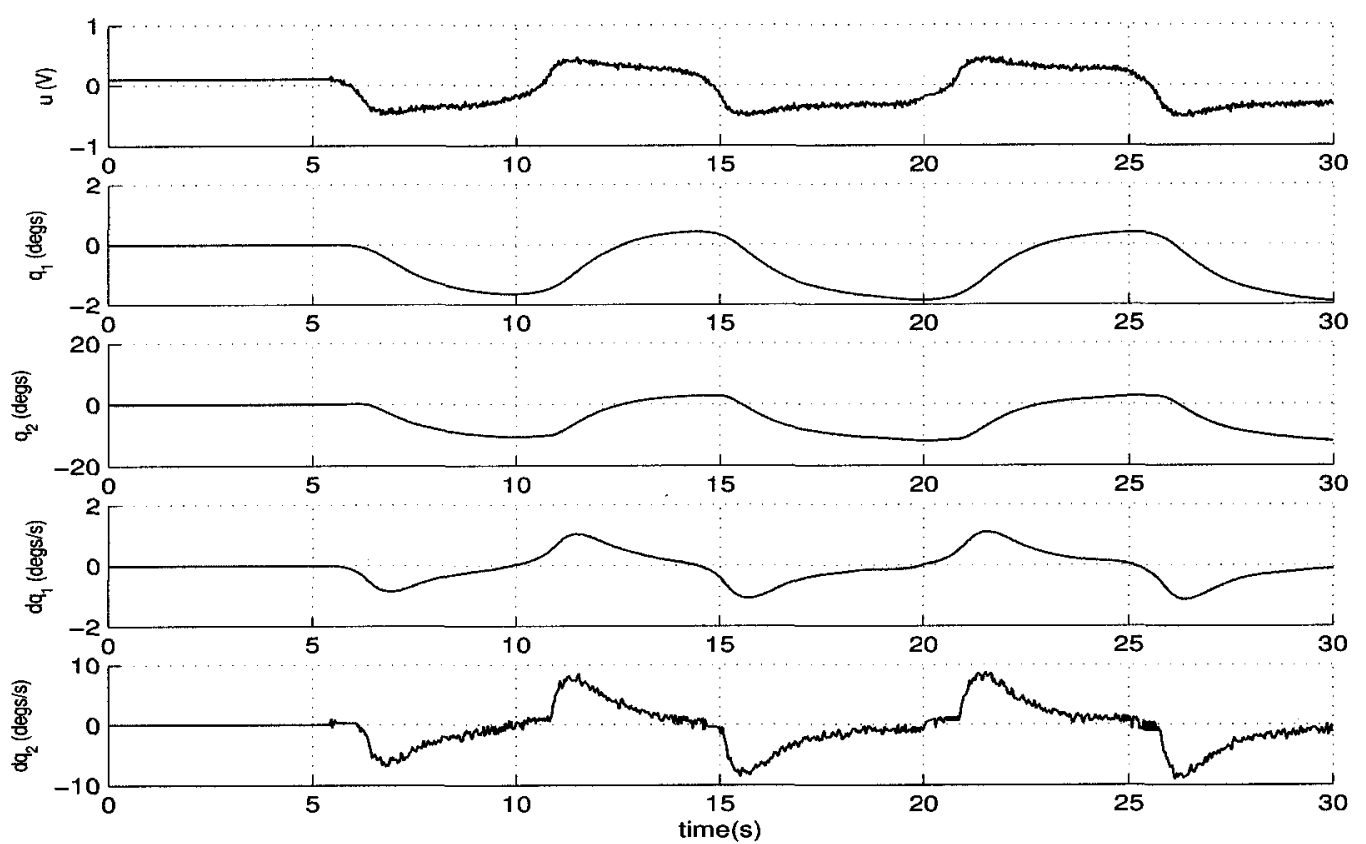

Figure 4.5: Simulation Result of LQR Control in Response to a Constant Disturbance

to balance the robot about an equilibrium other that the desired. This suggests that the integral gain needs to be further tuned.

\subsubsection{Periodic Voltage Disturbance}

The periodic disturbance tested comprises a $1 \mathrm{~Hz}$ sinusoidal voltage signal with an amplitude of $1 \mathrm{~V}$. As before, this disturbance is persistently applied through the same channel as the control input (i.e.

$$
V_{a}=u+2 \sin (2 \pi t)
$$



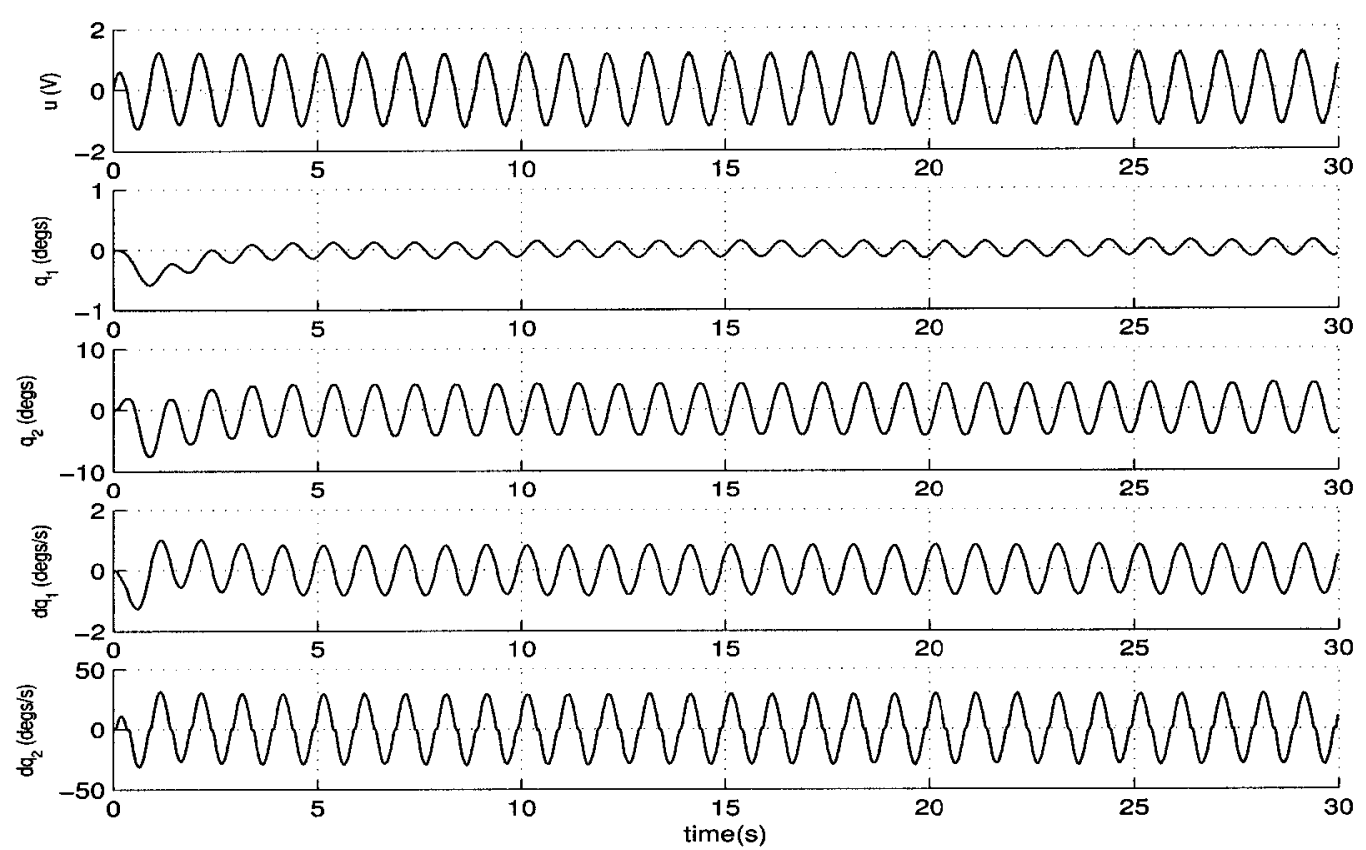

Figure 4.6: Simulation Result of LQR Control in Response to a Sinusoidal Disturbance

where $V_{a}$ is the actual voltage applied to the servo amplifier and $u$ is the computed control voltage). The simulation result, presented in Figure 4.6, reveals a balanced system, even though the disturbance is hardly attenuated.

\subsubsection{Pulsed Voltage Disturbance}

A disturbance comprising two opposing voltage pulses, $1 \mathrm{sec}$ wide and $20 \mathrm{sec}$ apart, each with an absolute magnitude of $1 \mathrm{~V}$ is also considered. In contrast to the two previous cases, this disturbance is momentarily applied with the system in open loop and control is handed over to the LQR until the next pulse occurs, after which the cycle repeats itself. Figure 4.7 reveals an overall satisfactory response of the LQR. 


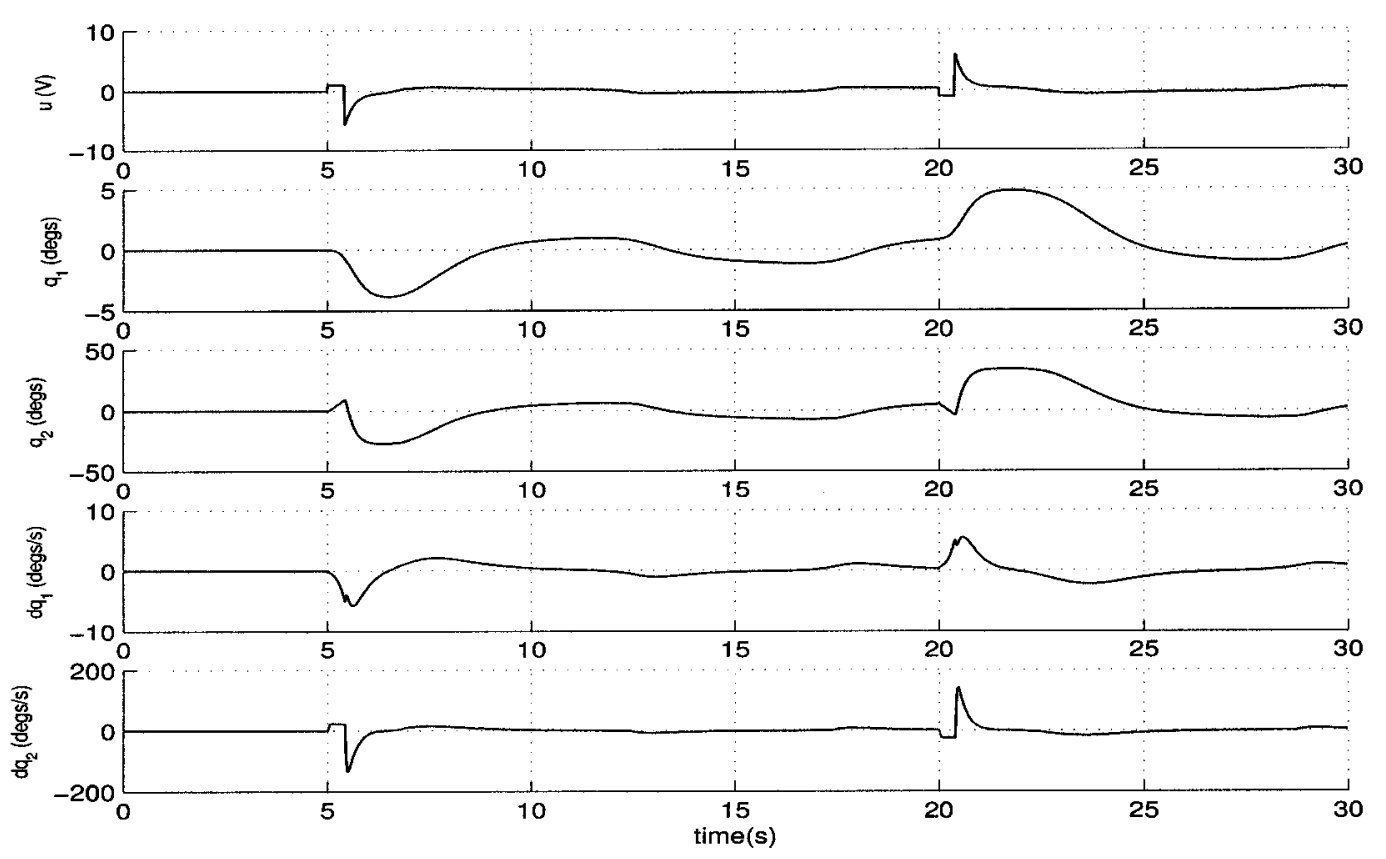

Figure 4.7: Simulation Result of LQR in Response to 2 pulses

\subsubsection{Initial Displacement from Equilibrium}

In the aforementioned scenarios, all state variables are initially set to zero. To excite the system with a different form of disturbance, the state variables are initially set as follow: $q_{1}=2.5^{\circ}, q_{2}=4^{\circ}, \dot{q}_{1}=3^{\circ} /$ sec and $\dot{q}_{2}=-5^{\circ} /$ sec. The arbitrary initial velocities are chosen such that the first link is moving farther away from the equilibrium position (counterclockwise) while the second link is moving clockwise in relation to the first link. Note that this motion causes an imbalance of the system's overall center of gravity, given that the center of gravity of both links are moving towards the left simultaneously. Figure 4.8 demonstrates the effectiveness of the LQR in returning the links to the equilibrium position. Notice the rapid initial change of direction forced on link 2 by the LQR so as to counterbalance the displaced overall center of gravity. To appreciate the effectiveness of the LQR controller, on the other hand, the response of the open loop system to a sinusoidal 


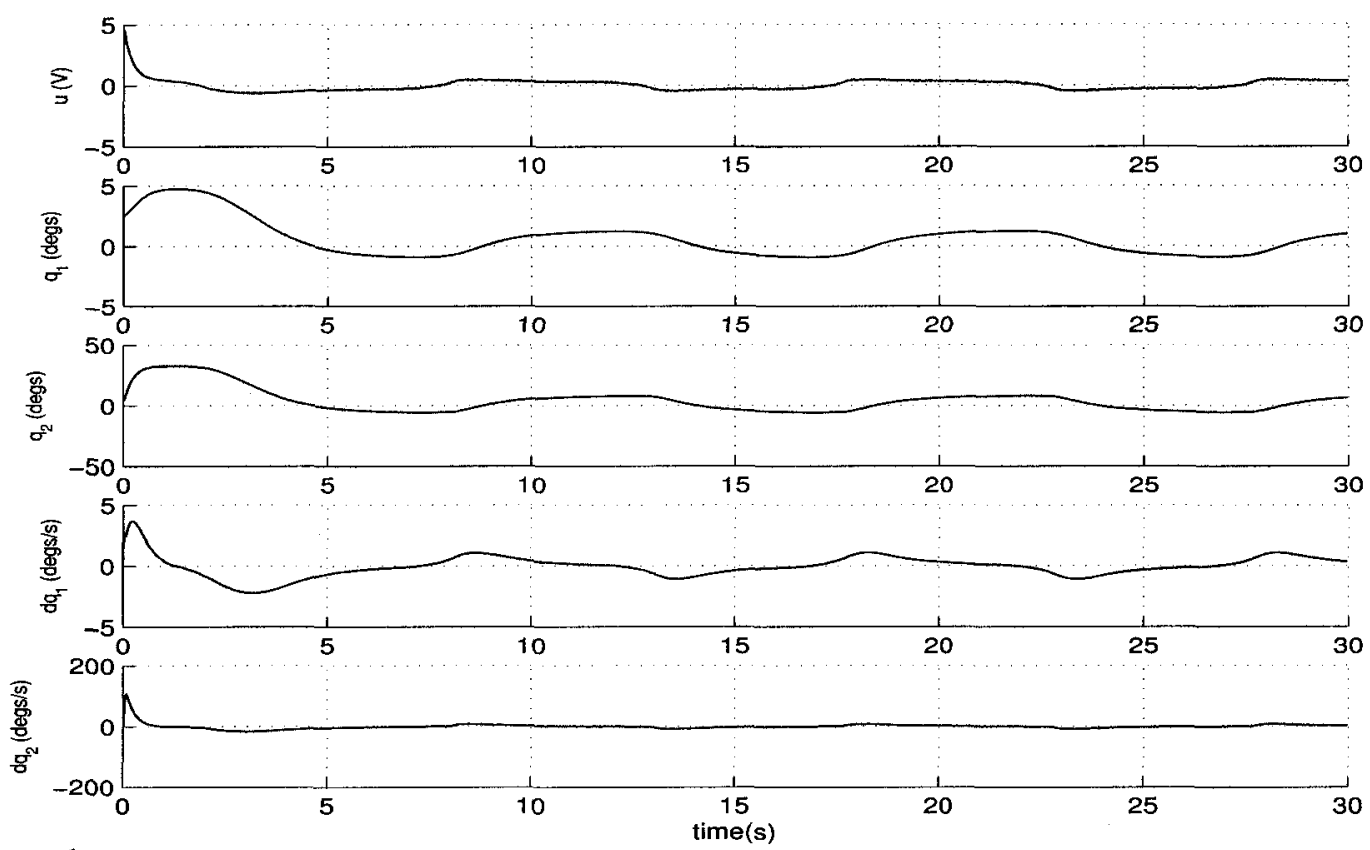

Figure 4.8: Simulation Result of LQR in response to non-zero Initial Conditions
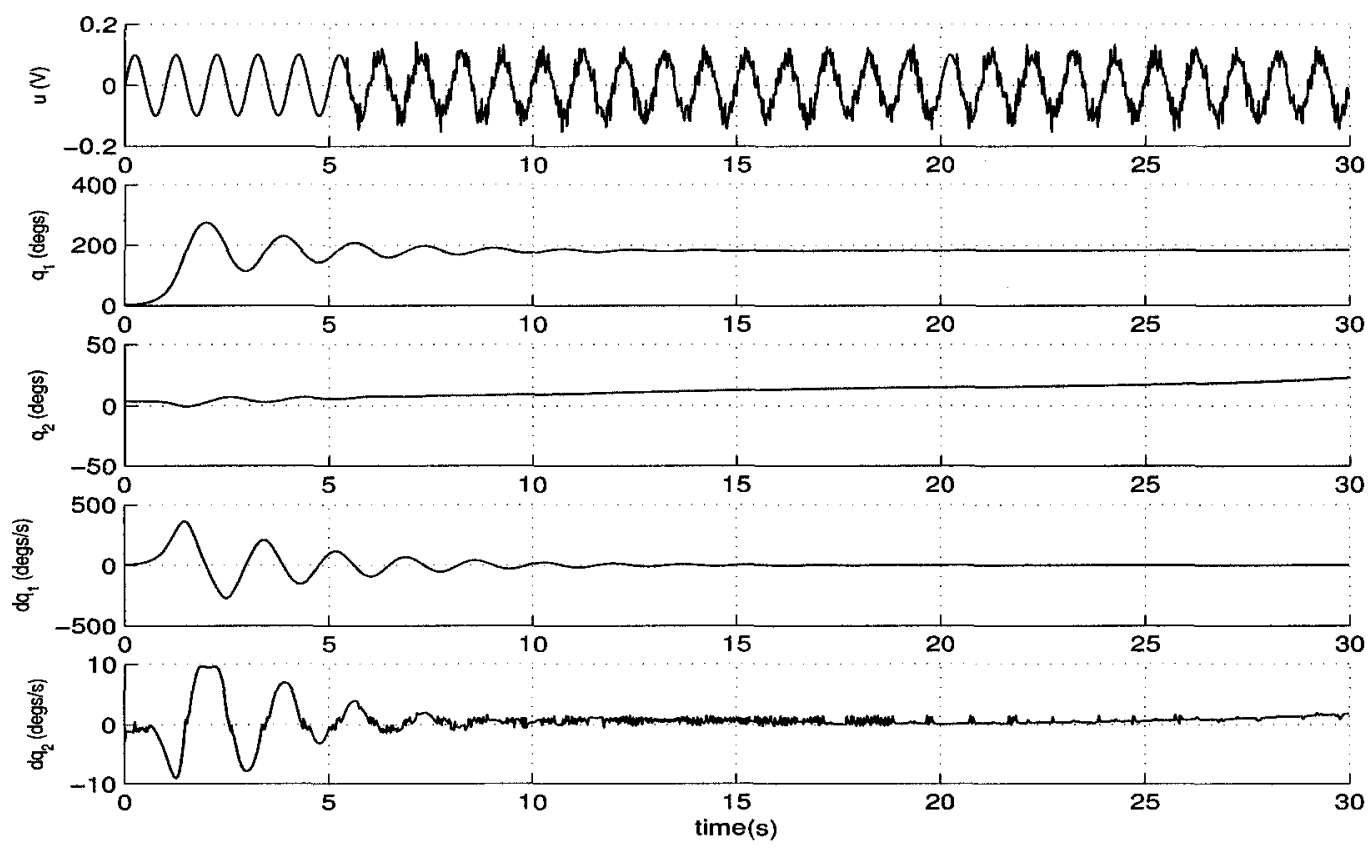

Figure 4.9: Open Loop System Response to Sinusoidal Disturbance 
disturbance, with all state variables set to zero, is given in Figure 4.9. Observe that the system quickly 'falls' down to the stable inverted position.

\subsection{Experimental Results}

To validate the performance of the controller, the real system is subjected to the pulsed disturbance introduced in Section 4.8 .3 with the initial state variables reading $q_{1}=q_{2}=$ $\dot{q}_{1}=\dot{q}_{2}=0$. At this stage of the exercise, the sampling time of the closed loop system, as discussed in Section 4.5 , is fixed at $0.02 \mathrm{~s}$. Figure 4.10 illustrates the effectiveness of the controller in rejecting this type of disturbance as well as in eliminating the steady state error.

To verify controller consistency or repeatability of behaviour, several
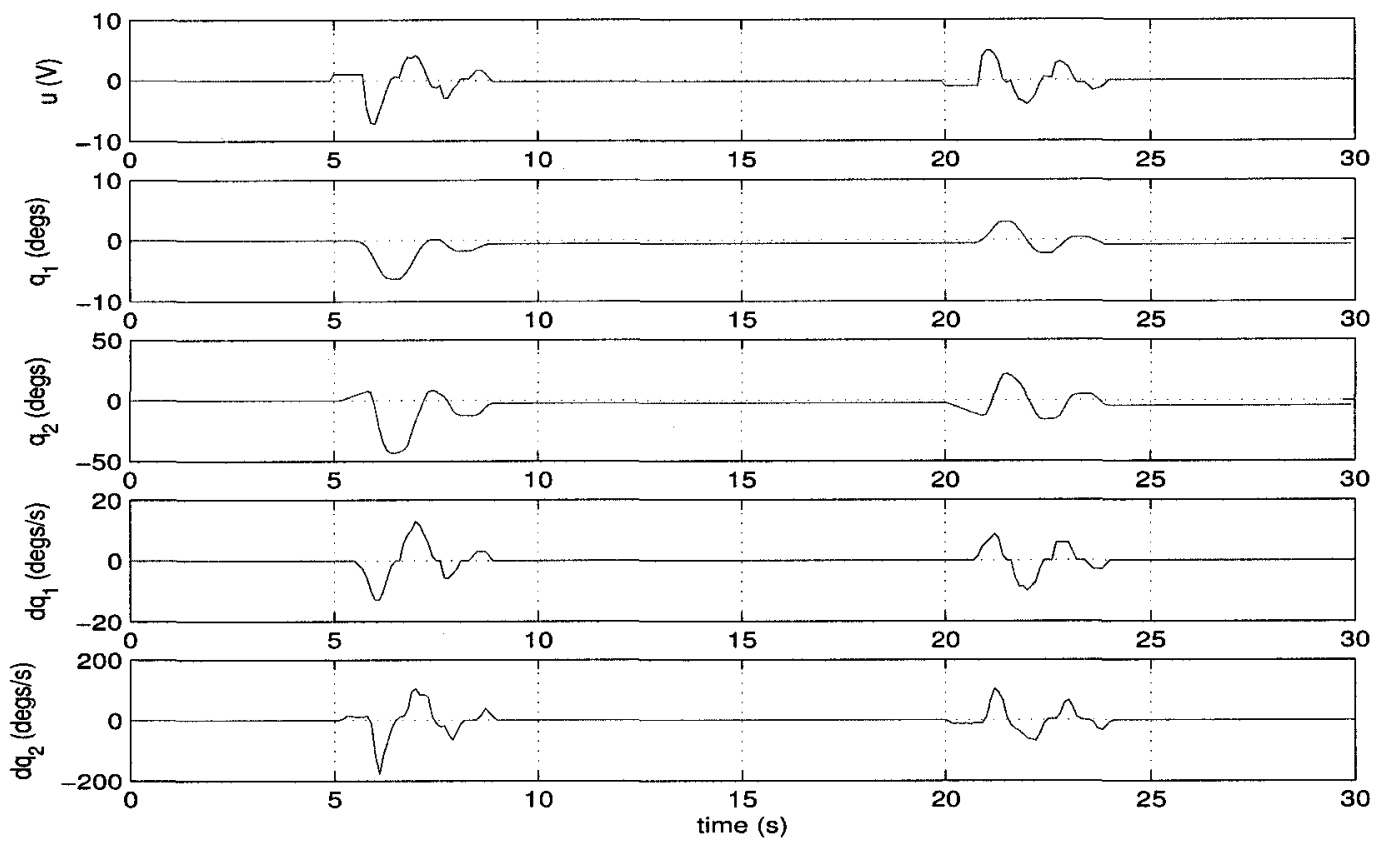

Figure 4.10: Experimental Result of LQR in Response to 2 Pulses

experimental runs were obtained. The results are displayed in Figures 4.11 and 4.12. Even 


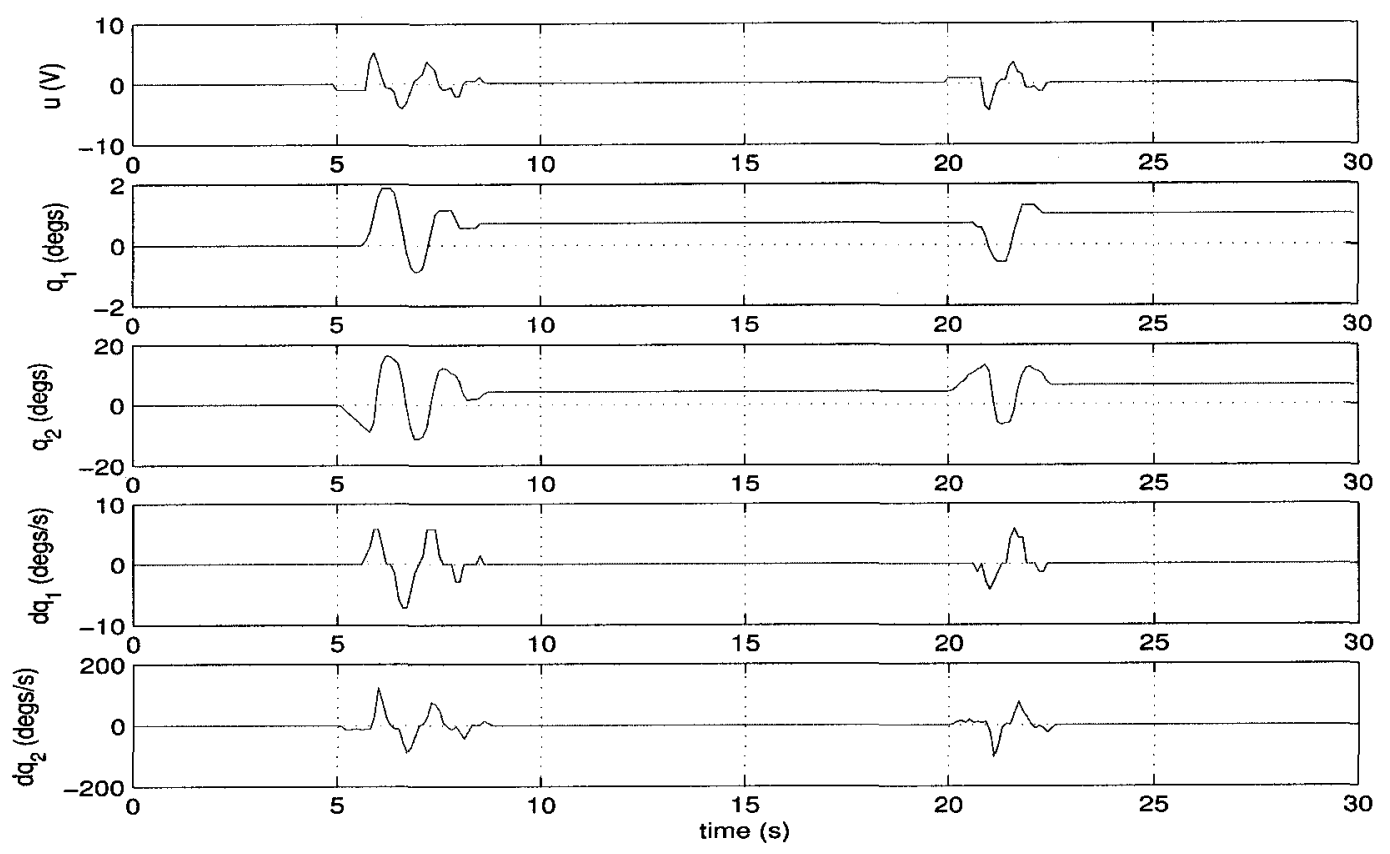

Figure 4.11: Second Experimental Result of LQR in Response to 2 pulses

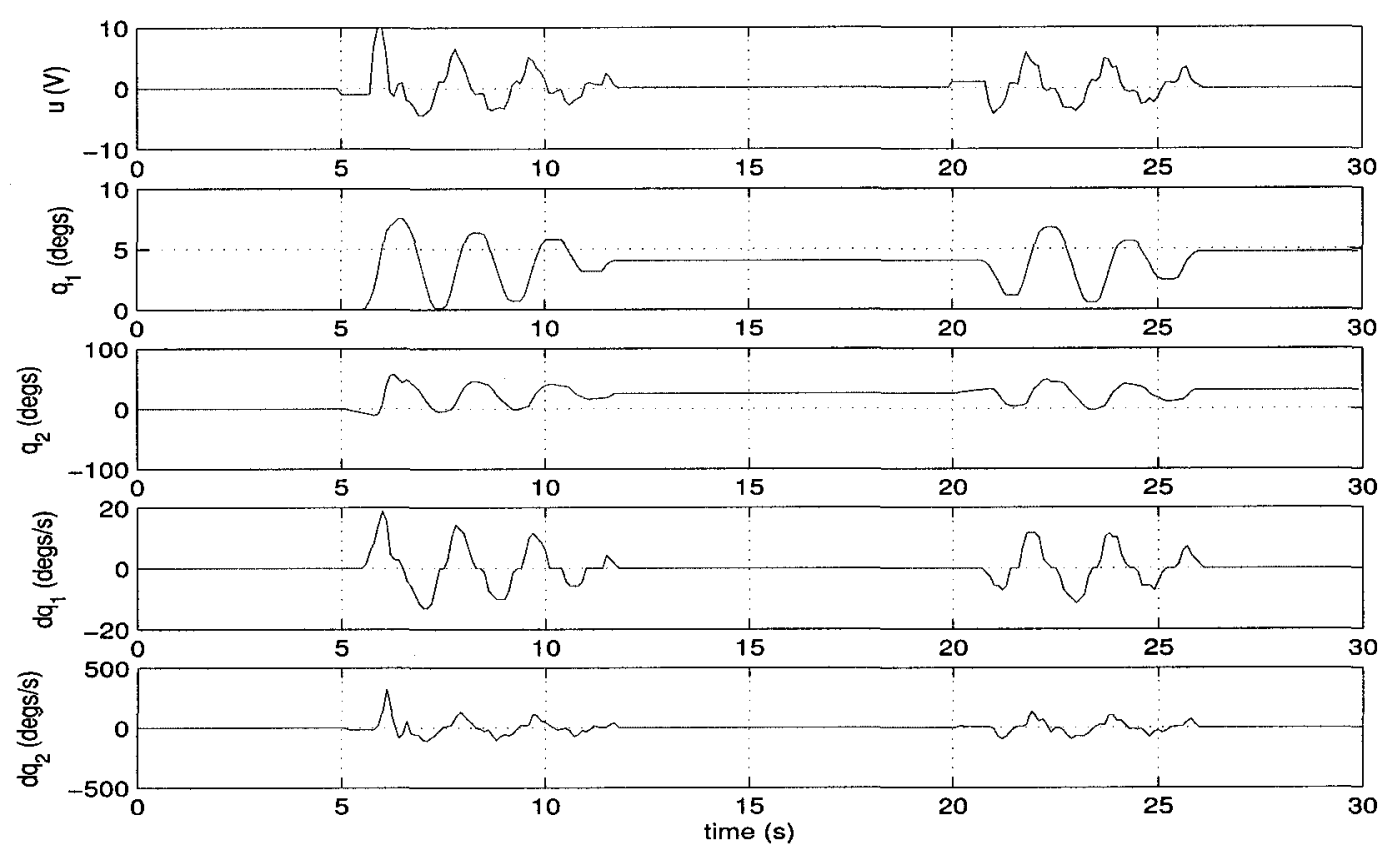

Figure 4.12: Third Experimental Result of LQR in Response to 2 pulses 

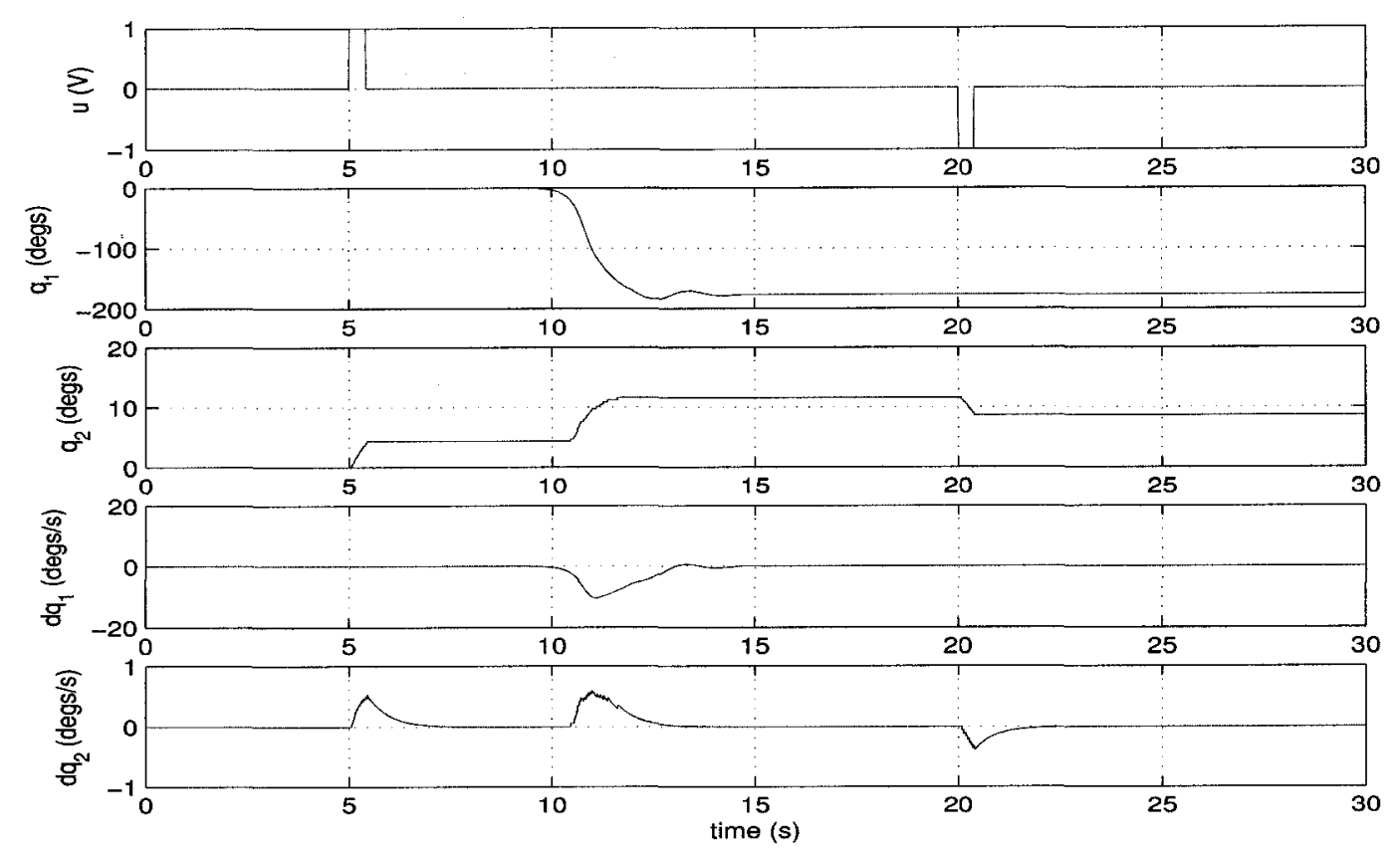

Figure 4.13: Real System Subjected to a Single Pulse in Open Loop

though the controller successfully stabilizes the system on every occasion, there appears to be inconsistencies in the elimination of steady state error as illustrated in Figures 4.11 and 4.12. Furthermore, the presence of overshoot in the system response is eminent, although, in all fairness, no strict performance objectives were specified. These results suggest that the performance of the controller needs to be improved to achieve the control and performance objectives of the particular application.

Nonetheless, one can appreciate the effectiveness of the controller, by subjecting the open loop system to a pulse disturbance and observing how fast it 'falls' to the stable equilibrium. This results are presented in Figure 4.13. Note how quickly the system becomes unstable eventually falling to the stable equilibrium or the upside-down position when link 2 is subjected to a disturbance. 


\subsection{Mechanical Power vs. Span Angle $\phi$}

The theoretical effect of changes in the body span angle, $\phi$, on the control effort required for stability was given in Section 2.7. It was concluded, solely by referring to the literature, that changes in $\phi$ transform the system from minimum to non-minimum phase, consequently affecting the control effort required to maintain stability.

It must be noted, however, that the LQR control system does not alter the position of transfer function zeros of the 2 DOF robot. Instead, the control system relocates the open loop transfer function poles to desired positions explicitly defined by a set of control objectives. Control effort, therefore, is expended in moving open loop transfer function poles from their initial to the desired position.

Referring to Figure 3.15, one can observe that open loop transfer function poles locations, are not significantly different for different values of $\phi$. More noticeable, though, is changes in transfer function zero locations for different values of $\phi$. Therefore, it should be fairly accurate to attribute changes in control gains to a given reference set of closed loop poles to changes in $\phi$.

To verify the above postulation, a closed loop simulation of the linearized system was carried out for several values of $\phi$. To allow for a fair comparison, a desired set of closed loop poles is initially specified and controller gains for each body configuration are obtained using the MATLAB command acker. The mechanical power is computed using the expression

$$
P=\int_{t=0}^{t_{n}} u d t
$$

Figure 4.14 illustrates changes in the mechanical power consumption for various values of $\phi$ when each system is subjected to the same initial disturbance. It can be seen that mechanical power savings can be realized by carefully selecting $\phi$. 


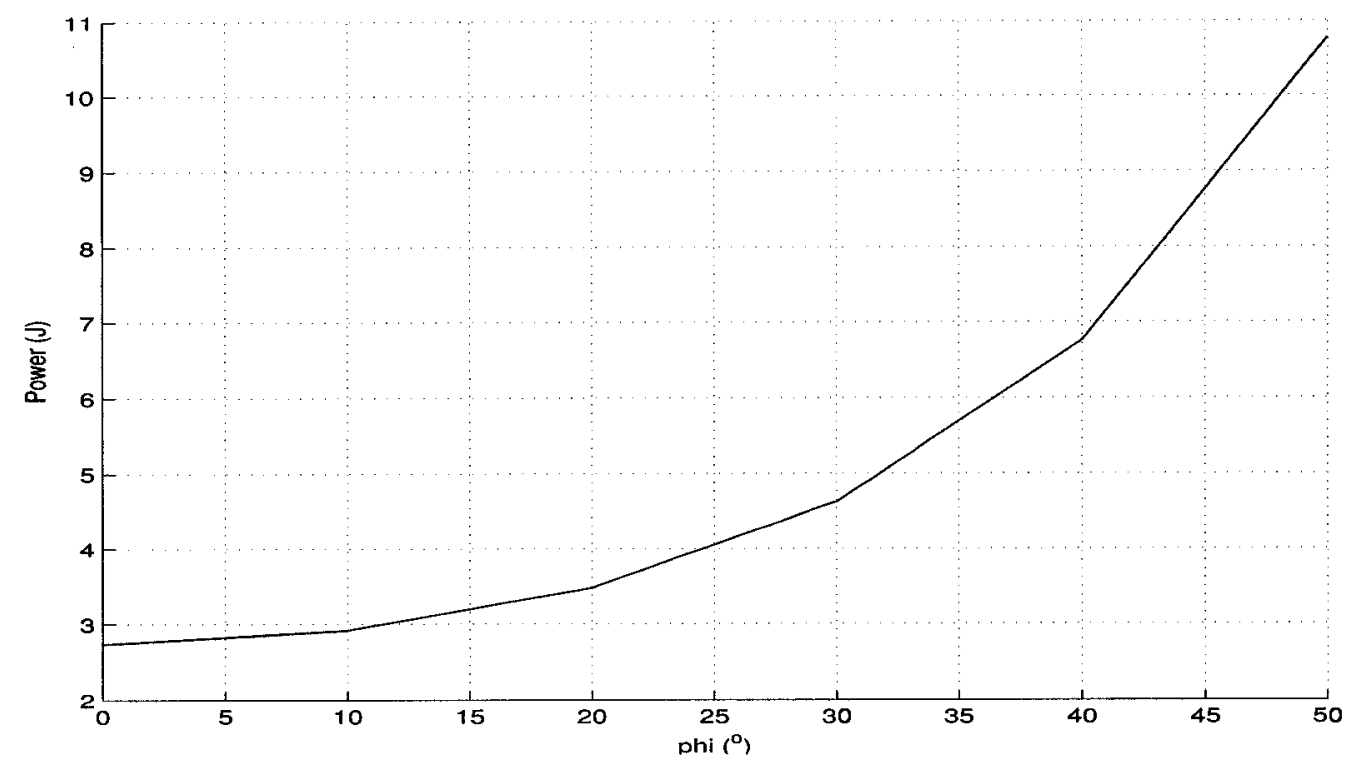

Figure 4.14: Mechanical Power vs. $\phi$

\subsection{Conclusion}

In this chapter, LQR with Integral action was successfully used to balance a double inverted pendulum about its unstable equilibrium. The experimental setup was carefully designed to mimic the stance phase of a hopping cycle, characteristic of one-legged 2 DOF hopping robots. Simulation results demonstrated the effectiveness with which the LQR rejected different types of system disturbances. Numerous experimental results, with the system subjected to a pulsed disturbance, further confirmed that the controller will consistently reject disturbances that fall within its operational range. Given the absence of a set of performance specifications, however, conservative controller gains were selected. These represent the lowest set of gains that stabilize the model in simulation, although, the response of the real plant is slightly more aggressive.

The control system exhibits overshoot, and its ability to completely eliminate steady 
state error, which can result from an offset in the operational amplifier, for instance, is questionable. In this regard, there is room for improvement in terms of controller performance.

One main objective of this exercise is to minimize the power consumed by the system while not compromising the performance objectives. Although the smallest control gains that guarantee stability of the simulated closed loop system were chosen, the overall power consumption of the system, with respect to existing control methods has not been assessed. Furthermore, an experimental verification of the effect of control effort for changes in $\phi$ would have to be confirmed. These exercises could serve as a good starting point for further work in this research.

One fundamental limitation of the LQR is its limited operation range. In actual implementation, it would be desirable for the control system to be able to withstand larger operational ranges, even in the presence of modelling uncertainty. In the next chapter, a nonlinear control strategy is synthesized in an attempt to increase the operation range of the robot control system. 


\section{Chapter 5}

\section{Feedback Linearizing Control}

\subsection{Introduction}

The mathematical representation of a process plays a dominant role in model-based control design. The Linear Quadratic Regulator synthesized in the previous chapter is a practical example of a control system based on the mathematical model of the plant. The Feedforward controller [52, 571], Smith Predictor [52, 605], Inverse-Response Compensator [52, 613] and the Model Predictive Controller (MPC) [52, 991], just to name a few, are several examples of practical model-based controllers. Amongst the fundamental problems of system analysis, model-based control solves the "Process Control" problem summarized as follows:

Given a desired output behavior and the process model, derive a controller that would enforce the specified output behavior.

The performance of the derived controller in meeting the control objectives is, therefore, constrained by how accurately the process model describes the behavior of the real process.

Virtually, all real world systems are nonlinear and certain physical phenomena cannot exactly be described using mathematical relationships. A typical strategy in such a case is to linearize the original system dynamics around a desired operating point. Such approaches, 
which typically result in linear controllers such as the LQR synthesized in the previous chapter, have been successful in industry. This is because most physical processes take on approximately linear behavior as they approach steady state [52, 625].

Linear controllers, thus, perform reasonably well for small excursions from steady state. For instance, the LQR synthesized in the previous chapter performs satisfactorily for angular displacements less than $8^{\circ}$. For large excursions from steady state, however, or when modelling uncertainty exists, performance of the linear control system deteriorates. In this case, more effective alternatives must be considered.

Nonlinear control systems, on the other hand, explicitly recognize uncertainty or nonlinearities in the process model. As a result, these control strategies allow larger operation ranges and often offer larger tolerances to plant or modelling uncertainties. So, when the nature of the process demands tight performance specifications, large excursions from steady state, and robustness to modelling uncertainty, nonlinear control is the method of choice. The noticeable increase in academic and industrial research in the area of nonlinear control has been spurred in part by the advantages nonlinear controllers offer over their linear counterparts, although recent advances and availability of low-cost and high speed microprocessors have also played a major role.

\subsection{Overview}

In this chapter, the control of balance in the one-legged robot is attempted using Feedback Linearization (FL), a nonlinear control strategy. Feedback Linearization has successfully been used to address some practical control problems in systems such as high performance aircraft [54], industrial drives [55], and spark ignition engines [56]. Basically, it involves transforming the original nonlinear dynamics of a system into an equivalent linear model via 
exact state transformation and feedback. In contrast to Jacobian linearization used in LQR control design, for instance, where the effects of nonlinearities in the plant model are ignored, Feedback linearization attempts to deal with the nonlinearities via state transformation and feedback. As a result, one naturally should expect better performance in terms of larger operation range. However, due to the fact that exact cancellation of the system nonlinearities requires an accurate model, Feedback Linearization controllers are potentially sensitive to parametric and modelling uncertainty.

The remainder of this chapter presents the synthesis of a feedback linearizing controller to maintain an upright posture on a one-legged monopod. Control objectives and the mathematical formulation of the plant are referenced from the previous chapter.

\subsection{Controller Design}

The first step in designing a control system for any given physical plant is to derive a meaningful model of the plant (i.e. one that captures the dominant dynamics around the operation range of interest). The basic idea behind feedback linearization is to perform a coordinate transformation on the original system dynamics via feedback such that, with respect to the new coordinates, the model is linear. Within this coordinate system, linear control techniques can then be applied. Feedback linearization applies only to fully actuated systems. However, input-state linearization is a variant that shares similar properties with feedback linearization and can be applied to underactuated systems.

Consider the original system dynamics

$$
\left[\begin{array}{l}
\ddot{q}_{1} \\
\ddot{q}_{2}
\end{array}\right]=\left[\begin{array}{l}
f_{3}\left(q_{1}, q_{2}, \dot{q}_{1}, \dot{q}_{2}\right)+g_{3}\left(q_{2}\right) u \\
f_{4}\left(q_{1}, q_{2}, \dot{q}_{1}, \dot{q}_{2}\right)+g_{4}\left(q_{2}\right) u
\end{array}\right]
$$


where

$$
f_{3}=\frac{\left(\begin{array}{l}
\left(m_{3} m_{12} s_{2} \dot{q}_{1}^{2}-m_{3} m_{14} \dot{q}_{2}+m_{1} c_{2} m_{12} s_{2} \dot{q}_{1}^{2}+m_{1} c_{2} m_{14} \dot{q}_{2}+m_{13} \dot{q}_{1} f_{r 1}\right. \\
+2 m_{13} \dot{q}_{1} m_{1} s_{2} \dot{q}_{2}+m_{13} m_{1} s_{2} \dot{q}_{2}^{2}-\frac{m_{3}\left(f_{c 2}+m_{10}\right) \operatorname{sgn}\left(\dot{q}_{2}\right)}{m_{7}}-m_{13} m_{5} s_{1}+m_{13} m_{6} s_{12} \\
\left.+\frac{m_{1} c_{2}\left(f_{c 2}+m_{10}\right) \operatorname{sgn}\left(\dot{q}_{2}\right)}{m_{7}}-m_{3} m_{16} s_{12}+m_{1} c_{2} m_{16} s_{12}+m_{13}\left(f_{c 1}\right) \operatorname{sgn}\left(\dot{q}_{1}\right)\right)
\end{array}\right)}{\left(m_{11} m_{3}-m_{11} m_{1} c_{2}+m_{12} c_{2} m_{3}-m_{12} c_{2}\right)^{2} m_{1}-m_{13} m_{2}+2 m_{13} m_{1} c_{2}}
$$

$f_{4}=\frac{\left(\begin{array}{l}\left(-\left(m_{1} s_{1} \dot{q}_{2}+f r 1\right) \dot{q}_{1}-m_{1} s_{2}\left(\dot{q}_{1}+\dot{q}_{2}\right) \dot{q}_{2}+m_{5} s_{1}-m_{6} s_{12}-\frac{f_{c 1} \operatorname{sgn}\left(\dot{q}_{1}\right)}{m_{3}-m_{1} c_{2}}\right. \\ -\left(m_{2}-2 m_{1} c_{2}\right)\left(m_{3} m_{12} s_{2} \dot{q}_{1}^{2}-m_{3} m_{14} \dot{q}_{2}+m_{1} c_{2} m_{12} s_{2} \dot{q}_{1}^{2}+m_{1} c_{2} m_{14} \dot{q}_{2}\right. \\ +2 m_{13} \dot{q}_{1} m_{1} s_{2} \dot{q}_{2}+m_{13} \dot{q}_{1} f_{r 1}+m_{13} m_{1} s_{2} \dot{q}_{2}^{2}-\frac{m_{3}\left(f_{c 2}+m_{10}\right) \operatorname{sgn}\left(\dot{q}_{2}\right)}{m_{7}}-m_{13} m_{5} s_{1} \\ \left.\left.-m_{3} m_{16} s_{12}+\frac{m_{1} c_{2}\left(f_{c 2}+m 10\right) \operatorname{sgn}\left(\dot{q}_{2}\right)}{m_{7}}+m_{1} c_{2} m_{16} s_{12}+m_{13} m_{6} s_{12}+m_{13} f_{c 1} \operatorname{sgn}\left(\dot{q}_{1}\right)\right)\right)\end{array}\right)}{\left(m_{11} m_{3}-m_{11} m_{1} c_{2}-m_{12} c_{2} m_{3}-m_{12} c_{2}\right)^{2} m_{1}-m_{13} m_{2}+2 m_{13} m_{1} c_{2}}$

$g_{3}=\frac{m_{3}-m_{1} c_{2}}{\left(m_{11} m_{3}-m_{11} m_{1} c_{2}-m_{12} c_{2} m_{3}-m_{12} c_{2}\right)^{2} m_{1}-m_{13} m_{2}+2 m_{13} m_{1} c_{2}}$

$g_{4}=\frac{-m_{2}+2 m_{1} c_{2}}{\left(m_{11} m_{3}-m_{11} m_{1} c_{2}-m_{12} c_{2} m_{3}-m_{12} c_{2}\right)^{2} m_{1}-m_{13} m_{2}+2 m_{13} m_{1} c_{2}}$

and the constants, $m_{i}, i \in[1: 16]$ were defined in Chapter 2 and $u$ is the computed control input.

We want to find a state transformation $z=T(\mathbf{x})$ and input transformation $u=\varrho(\mathbf{x})+$ $\xi(\mathbf{x}) v$ such that the original system dynamics is transformed into an equivalent linear timeinvariant dynamies of the form

$$
\dot{z}=A z+B v
$$

with

$$
A=\left[\begin{array}{llll}
0 & 1 & 0 & 0 \\
0 & 0 & 1 & 0 \\
0 & 0 & 0 & 1 \\
0 & 0 & 0 & 0
\end{array}\right] \quad B=\left[\begin{array}{l}
0 \\
0 \\
0 \\
1
\end{array}\right]
$$

For the original dynamics (5.3.1) to be Feedback Linearizable, there must exist a region $\Re$ such that the following conditions hold: 
1. The matrix

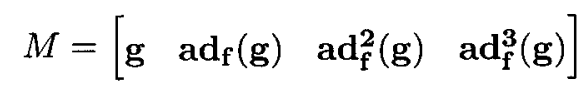

is full rank where the term $\operatorname{ad}_{\mathbf{f}}^{\mathbf{k}}(\mathbf{g})$ denotes the iterative Lie Bracket $\left[\mathbf{f}, \operatorname{ad}_{\mathbf{f}}^{\mathbf{k}-\mathbf{1}}(\mathbf{g})\right.$ ] where the Lie Bracket of $\mathbf{f}$ and $\mathbf{g}$ is defined as $[\mathbf{f}, \mathbf{g}]=\frac{\partial \mathbf{g}_{\mathbf{f}}}{\partial \mathbf{x}}-\frac{\partial \mathbf{f}}{\partial \mathbf{x}} \mathbf{g}$. For simplicity of notation, we will represent this control matrix as $M=\left[\begin{array}{llll}w_{1} & w_{2} & w_{3} & w_{4}\end{array}\right]$. The above condition is mathematically equivalent to:

$$
\operatorname{det}(M) \neq 0
$$

2. The set of vectors $\left\{\mathbf{g}, \mathbf{a d}_{\mathbf{f}}(\mathbf{g}), \mathbf{a d}_{\mathbf{f}}^{\mathbf{2}}(\mathbf{g})\right\}$ is involutive in $\Re$. Mathematically, this can be represented as

$$
\left[w_{1}, w_{2}\right]=\left[w_{1}, w_{3}\right]=\left[w_{2}, w_{3}\right]=0
$$

As mentioned in the Abstract of this thesis, one consequence of setting the angle between the arms of link 2 to $\phi_{o p t}$ is that it eliminates the zeros of the system transfer function. In the absence of these non-minimum phase zeros, the system also appears to be inputstate linearizable. In the next few paragraphs, it will be shown that conditions (5.3.9) and (5.3.10) hold only if the span angle of robot's body satisfies the condition: $\phi=\phi_{o} p t$.

First we re-define the control input $u$ in (5.3.1) as

$$
\hat{u}=f_{4}+g_{4} u
$$

such that (5.3.1) can be written as

$$
\dot{\mathbf{x}}=f(\mathbf{x})+g\left(x_{2}\right) \hat{u}
$$


where

$$
\begin{aligned}
f(x) & =\left[\begin{array}{c}
x_{3} \\
x_{4} \\
\frac{-m_{1} \sin \left(x_{2}\right) x_{4}^{2}+m_{5} \sin \left(x_{1}\right)-m_{6} \sin \left(x_{1}+x_{2}\right)-2 x_{3} m_{1} \sin \left(x_{2}\right) x_{4}-x_{3} f_{r 1}-T_{c 1}}{m_{2}-2 m_{1} \cos \left(x_{2}\right)} \\
0
\end{array}\right] \\
g\left(x_{2}\right) & =\left[\begin{array}{c}
0 \\
0 \\
\frac{m_{3}-m_{1} \cos \left(x_{2}\right)}{-m_{2}+2 m_{1} \cos \left(x_{2}\right)} \\
1
\end{array}\right]
\end{aligned}
$$

with state vector $\mathbf{x}$ defined as $x_{1}=q_{1}, x_{2}=q_{2}, x_{3}=\dot{q}_{1}$ and $x_{4}=\dot{q}_{2}$. The control matrix $M$ for the simplified system (5.3.12) can be computed using (5.3.8). A non-zero determinant $\operatorname{det}(M) \neq 0$ indicates that the control matrix is full rank. Hence, the simplified system (5.3.12) is controllable. On the other hand, the simplified system is not involutive for the Lie expression $\left[w_{2}, w_{3}\right] \neq 0$ is true, suggesting that (5.3.12) is not input-state linearizable.

If we, however, set the body angle $\phi$ to $\phi_{\text {opt }}$ forcing $m_{1}=m_{3}$ between the constants and assume relatively small values for the states $x_{2}$ and $x_{4}$ (thus, allowing higher order terms in $x_{2}$ and $x_{4}$ to be ignored), $g(3)$ in (5.3.12) reduces to zero, further simplifying the dynamics to

$$
\dot{x}=\tilde{f}(\mathbf{x})+\tilde{\mathbf{g}} \hat{u}
$$

where

$$
\begin{aligned}
\tilde{f}(x) & =\left[\begin{array}{cc}
x_{3} \\
x_{4} \\
\frac{-T_{c 1}+m_{5} \sin \left(x_{1}\right)-m_{6}\left(\sin \left(x_{1}\right)+x_{2} \cos \left(x_{1}\right)\right)-x_{3} f_{r 1}}{m_{2}-2 m_{1}} & m_{2} \neq 2 m_{1} \\
& 0
\end{array}\right] \\
\tilde{g} & =\left[\begin{array}{llll}
0 & 0 & 0 & 1
\end{array}\right]^{T}
\end{aligned}
$$


with controllability matrix, $\tilde{M}$ given by

$$
\tilde{M}=\left[\begin{array}{cccc}
0 & 0 & 0 & m_{22} \cos \left(x_{1}\right) \\
0 & -1 & 0 & 0 \\
0 & 0 & -m_{22} \cos \left(x_{1}\right) & m_{22}\left(\sin \left(x_{1}\right) x_{3}-\frac{f_{r 1} \cos \left(q_{1}\right)}{m_{2}-2 m_{3}}\right) \\
1 & 0 & 0 & 0
\end{array}\right]
$$

where $m_{22}=\frac{m_{6}}{m_{2}-2 m_{3}}$ is a constant. The determinant of $\tilde{M}$ is given by $m_{22}^{2} \cos \left(x_{1}\right)^{2}$, which is never zero unless $x_{1}= \pm 90^{\circ}$. It can also be shown that the Lie expressions

$$
[\tilde{M}(:, 1), \tilde{M}(:, 2)]=[\tilde{M}(:, 1), \tilde{M}(:, 3)]=[\tilde{M}(:, 2), \tilde{M}(:, 3)]=0
$$

In other words, if $x_{1} \neq 90^{\circ}$, conditions (5.3.9) and (5.3.10) hold true, rendering the reduced system 5.3.15 feedback or input-state linearizable.

After verifying the conditions for input-state linearizability, the next step is to find the coordinate transformation such that the simplified plant dynamics (5.3.15) is transformed into an equivalent linear time-invariant dynamics of the form $\dot{\mathbf{z}}=\mathbf{A z}+\mathbf{B} v$. Denoting the new coordinate as $z=T(\mathbf{x}), z(0)=0$, the first transformation, $z_{1}$, is usually obtained by taking the simplest solution to the partial differential equations

$$
\frac{\partial T_{1}}{\partial x_{2}}=0, \quad \frac{\partial T_{1}}{\partial x_{3}}=0 \quad \frac{\partial T_{1}}{\partial x_{4}}=0 \quad \frac{\partial T_{1}}{\partial x_{1}} \neq 0 .
$$

Choosing

$$
z_{1}=T_{1}=x_{1}
$$

the remaining three coordinate $z_{2}, z_{3}$, and $z_{4}$ are obtained from the relation $[57,510]$

$$
z_{i}=T_{i}=<d T_{i-1}, f>=\sum_{j=1}^{4} \frac{\partial T_{i-1}}{\partial x_{i}} f_{i} .
$$


After performing the math, we obtain the following coordinate transformation

$$
\left[\begin{array}{c}
z_{1} \\
z_{2} \\
z_{3} \\
z_{4}
\end{array}\right]=\left[\begin{array}{c}
T_{1} \\
T_{2} \\
T_{3} \\
T_{4}
\end{array}\right]=\left[\begin{array}{c}
x_{1} \\
x_{3} \\
\frac{m_{6}\left(\cos \left(x_{1}\right)-x_{2} \sin \left(x_{1}\right)\right)-m_{5} \cos \left(x_{1}\right)}{-m_{2}+2 m_{3}} x_{3}+m_{6} \frac{\cos \left(x_{1}\right)}{-m_{2}+2 m_{3}} x_{4}+\ldots \\
\frac{f_{r 1}\left(T_{c 1}+m_{6}\left(\sin \left(x_{1}\right)+x_{2} \cos \left(x_{1}\right)\right)+x_{3} f_{r 1}-m_{5} \sin \left(x_{1}\right)\right)}{\left(-m_{2}+2 m_{3}\right)^{2}}
\end{array}\right]
$$

and feedback linearizing control input $\hat{u}$ given by $[57,510]$

$$
\hat{u}=\frac{v}{\left\langle d T_{4}, g\right\rangle}-\frac{\left\langle d T_{4}, f\right\rangle}{\left\langle d T_{4}, g\right\rangle}=\xi(x) v+\varrho(x)
$$

where

$$
\begin{aligned}
& \xi(x)= \frac{2 m_{3}-m_{2}}{m_{6} c \cos \left(x_{1}\right)} \\
& \varrho(x)=\left[\frac{\left(m_{6}\left(-\sin \left(x_{1}\right)-x_{2} \cos \left(x_{1}\right)\right)+m 5 \sin \left(x_{1}\right)\right) x_{3}^{2}-m_{6} \sin \left(x_{1}\right) x_{3} x_{4}}{-m_{2}+2 m_{3}}+\ldots\right. \\
& \frac{f_{r 1} x_{3}\left(m_{6}\left(\cos \left(x_{1}\right)-x_{2} \sin \left(x_{1}\right)\right)-m_{5} \cos \left(x_{1}\right)\right)}{\left(-m_{2}+2 m_{3}\right)^{2}}+\ldots \\
& \quad \frac{-m_{6} x_{3} x_{4} \sin \left(x_{1}\right)}{-m_{2}+2 m_{3}}+\frac{f_{r 1} x_{4} m_{6} \cos \left(x_{1}\right)}{\left(-m_{2}+2 m_{3}\right)^{2}}+\ldots \\
&\left(\frac{m_{6}\left(\cos \left(x_{1}\right)-x_{2} \sin \left(x_{1}\right)\right)-m_{5} \cos \left(x_{1}\right)}{-m_{2}+2 m_{3}}+\frac{f_{r 1}^{2}}{\left(-m_{2}+2 m_{3}\right)^{2}}\right) \\
&\left.\quad\left(\frac{T_{c 1}+m_{6}\left(\sin \left(x_{1}\right)+x_{2} \cos \left(x_{1}\right)\right)+x_{3} f_{r 1}-m_{5} \sin \left(x_{1}\right)}{-m_{2}+2 m_{3}}\right)\right] \frac{2 m_{3}-m_{2}}{m_{6} \cos \left(x_{1}\right)}
\end{aligned}
$$

Resulting from the input and state transformation is the following set of linear equations

$$
\begin{aligned}
& \dot{z}_{1}=z_{2} \\
& \dot{z}_{2}=z_{3} \\
& \dot{z}_{3}=z_{4} \\
& \dot{z}_{4}=v .
\end{aligned}
$$

To force integral action into the control law for the elimination of steady-state error, one can augment the state transformation $Z$ with an integral of $x_{1}$ such that the augmented 
system in canonical form will be given by

$$
\dot{\mathbf{z}}=\mathbf{A z}+\mathbf{B} v
$$

where

$$
\mathbf{z}=\left[\begin{array}{c}
\int z_{1} \\
z_{1} \\
z_{2} \\
z_{3} \\
z_{4}
\end{array}\right] \quad A=\left[\begin{array}{lllll}
0 & 0 & 1 & 0 & 0 \\
0 & 0 & 0 & 1 & 0 \\
0 & 0 & 0 & 0 & 1 \\
0 & 0 & 0 & 0 & 0
\end{array}\right] \quad B=\left[\begin{array}{l}
0 \\
0 \\
0 \\
0 \\
1
\end{array}\right]
$$

For stability, $v$ is chosen as

$$
v=-K_{E F L} \mathbf{z}
$$

such that the matrix $\mathbf{A}-\mathbf{B K}_{\mathbf{E F L}}$ is Hurwitz $[57,238]$. The overall nonlinear control for the original system, (2.3.10), is obtained using (5.3.26), (5.3.22), and (5.3.11).

\subsection{Simulation}

In order to obtain a fair comparison, the control gains for both the LQR and Feedback Linearizing controllers are based on a common set of closed loop poles. At this stage of the experimentation, the focus is simply to verify the ability of this control solution to stabilize the robot. Integral action, is therefore, omitted at this stage from the Feedback Linearizing strategy. Closed loop gains of the LQR without integral action, but close in location to LQR with integral action used in the previous simulation, is initially considered.

Using the state and control matrices

$$
\mathbf{Q}=\left[\begin{array}{cccc}
300 & 0 & 0 & 0 \\
0 & 200 & 0 & 0 \\
0 & 0 & 1 & 0 \\
0 & 0 & 0 & 1
\end{array}\right] \quad R=60
$$


for the LQR without integral action results in the following controller gains:

$$
\mathbf{K}_{L Q R}=\left[\begin{array}{llll}
-100.7650 & 13.9073 & -24.8712 & -0.0324
\end{array}\right]
$$

As in the previous chapter, these are the smallest LQR controller gains that will stabilize the simulated system. The continuous-time closed loop poles corresponding to the above controller gains are $-41.2144,-4.0215,-3.1210$, and -1.0368 . Discrete closed loop poles are not used to synthesize the Feedback Linearizing controller gains for the sampling effect of the transformation given in (5.3.23) is not known. Using the Ackermann method [53, 245], in conjunction with the above closed loop poles, the nonlinear control gains $K_{E F L}$ without integral action are given as:

$$
\mathbf{K}_{E F L}=\left[\begin{array}{llll}
536.2861 & 835.4767 & 357.0555 & 49.3936
\end{array}\right]
$$

The efficacy of the controller in rejecting four types of disturbances, introduced in Chapter 4 , will be considered. The first type of disturbance is a constant voltage applied at the servo amplifier input. The second type of disturbance considered is a periodic voltage applied to the input of the servo amplifier. A disturbance comprising of two opposing pulses, $1 \mathrm{sec}$ in width, spaced $20 \mathrm{sec}$ apart is also considered. Each pulse has an absolute magnitude of $1 \mathrm{~V}$. These disturbances are applied with all system states initially set to zero. Finally, the system states are subjected to non-zero initial values and the controller behavior is monitored. To add some degree of reality, nonlinearities such as deadband, white noise in the control input, and a ZOH element are all included in the Simulink model. As in Cshapter 4 , angular velocities estimates are obtained by differentiating the positional vectors and applying the result through a first order low pass filter with a cutoff frequency of $2 \mathrm{~Hz}$. 


\subsubsection{Constant Voltage Disturbance}

The constant disturbance tested comprises a constant voltage signal applied persistently to the servo amplifier driving the robot's DC motor. This disturbance, $\mathrm{C}$, enters the system through the same channel as the control input i.e.

$$
V_{a}=u+C
$$

where $V_{a}$ is the actual voltage applied to the servo amplifier, $u$ is the computed control voltage, and $\mathrm{C}$ is the constant disturbance. A value of $\mathrm{C}=0.1 \mathrm{~V}$ was used in the simulation. It can be observed from Figure 5.1 that the constant voltage disturbance forces the FL controller to balance the robot about an equilibrium other than the desired. One can also observe that the closed loop behavior of the system under FL and LQR control is similar. Another observation is that the FL controller is a little more sluggish than the LQR control.

\subsubsection{Periodic Voltage Disturbance}

The periodic disturbance tested comprises a $1 \mathrm{~Hz}$ sinusoidal voltage signal with an amplitude of $2 \mathrm{~V}$. As before, this disturbance is persistently applied through the same channel as the control input i.e.

$$
V_{a}=u+2 \sin (2 \pi t)
$$

where $V_{a}$ is the actual voltage applied to the servo amplifier and $u$ is the computed control voltage. The simulation result presented in Figure 5.2, reveals a balanced system, even though the disturbance is hardly attenuated. For such small disturbances, one can hardly distinguish the response of the system under LQR and FL control. 


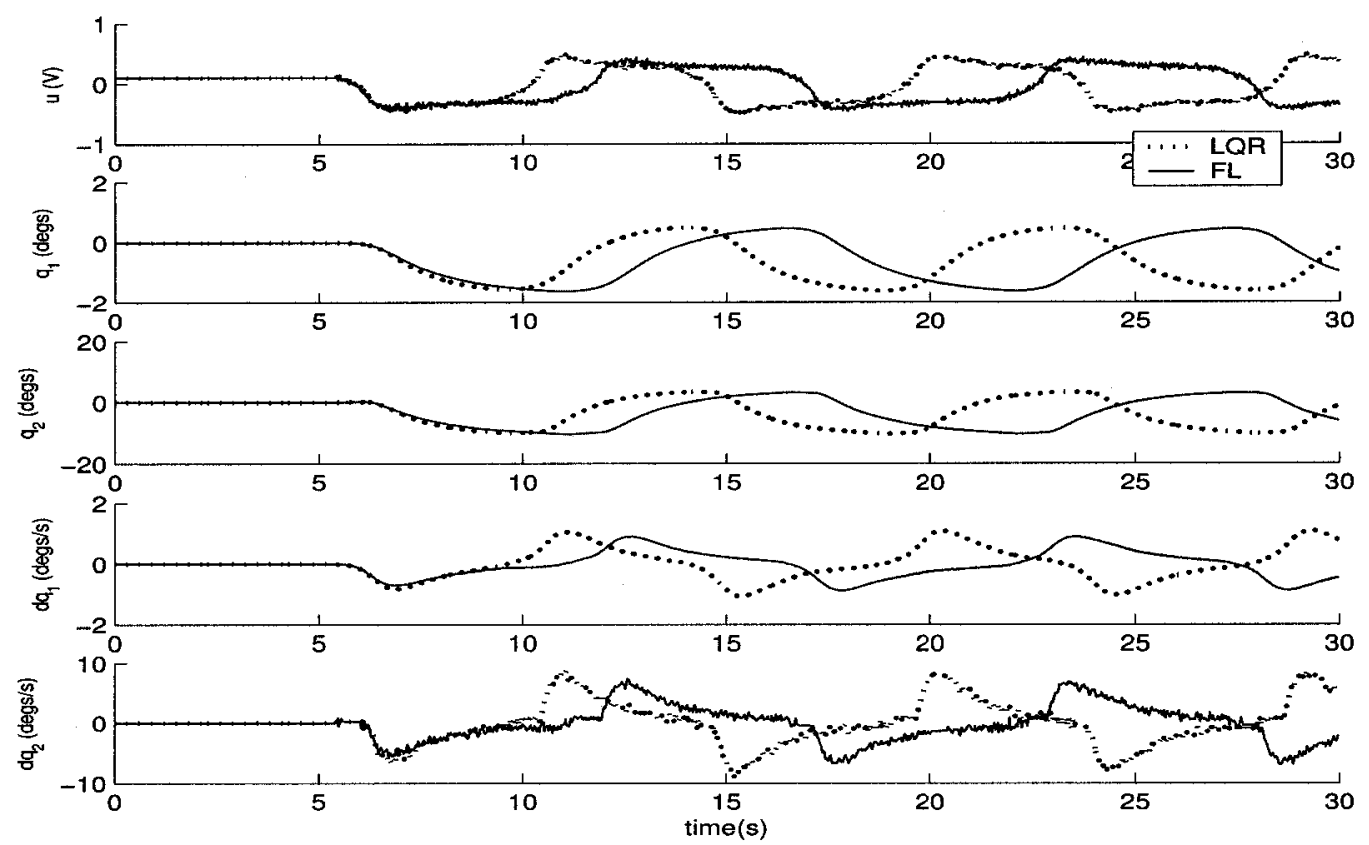

Figure 5.1: Simulation Response of FL vs. LQR Control in Response to Constant Disturbance

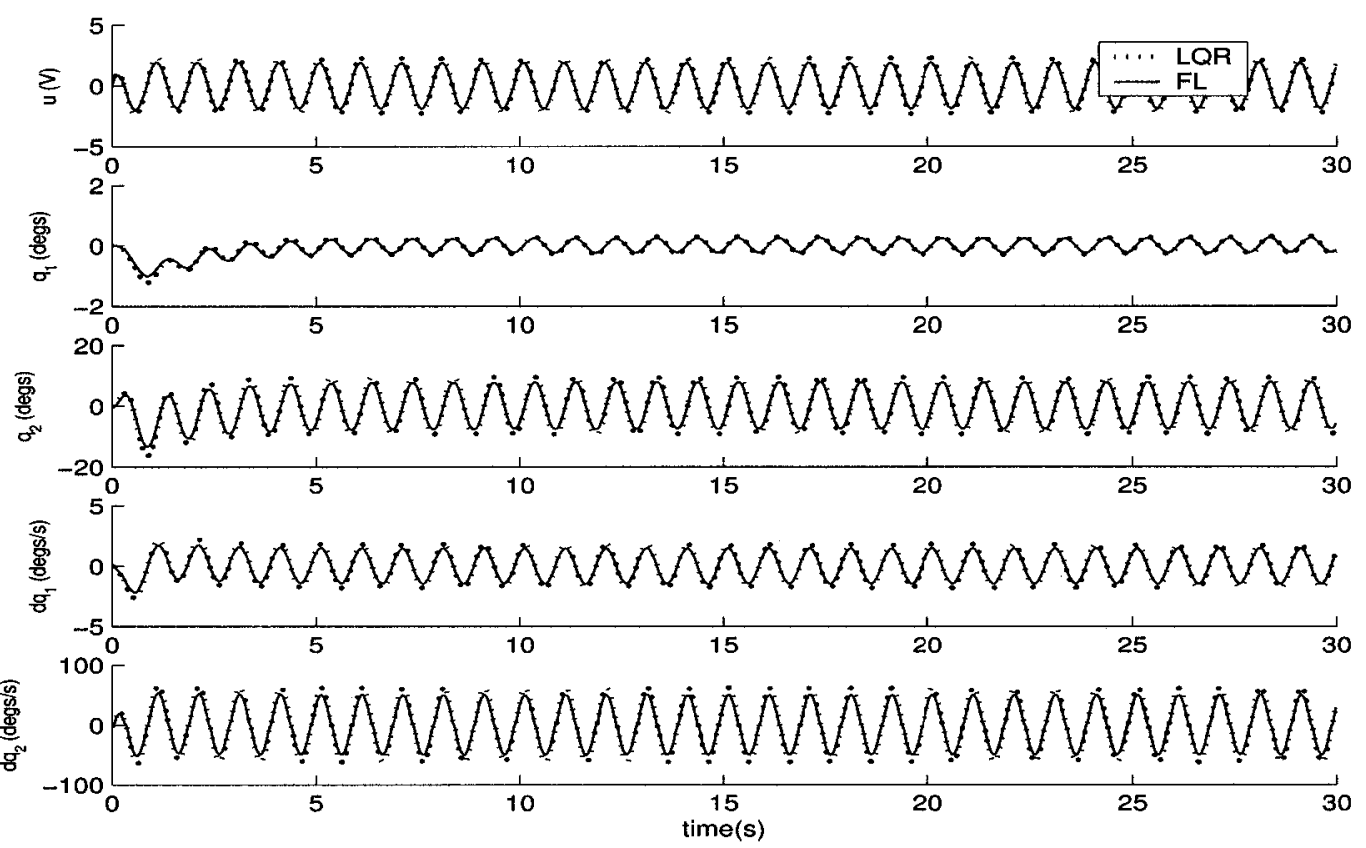

Figure 5.2: Simulation Result of FL vs. LQR Control in Response to a Sinusoidal Disturbance 


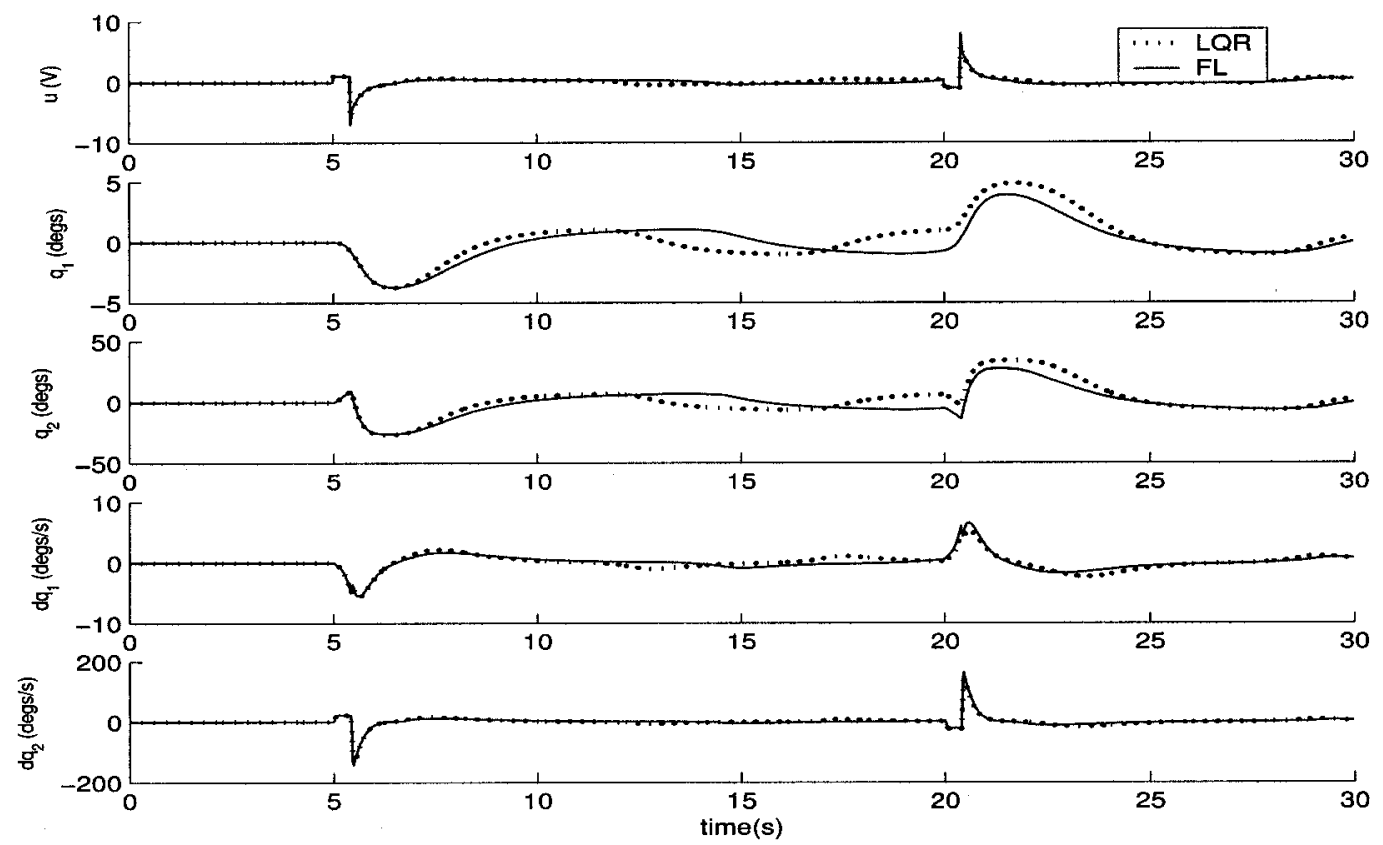

Figure 5.3: Simulation Result of FL vs. LQR in Response to 2 Pulses

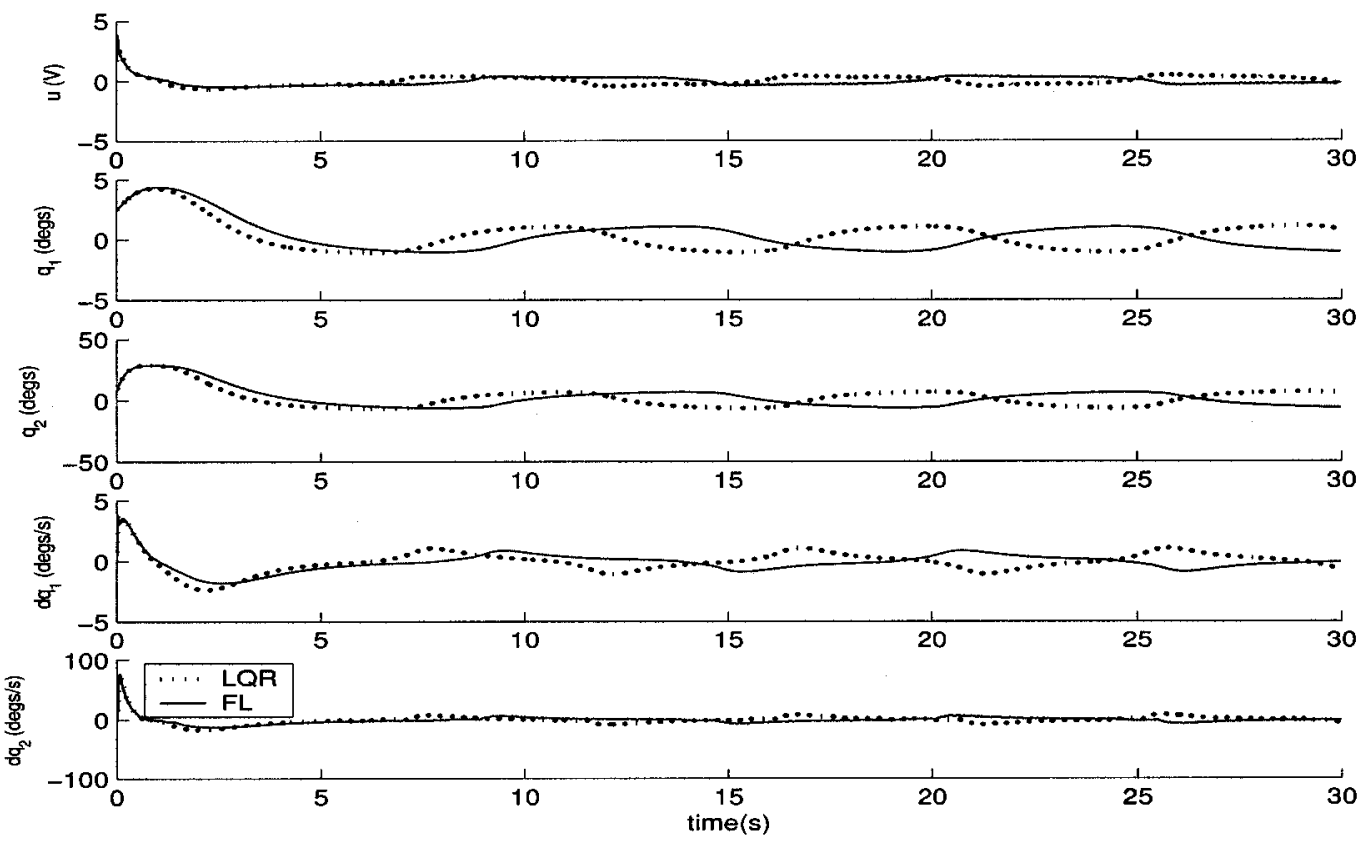

Figure 5.4: Simulation Result of FL vs. LQR Control in response to Non-Zero Initial Conditions 


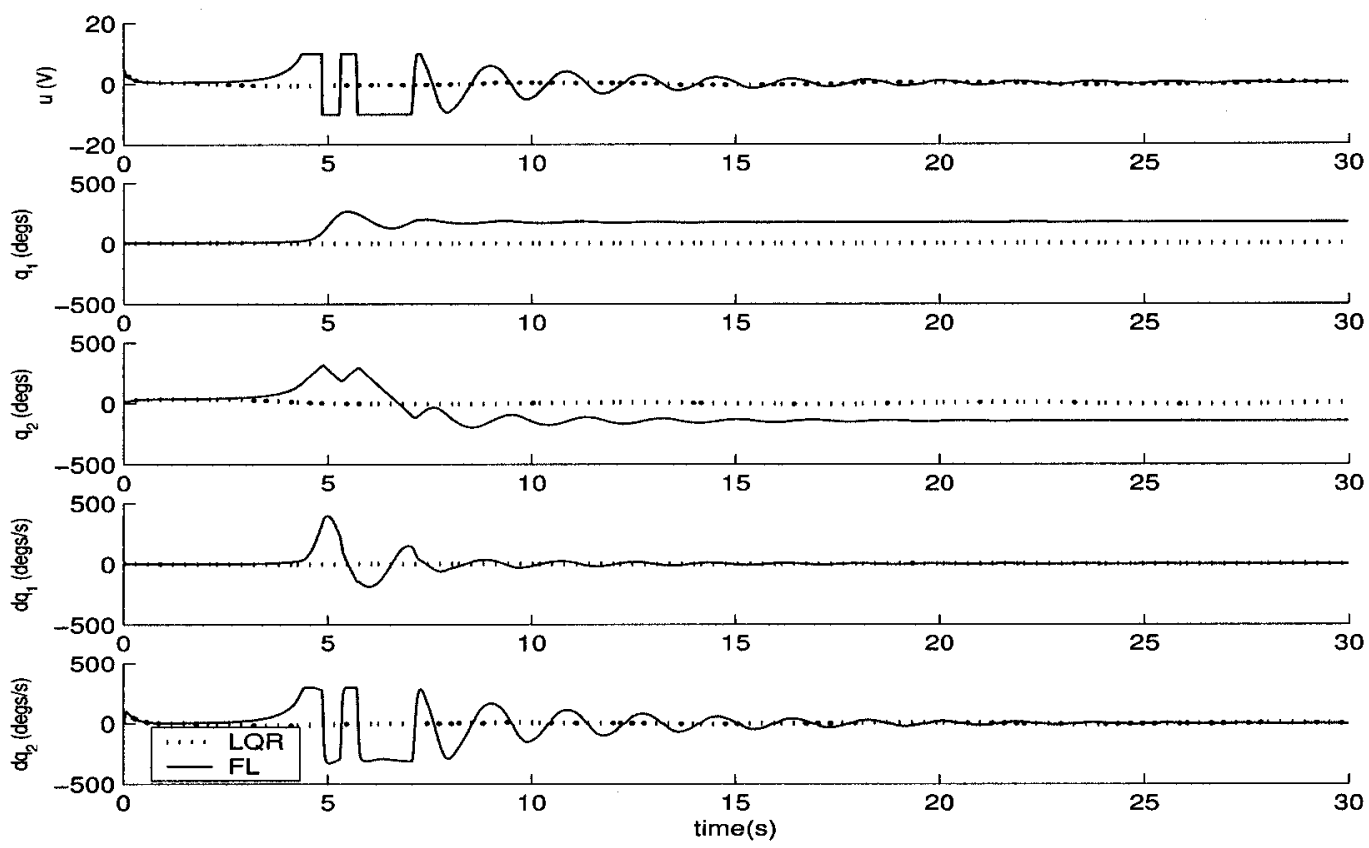

Figure 5.5: Simulation Result of FL vs. LQR Control in response to Non-Zero Initial Conditions

\subsubsection{Pulsed Voltage Disturbance}

A disturbance comprising two opposing voltage pulses, $1 \mathrm{sec}$ wide, $20 \mathrm{sec}$ apart, each with an absolute magnitude of $1 \mathrm{~V}$, is also considered. In contrast to the two previous cases, this disturbance is momentarily applied with the system in open loop before the nonlinear FL is applied. Figure 5.4.3 reveals an overall satisfactory response of the nonlinear FL controller. One also can observe the similarities in the response between the LQR and FL controller.

\subsubsection{Initial Displacement from Equilibrium}

The state variables are initially set as follows: $q_{1}=2.5^{\circ}, q_{2}=10^{\circ}, \dot{q}_{1}=4^{\circ} / \mathrm{sec}$ and $\dot{q}_{2}=-5^{\circ} /$ sec. The arbitrary initial velocities are chosen such that the first link is moving further away from the equilibrium position (counterclockwise), while the second link is moving clockwise in relation to the first link. As depicted in Figure 5.4, the FL controller 
is able to stabilize the system for this set of initial conditions. Again, the response of the nonlinear FL controller is very similar to that of the LQR contoller. In an effort to determine

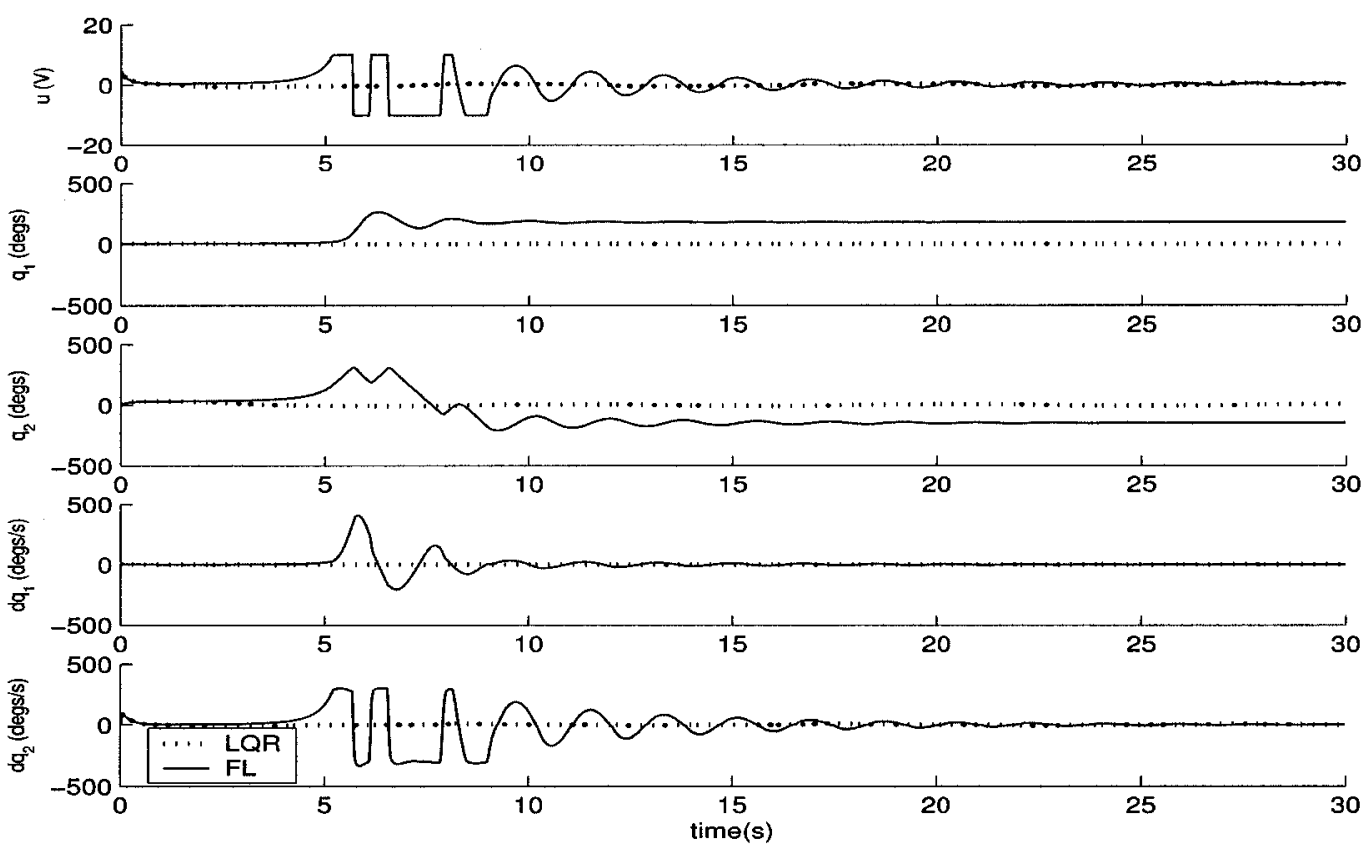

Figure 5.6: Simulation Result of FL vs. LQR Control in response to Non-Zero Initial Conditions

which control system offers the greater region of attraction, a simulation is carried out with the state variables $q_{1}$ and $q_{2}$ systematically increased and both controllers run in unison. The response of the FL and LQR controllers always follow the pattern already observed: The LQR control offers a quicker response to system disturbances than the FL controller. In terms of the region of attraction, the LQR offers a significant advantage, as illustrated in Figure 5.5. In this case, the initial state disturbances are set to $q_{1}=3^{o}, q_{2}=10^{\circ}$, $\dot{q}_{1}=4^{\circ} /$ sec, and $\dot{q}_{2}=-5^{\circ} /$ sec. While the LQR is still able to stabilize the system, the FL control fails.

A sensitivity analysis was also performed in Section 3.6 on the estimated model parameters. It was observed that uncertainty in the moment of inertia of link 2 and the efficiency 
factor, $l_{c 2}$ and $\eta$ respectively, largely account for modelling discrepancy. To test for controller robustness, a $+40 \%$ increase in $l_{c 2}$ and $\eta$ is applied to the model subjected to initial conditions $q_{1}=2.5^{\circ}, q_{2}=10^{\circ}, \dot{q}_{1}=4^{\circ} / \mathrm{sec}$, and $\dot{q}_{2}=-5^{\circ} / \mathrm{sec}$. Both controllers are still able to stabilize the system. Interestingly enough, when a $+40 \%$ uncertainty is introduced in $I_{L 1}$, the moment of inertia of the leg, the FL controller fails to stabilize the system for the aforementioned set of initial conditions, while the LQR is still able to stabilize the system.

\subsection{Experimental Results}

Figure 5.7 below depicts the response of the FL control in the real system subjected to 2 second interval unit pulses with opposing polarity, spaced 30 seconds apart. It can be observed that the nonlinear control law is able to stabilize the real system, even though the actual body angle $\phi=40^{\circ}$ is not equal to $30^{\circ} \leq \phi_{\text {opt }} \leq 40^{\circ}$. Given that numerous

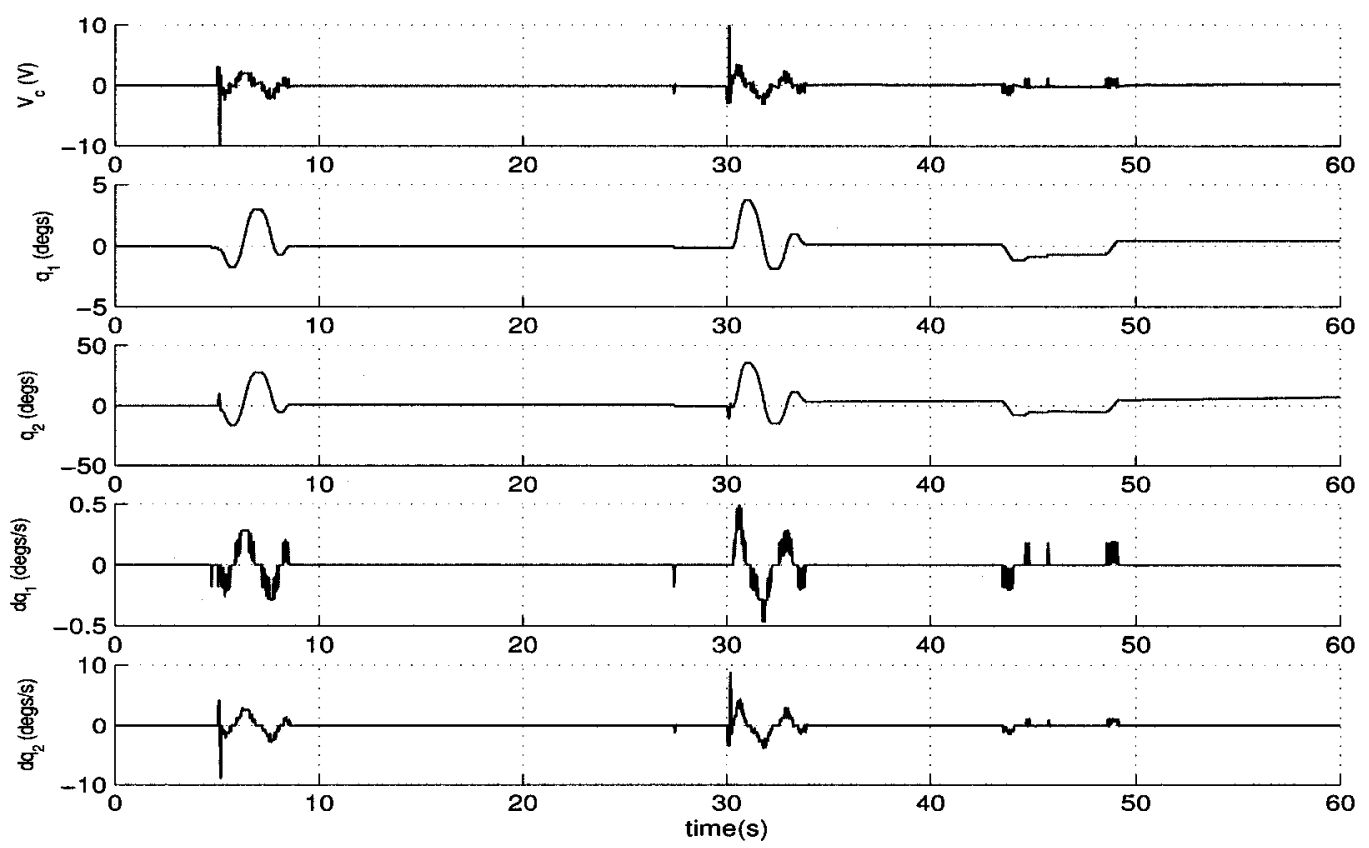

Figure 5.7: Experimental Result of FL control in response to 2 pulses 
simulation results do not reveal any significant advantage of the FL over the LQR design, a limited experimental data was collected with this control in action.

\subsection{Conclusion}

In this chapter, a nonlinear Feedback Linearizing (FL) controller was synthesized to maintain balance in a 2 DOF one-legged hopping robot while offering a large region of attraction. Even though the synthesized nonlinear controller was able to stabilize the robot for small disturbances, its performance did leave a lot to be desired, especially when compared against that of the LQR. Specifically, the LQR offered a larger region of attraction, had a quicker response to system disturbances, and was less sensitive to parametric and modelling uncertainty when compared against the FL controller. The synthesized nonlinear controller, therefore, did not meet the objectives for which it was original designed.

Feedback Linearization is a powerful control strategy, nonetheless, when an accurate mathematical model of a system is available and the system dynamics is feedback linearizable [27, 207]. Otherwise, as it has been observed, the control does not guarantee the performance inherent in other nonlinear control strategies. When using the robotic system parameters computed using mathematical relationships such that the moment of inertia of link 2 does not violate one of the conditions required for feedback linearizaton, as mentioned earlier in this chapter, the nonlinear FL controller outperforms the LQR. Specifically, for mathematically computed system parameters, the FL controller is able to stabilize the system subjected to initial displacements of $q_{1}$ as large as $25^{\circ}$, while the region of attraction for the LQR is restricted to $5^{\circ}$.

The shortcomings portrayed by the FL control can, therefore, be mapped to assumptions that were made for the feedback linearization conditions to be satisfied. In particular, the 
discrepancy between estimated and mathematically computed system parameters, which violates a condition required for feedback linearization, is a major impediment. To achieve the control objectives of large operation range and robustness in the face of parametric or modelling uncertainty, FL is, therefore, not a suitable candidate for this application. Other nonlinear control strategies must be sought. 


\section{Chapter 6}

\section{Conclusion and Future Works}

The overall goal of this project was to design a power conserving mechanism that can be used to maintain postural stability in a hopping robot in the presence of modelling and parametric uncertainty. The mechanical balancing mechanism proposed is a downward swinging concave body mounted on a non-compliant piece of wood serving as the leg of a monopod during stance.

The body span angle, referred to as $\phi$, was chosen as a design parameter as it was observed that location of the open loop transfer function zeros can be altered simply by changing $\phi$. Furthermore, it was shown that transfer function zeros disappear for a specific body angle, referred to as $\phi_{\text {opt }}$. At this body configuration, the system was deemed to be minimum phase, conditions for feedback linearization of the nonlinear model were satisfied, and a reduction in minimal mechanical power required for stability during stance was observed.

In Chapter 3, a customized nonlinear gray-box system identification procedure was successfully used to estimate certain parameters in the dynamic model of the one-legged robotic prototype. The identification routine was run for several values of $\phi$ and a locus of the transfer function zeros was used to estimate the optimal angle at which the system has 
no transfer function zeros at all. The optimal angle was found to lie within the range of $30^{\circ}$ to $40^{\circ}$. A sensitivity analysis was also performed on the estimated model parameters. It was observed that uncertainty in $l_{c 2}$ and $\eta$ largely account for modelling discrepancy.

In Chapter 4, a LQR with Integral action was designed to balance the robot about its unstable equilibrium. This scenario mimics balance of a one legged hopping robot during stance. Simulation results demonstrated the effectiveness with which the LQR rejected different types of system disturbances. Numerous experimental results, with the system subjected to a pulsed disturbance, further confirmed that the controller will consistently reject disturbances that fall within a narrow operational range. Given the absence of a set of performance specifications, however, the system gains selected were very conservative.

Simulation results given in Chapter 4 also confirmed a relationship between the body angle $\phi$ and the control effort required to maintain system stability. Although the relationship has yet to be clearly specified, these preliminary findings provide enough evidence towards the claim in power conservation through a careful selection of $\phi$. Nonetheless, an experimental verification of the effect on control effort for changes in $\phi$ needs to be performed to substantiate this claim.

A fundamental limitation of the LQR is its limited operation range given that the control system is based on a model linearized about the desired equilibrium. In actual implementation, it would be desirable for the control system to be able to withstand larger operational ranges even in the presence of modelling uncertainty. In Chapter 5, a nonlinear control strategy was synthesized in an attempt to increase the operation range of the robot control system.

Even though the synthesized nonlinear controller was able to stabilize the robot for small disturbances, its performance was at least satisfactory especially when compared against that of the LQR. Specifically, the LQR offered a larger region of attraction, had a 
quicker response to system disturbances and was less sensitive to parametric and modelling uncertainty when compared against the FL controller. The synthesized nonlinear controller, therefore, did not meet the objectives for which it was originally designed.

The shortcomings portrayed by the FL control were attributed to assumptions that were made for the feedback linearization conditions to be satisfied. In particular, the discrepancy between estimated and mathematically computed system parameters, which violated a condition required for feedback linearization,s was found to be the major contributor to the lackluster performance of the nonlinear controller. To achieve the control objectives of large operation range and robustness in the face of parametric or modelling uncertainty, it was concluded that other nonlinear control strategies should be sought.

\subsection{Problems Encountered and Future Works}

Numerous challenges were encountered in this project. From mathematical modelling to system identification and control, numerous questions have remained unanswered. Some of the unexplained phenomena and problems encountered are listed below. A suggested recommendation for further works follows each encountered problem:

Even though the identified system parameters result in a close match between the real and simulated systems, the parameters by themselves, or when compared to the mathematically computed counterpart, differ significantly. For the purposes of control, however, the estimated parameters suffice. Nevertheless, it must be determined if it is sufficient to attribute the significant difference between the estimated and computed parameters to a discrepancy between the real and mathematical model.

Selection of a sampling rate for the closed loop system also did not prove to be a straight forward task. Even though the system was successfully stabilized using a sampling rate based on the desired closed loop bandwidth, it was observed in passing that the selected 
sampling rate may not have been optimal. This can be attributed to the fact that selection of a closed loop bandwidth was not based on performance objectives but solely on the excitation of un-modelled dynamics. For better performance, it may be necessary to specify performance objectives such that a desired closed loop bandwidth, and subsequently an optimal sampling rate, can be obtained.

Sensor positions proved to be a major contributor to the overall stability problem of the system. After breaking numerous motors due to vibrations induced from flexibilities in the gear train when an encoder was mounted on the body itself, a decision was made to use the encoder attached to the motor instead as well as resort to a chain and sprocket assembly. These changes reduced system vibrations to a manageable level. However, unless the transmission is taut, there occurs an error between the actual body rotational displacement and that computed by the motor encoder. This error propagates through the system, consequently reducing system performance.

\subsection{Additional Recommendations}

Overall, the LQR controller performed satisfactorily, providing some degree of robustness in the face of parametric uncertainty. However, the LQR formulation is not a robust control strategy. For wider range operation and robustness in the presence of modelling and parametric uncertainty, a robust control scheme must be employed.

After stability, performance is key. Future work on this project should begin with a definition of an ambitious set of performance objectives.

Effort should also be put into the selection of an optimal sampling frequency. The sampling rate selected should be just fast enough as higher sampling rates place more computation burden on the processor, thus, increasing the power consumption of the system. 
Also, the overall power consumption of the system needs to be tested in comparison with a predefined benchmark. The proposed balancing mechanism, in theory, reduces the mechanical power consumption of such robotic structures. Experimental results or data will have to be collected to further validate this claim. 


\section{Bibliography}

[1] G. Stein. Respect the unstable. IEEE Control Systems Magazine, Aug 2003. 0272-1708.

[2] K. Matsouka. A model of repetitive hopping movements in man. Proc. Fifth World Congress. Theory of Machines and Mechanisms, 1979.

[3] M. H. Raibert. Legged Robots That Balance. MIT Press, Cambridge, 1997.

[4] R. R Playter. Passive Dynamics in the Control of Gymnastic Maneouvers. PhD thesis, M. I. T., Cambridge, MA, 1994.

[5] M. W. Spong and L. Praly. Control of underactuated mechanical systems using switching and saturation. Proc. of the Block Island Workshop on Control Using Logic Based Switching, 1996.

[6] M. W. Spong and L. Praly. Energy based control of a class of underactuated mechanical systems. IFAC World Congress, July, 1996.

[7] M. W. Spong and D. J. Block. The pendubot: A mechatronic system for control research and education. Proc. of the 34th IEE Conference on Decision and Control, pages 555-556, Dec. 1995. New Orleans.

[8] P. Hsu. Dynamics and control design project offers taste of real world. IEEE Control Systems Magazine, pages 31-38, Jun. 1992.

[9] M. W. Spong. The swing-up control problem of the acrobot. IEEE Systems Magazine, 1995. 15:49-55. 
[10] M. W. Spong and D. J. Block. The pendubot: A mechatronic system for control research and education. Proc. of the 34 th IEEE Conference on Decision and Control, Dec. 1995. p. 555-556, New Orleans.

[11] L. Qui and E. Davison. Performance limitations of non-minimum phase systems in the servomechanism problem. Automatica, 29(2):337-349, 1993.

[12] NASA Jet Propulsion Laboratory. Mars exploration rover mission. marsrovers.jpl.nasa.gov.

[13] R. Ringrose. Self Stabilizing Running. PhD thesis, M. I. T., Cambridge, MA, 1997.

[14] G. Zeglin and H. Brown. Control of a bow leg hopping robot. Proceedings of the IEEE International Conference on Robotics and Automation, 1998. Leuven, Belgium.

[15] K. V. Papantoniou. Control architecture for an electrical actively balanced multi-leg robot based on experiments with a planar one leg machine. IFAC Robot Control, 1991. Vienna, Austria.

[16] P. Gregorio H. Rad and M. Buehler. Design, modeling and control of a hopping robot. IEEE/RSJ Conf. Intelligent Robots and Systems, Jul. 1993. Yokohama, Japan.

[17] R. Dummer and M. Berkemeier. Low-energy control of a one-legged robot with 2 degrees of freedom. Proc. of the IEEE Int. Conference on Robotics and Automation, 2000. San Francisco, CA.

[18] M. Ahmadi and M. Beuhler. Stable control of a simulated one-legged hopping robot with hip and leg compliance. IEEE Transactions on Robotics and Automation, 13(1), 1997.

[19] T. McGeer. Passive dynamic walking. International Journal of Robotics Research, $9(2): 62-82,1995$.

[20] D. E. Koditschek and Bhler. Analysis of a simplified hopping robot. International Journal of Robotics Research, 10(6):587-605, 1991. 
[21] Burdick J. W. Vakakis, A. F. and T. Caughey. An interesting strange attractor in the dynamics of a hopping robot. International Journal of Robotics Research, 10(6):606$618,1991$.

[22] J. B. Helferty J. J., Collins and M. Kam. Adaptive control of a legged robot using an artificial neural network. Proceedings of the American Control Conferennce, pages $165-168,1989$.

[23] B. Surgenor M. Mehrandezh and S. Dean. Jumping height control of an electrically actuated hopping robot: Modeling and simulation. IEEE Proc. of the 34th Conference on Decision and Control, 1995. New Orlenas, LA.

[24] W. J. Schwind and D. E. Koditschek. Control of forward velocity for a simplified planar hopping robot. Technical Report,EECS, Department, College of Engineering, 121994. Ann Arborm, MI.

[25] G. Becker S. Pannu and H. Kazerooni. Stability of a one legged robot using $\mu$-synthesis. IEEE International Conference on Robotics and Automation, 1995.

[26] H. Yoshinda M. Gokan, K. Yamafuji. Postural stability and motion control of a rope hopping robot. JSME International Journal, 37(4), 1994.

[27] J-J. E. Slotine. Applied Nonlinear Control. Prentice Hall, Englewood Cliffs, NJ, 1991.

[28] Sony Corporation. Sony dream robot, qrio. www.sony.net/SonyInfo/QRIO/.

[29] M. Heverly P. Fiorini, S. Hayati and J. Gensler. A hopping robot for planetary exploration. Int. Proc. of IEEE Aerospace Conference, March 1999. Snowmass, CO.

[30] L. Sciavicco and B. Siciliano. Modelling and Control of Robotic Manipulators.

[31] E. Papadopoulos and G. Chasparis. Analysis and model-based control of servomechanisms with friction. Proc of the International Conference on Intelligent Robotics and Systems, 3(30):2109-2114, Sept. 2002.

[32] B. C. Kuo, Automatic Control Systems. Prentice Hall, Englewood Cliffs, NJ, 1995. 
[33] D. S. Bernstein. A student's guide to classical control. Aerospace Engineering Department, University of Michigan.

[34] J-J. E. Slotine. Applied Nonlinear Control. Prentice Hall, Englewood Cliffs, NJ, 1991. p. 258 .

[35] J. S. Freudenberg and D. P. Looze. Right half plane poles and zeros and design tradeoffs in feedback systems. IEEE Transaction on Automatic Control, AC-30(6), Jun. 1985.

[36] J-J. E. Slotine. Applied Nonlinear Control. Prentice Hall, Englewood Cliffs, NJ, 1991. p. 226,253 .

[37] J-J. E. Slotine. Applied Nonlinear Control. Prentice Hall, Englewood Cliffs, NJ, 1991. p227-228.

[38] H. W. Bode. Feedback Amplifier. Van Nostrand, 1945.

[39] B. A. Francis and G. Zames. On optimal sensitivity theory for siso feedback systems. IEEE Transaction on Automatic Control, AC-29, Jan. 1984.

[40] R. Middleton J. Freudenberg and A. Stefanopoulou. A survey of inherent design limitations. Proceedings of the American Control Conference, Jun. 2000.

[41] R. H. Middleton G. C. Goodwin, A. R. Woodyatt and J. Shim. Fundamental limitations due to $j \omega$ axis zeros in siso systems. Technical Report EE9723, Feb. 1998.

[42] J. J. Craig. Introduction to Robotics. Addison Wesley, 1989.

[43] O. Nelles. Nonlinear System Identification. Springer-Verlag, New York, 2001.

[44] Lennart Ljung. System Identification - Theory For the User. PTR Prentice Hall, 2 edition, 1999. Upper Saddle River, N.J.

[45] Advanced Motion Controls. www.a-m-c.com/.

[46] The Mathworks. Matlab and simulink for technical computing. www.mathworks.com.

[47] National Instruments. www.ni.com/. 
[48] W. Wang. Modeling scheme for longitudinal vehicle control. Proc of the 31th IEEE Conference of Decision and Control, 1:549-554, Dec. 1992. Tucson,AZ, USA.

[49] R. Pintelon and J. Schoukens. System Identification, A Frequency Domain Approach.

[50] L. Ljung. System Identification -Theory for the User.

[51] O. Nelles. Nonlinear System Identification. Springer-Verlag, 2001. Sec 7.2.2.

[52] B. A. Ogunnaike and W. H. Ray. Process Dynamics. Modeling and Control.

[53] J. D. Powell G. F. Franklin and M. L. Workman. Digital Control of Dynamic Systems.

[54] G. D. Sweriduk P. K. Menon and S. S. Vaddi. Nonlinear discrete time design methods for missile flight control systems. AIAA Guidance, Navigation and Control Systems, Aug 2004.

[55] I. J. Ha D. I. Kim and M. S. Ko. Control of an induction motor via feedback linearization with input-output decoupling. Intl. J. of Control, 51(4):863-886, Mar 1990.

[56] L. Guzella and A. M. Schmid. Feedback linearization of spark ignition engines with continuously variable transmission. IEEE Trans. on Control Systems Technology, 3, March 1995.

[57] H. K. Khalil. Nonlinear Systems. 


\section{Appendix A}

\section{Operating the 'Rotating Hip' Experimental Setup}

Follow the procedures and precautions outlined in this section, to setup the experiment and run the controller algorithm.

\section{Data Collection:}

1. The amplifier has been calibrated with a linear gain of $2.4 \mathrm{~V} / \mathrm{V}$. Ensure that this value has not drifted. Also, the amplifier is currently set to voltage mode.

2. Ensure that the voltage between + motor and - motor (amplifier output) is zero when the input reference pins ( + ref and -ref) is zero. Zero the input reference pins using the National Instruments MAX.

3. Do not adjust the potentiometer that is connected to one of the input pins of the amplifier. This potentiometer has been set to limit the maximum current in the amplifier to match $90 \%$ of the maximum motor current. To adjust this limit, please refer to the amplifier and motor manuals. They can be obtained online.

\section{Robot:}

1. Ensure that the chain is taught. You may have to loosen the body to retighten the chain. 


\begin{tabular}{|l|l|l|}
\hline & Encoder A & Encoder B \\
\hline Chan A & PFI8 & PFI3 \\
Chan B & DIO6 & DIO7 \\
\hline
\end{tabular}

2. Ensure that the set screws holding the motor shaft are tightened. Ensure that the set screws holding the body are also tightened.

3. Ensure that there is no play in the system.

4. Ensure that both encoders are securely connected to the BNC connector. The configuration of the encoder wires at the BNC connector should not be changed. The encoder wires should be connected as indicated in the table above.

CAUTION: I may have the above configuration reversed in this document, so please note the current configuration before disconnecting the wires.

5. Ensure that the encoder wires are plugged into the base of the encoders.

6. Ensure motor input terminals are connected to \pm motor terminals of servo amplifier.

\section{MATLAB:}

Two PC's in the lab contain both the system identification and controller simulation codes. One PC contains the system identification and simulation codes for it has the MATLAB Control System Toolbox. Another PC only runs the real time code for controller execution.

All system identification and controller MATLAB codes are in my possession and are available upon request.

Follow the steps listed below to run the controller:

1. Launch MALTAB 7.1; then open the supplied controller file rothip_realtime.m. Also open the supplied Simulink model rtwin_model.mdl.

2. The Simulink model contains all controllers under test, and each one is selectable by specifying either 1, 2, or 3 in the constant block labelled 'Control Switch.' The switches are: 
- $1=\mathrm{LQR}$

- $2=\mathrm{EFL}$

- $3=\mathrm{SMC}$

3. Before running the control, the file rothip_realtime.m must first be run to initialize the controller gains in the workspace. Simply run the MATLAB file.

(a) Within this file is defined a flag called 'intg'. Set this flag to 1 to add integral action into the control loop. Otherwise, leave the flag at zero. Its default value is zero.

(b) The m-script also contains another flag, 'test_switch.' This switch dictates the type disturbance applied to the system for controller testing. With this switch set to 1 , the system is disturbed using 2 bi-directional pulses spaced 20 seconds apart. This means that before the experiment is run, the robot has to be still in the upright position. In this mode, control starts only after the first disturbance pulse has been sent. There is no control action within the first 5 seconds. This is to allow the user to ensure that the system is still before the first pulse is applied. Setting the test switch to 2 allows the controller to kick in right away and no disturbance is applied.

(c) The m-script header fully explains all the constants in the file.

4. Once the m-script has been run, the user will have to invoke the build command within Simulink model rtwin_model.mdl to build the executable that will run on the RTWIN target.

5. Once the build is successful, as noted in the MATLAB command window, the user can then connect to the RTWIN target and start the controller.

6. The controller has been programmed to null its output 3 seconds before the end of the experiment. The controller has been programmed to run for 30 seconds.

7. The controller will also null its output once the body angle exceeds 70 degs to avoid the system from inflicting self-destruction. 
8. Please do not hesitate to contact me at Klaus.nji@gmail.com for further questions. 